\title{
Realising end invariants by limits of minimally parabolic, geometrically finite groups
}

\author{
KEN'ICHI OHSHIKA
}

\begin{abstract}
We shall show that for a given homeomorphism type and a set of end invariants (including the parabolic locus) with necessary topological conditions which a topologically tame Kleinian group with that homeomorphism type must satisfy, there is an algebraic limit of minimally parabolic, geometrically finite Kleinian groups which has exactly that homeomorphism type and the given end invariants. This shows that the Bers-Sullivan-Thurston density conjecture follows from Marden's conjecture proved by Agol and Calegari-Gabai combined with Thurston's uniformisation theorem and the ending lamination conjecture proved by Minsky, partially collaborating with Masur, Brock and Canary.
\end{abstract}

30F40, 57M50

\section{Introduction}

A Kleinian group is said to be geometrically finite when the corresponding hyperbolic 3-orbifold has a convex core which has finite volume. It was conjectured by Bers in [3] that every b-group, ie a Kleinian group with a unique simply connected invariant component in the domain of discontinuity, is an algebraic limit of quasi-Fuchsian groups. The conjecture was generalised by Sullivan and Thurston to the one saying that every finitely generated Kleinian group is an algebraic limit of quasiconformal deformations of a geometrically finite group. This generalised conjecture is called the Bers-Sullivan-Thurston density conjecture today. The original conjecture of Bers was solved by Bromberg [12] using deformations of cone manifolds and Minsky's solution of the ending lamination conjecture for hyperbolic 3-manifolds with bounded geometry in $[34 ; 35]$ which was prior to the solution of the general case. (A hyperbolic manifold is said to have bounded geometry if there is a positive lower bound for the lengths of the closed geodesics.) Bromberg's result was then generalised to freely indecomposable Kleinian groups by Brock and Bromberg [9]. The purpose of the present paper is to prove this density conjecture in general, including the case of freely decomposable Kleinian groups. Before explaining what is the difficulty in the freely decomposable case, we shall review the ending lamination conjecture and its resolution, on which our proof of the main result depends. 
The ending lamination conjecture, which is due to Thurston and has been one of the most famous conjectures in the modern Kleinian group theory, says that every topologically tame hyperbolic 3-manifold is determined up to isometries by its homeomorphism type and end invariants consisting of the conformal structures at infinity, parabolic locus and the ending laminations. Here a hyperbolic 3-manifold is said to be topologically tame when it is homeomorphic to the interior of a compact 3-manifold. We shall explain what the end invariants are in more detail. Let $M=\mathbb{H}^{3} / \Gamma$ be a topologically tame hyperbolic 3-manifold, and $M_{0}$ its noncuspidal part. We choose a compact core $C$ such that for each component $T$ of $\partial M_{0}$, the intersection $C \cap T$ is a core of $T$, and each component of $M \backslash C$ is a product open-interval bundle over a component of $\operatorname{Fr} C$, where Fr denotes the frontier. We shall call such a compact core nice. For a nice compact core $C$, we see that $\left(C, C \cap \partial M_{0}\right)$ has a structure of a pared manifold. The parabolic locus of $M$ is $P=C \cap \partial M_{0}$ which consists of annuli and tori. Each component of $\partial C \backslash P$ faces an end of $M_{0}$. The ends of $M_{0}$ have invariants as follows. If an end of $M_{0}$ is geometrically finite, ie has a neighbourhood containing no closed geodesics, then there is a component of the quotient $\Omega_{\Gamma} / \Gamma$ of the region of the discontinuity which can be regarded as the points at infinity of the end, and its marked conformal structure constitutes an invariant for the end. When an end is geometrically infinite, it has an invariant called the ending lamination, which is represented by a measured lamination contained in the Masur domain of the frontier component of $C$ facing the end, determined uniquely up to transverse measures if we fix a marking on the frontier component. (The marking of $\operatorname{Fr} C$ is not uniquely determined by the marking of $M$ when $\operatorname{Fr} C$ has a compressible component.) The ending lamination conjecture says that these pieces of information, the parabolic locus, the marked conformal structure for the geometrically finite ends and the ending laminations for the geometrically infinite ends both up to the action of the auto-diffeomorphisms of the frontier component homotopic to the identity in $C$, together with the homeomorphism type, are sufficient to determine the isometry type of the hyperbolic 3-manifold.

The first essential contribution to solving the ending lamination conjecture was due to Minsky [34; 35] for hyperbolic 3-manifolds with freely indecomposable fundamental groups and bounded geometry. After some work on the unbounded geometry case in special situations as in Minsky [36; 37], the conjecture for the general case when hyperbolic manifolds may have unbounded geometry was finally solved by Minsky [38] and Brock, Canary and Minsky [10] based on the work on the geometry of the curve complex developed by Masur and Minsky [31; 32]. Although Brock, Canary and Minsky [10] only gave a proof for the freely indecomposable case, it can be generalised to the freely decomposable case, as was written in the first section of [10], using the technique of constructing negatively curved metrics on branched covers due to Canary [15]. This 
method in the case of manifolds with bounded geometry corresponding to [34] was explained by the author in [48] and its generalisation to the case of manifolds having the same end invariants as those with bounded geometry, corresponding to [37], was sketched by Ohshika and Miyachi [51].

For the Bers-Sullivan-Thurston density conjecture in the freely decomposable case, it is first necessary to show that every finitely generated Kleinian group is topologically tame so that ending laminations can be defined. This is exactly what Marden's tameness conjecture says, which was recently solved by Agol [2] and Calegari and Gabai [14] independently. Once Marden's conjecture is solved, to show that every (finitely generated and torsion-free) Kleinian group is a limit of quasiconformal deformations of a geometrically finite group, using the resolution of the ending lamination conjecture by Brock, Canary and Minsky, what we need to do is to prove that any possible system of a homomorphism type, a parabolic locus and end invariants can be realised in a Kleinian group which is a limit of minimally parabolic geometrically finite groups. In the case of freely decomposable groups, this consists of two main steps. The first step is to provide a convergence theorem for freely decomposable groups generalising results of Kleineidam and Souto [27], Lecuire [28] and Ohshika [50], the last of which only dealt with Kleinian groups without parabolic elements. The second step is to show that the limit group obtained by the convergence theorem has the desired properties. This in particular necessitates showing that the support of an arational measured lamination contained in the Masur domain, which we know to be unrealisable, is homotopic in $M$ to an ending lamination. This latter step was easy for the freely indecomposable case by work of Thurston and Bonahon, but in our general case, the argument is rather complicated.

We note that Namazi and Souto also have given a proof of this latter step in [43], and have proved the Bers-Sullivan-Thurston density conjecture independently of our work.

We now state in the form of a theorem an affirmative solution of the Bers-SullivanThurston density conjecture for general (topologically tame) Kleinian groups as explained above.

Theorem 1.1 Let $\Gamma$ be a finitely generated, torsion-free Kleinian group. Then there is a geometrically finite Kleinian group $G$ without infinite cyclic maximal parabolic subgroups such that $\Gamma$ is an algebraic limit of quasiconformal deformations of $G$.

Kleinian groups without infinite maximal cyclic parabolic groups are sometimes called minimally parabolic, as in our title. It should be noted that minimally parabolic geometrically finite groups are convex cocompact unless they have rank-2 abelian subgroups. 
This theorem is derived as a corollary from the main theorem of the present paper, which we shall state below, combined with the solution of the ending lamination conjecture and Thurston's uniformisation theorem for compact atoroidal 3-manifolds with boundary (see for instance Morgan [39]). Before stating the theorem formally, we summarise what the theorem says. We consider a minimally parabolic, geometrically finite Kleinian group $G$ and a nice core $C$ of $\left(\mathbb{H}^{3} / G\right)_{0}$. We give on nontorus components of $\partial C$ a union $P$ of disjoint nonparallel essential simple closed curves such that $(C, P)$ is a pared manifold, and on each nontorus component of $\partial C \backslash P$ either a conformal structure or an arational measured lamination contained in the Masur domain, with some conditions which are evidently necessary to make them a parabolic locus and end invariants. Then what we shall claim in the theorem is that there is a topologically tame Kleinian group $\Gamma$ which is an algebraic limit of quasiconformal deformations of $G$ such that $\left(\mathbb{H}^{3} / \Gamma\right)_{0}$ has $P$ as its parabolic locus and the given conformal structures and laminations as its end invariants under the natural marking.

Theorem 1.2 Let $G$ be a torsion-free geometrically finite Kleinian group without infinite cyclic maximal parabolic subgroups. Let $C$ be a nice compact core of $\left(\mathbb{H}^{3} / G\right)_{0}$. Let $T$ denote the union of the torus components of $\partial C$. Let $P$ be a disjoint union of annular neighbourhoods of essential simple closed curves on $\partial C$ such that $(C, P \cup T)$ is a pared manifold. Let $\Sigma_{1}, \ldots, \Sigma_{m}$ be the components of $\partial C \backslash(P \cup T)$. Among $\Sigma_{1}, \ldots, \Sigma_{m}$, suppose that on each $\Sigma_{j}$ with $j=1, \ldots, n$ (possibly $n=0$ ), a marked conformal structure $m_{j}$ making the components of the frontier punctures is given, and that on each $\Sigma_{j}$ with $j=n+1, \ldots, m$ (possibly $m=n$ ), an arational measured lamination $\mu_{j}$ contained in the Masur domain of $\Sigma_{j}$ is given. When $(C, P)$ is a trivial $I$-bundle (as a pared manifold) and $n=0$, we further assume that the supports of $\mu_{1}$ and $\mu_{2}$ are not homotopic in $C$. When $(C, P)$ is a twisted $I$-bundle and $n=0$, we further assume that $\mu_{1}$ is not a lift of a measured lamination in the base space of the $I-$ bundle, which is a nonorientable surface.

Then there is a sequence of quasiconformal deformations $G_{i}$ of $G$ with isomorphisms $\phi_{i}: G \rightarrow G_{i}$ converging algebraically to an isomorphism $\psi: G \rightarrow \Gamma$ to a topologically tame Kleinian group $\Gamma$ such that $\left(\mathbb{H}^{3} / \Gamma\right)_{0}$ has a nice compact core $C^{\prime}$ with a homeomorphism $\Phi: C \rightarrow C^{\prime}$ inducing $\phi$ between the fundamental groups, such that

(i) $\Phi(P \cup T)$ coincides with the parabolic locus of $C^{\prime}$ regarded as a pared manifold,

(ii) the end of $\left(\mathbb{H}^{3} / \Gamma\right)_{0}$ facing $\Phi\left(\Sigma_{j}\right)$ with $j=1, \ldots, n$ is geometrically finite and has marked conformal structure at infinity equal to the one represented by $\Phi_{*}\left(m_{j}\right)$,

(iii) the end of $\left(\mathbb{H}^{3} / \Gamma\right)_{0}$ facing $\Phi\left(\Sigma_{j}\right)$ with $j=n+1, \ldots, m$ is geometrically infinite with ending lamination represented by $\Phi\left(\mu_{j}\right)$. 
We should note that this theorem can be also regarded as a generalisation of the main theorem of Ohshika [47], where we generalised Maskit's theorem in [30] on function groups to one on general geometrically finite groups.

The proof of this theorem will proceed as follows. We shall first construct quasiconformal deformations $\left(G_{i}, \phi_{i}\right)$ of $G$ so that the conformal structure at infinity of $\mathbb{H}^{3} / G_{i}$ restricted to $\Sigma_{j}$ converges to $m_{j}$ for $j=1, \ldots, n$ and diverges towards $\left[\mu_{j}\right]$ in the Thurston compactification of the Teichmüller space for $j=n+1, \ldots m$, and the length of $\mu_{j}$ with respect to the hyperbolic metric compatible with the conformal structure $m_{j}$ is bounded. The first of the two main steps is to show that such a sequence of quasiconformal structures converges algebraically after passing to a subsequence. The proof of this fact relies on work of Otal [52], Kleineidam and Souto [27] and a more recent result of Lecuire [28] on the extension of the Masur domain.

The second main step is to show that the limit group has properties that we wanted. It follows from results of Ohshika [49] and Brock and Souto [11] (or the general solution of Marden's conjecture) that the limit $\Gamma$ is topologically tame, and using some geometric argument, we can show that the $\mu_{j}$ cannot be realised there. The most difficult part of the second step is to show that (the images by a homeomorphism from $\mathbb{H}^{3} / G$ to $\mathbb{H}^{3} / \Gamma$ of) the $\mu_{j}$ actually represent ending laminations (Proposition 6.5). Section 6 will be entirely devoted to the proof of this fact.

Acknowledgements The author is grateful to the referee for his/her careful reading and valuable comments.

\section{Preliminaries}

Throughout this paper, Kleinian groups are assumed to be finitely generated and torsion free except for the case when we consider geometric limits, which may be infinitely generated. Similarly, we always consider hyperbolic 3-manifolds with finitely generated fundamental groups except for geometric limits. For a Kleinian group $G$, we consider the corresponding hyperbolic 3-manifold $M_{G}=\mathbb{H}^{3} / G$. Throughout this paper, we use the symbol $M$ with the name of a Kleinian group added as a subscript to denote the corresponding hyperbolic 3-manifold. For a constant $\epsilon>0$ less than the three-dimensional Margulis constant, we define the noncuspidal part, denoted by $\left(M_{G}\right)_{0}$, to be the complement of the $\epsilon$-cusp neighbourhoods of $M_{G}$, that is, neighbourhoods of cusps consisting of points where the injectivity radii are less than $\epsilon / 2$.

Since $G$ is assumed to be finitely generated, by Scott's core theorem [55], there is a compact 3-manifold $C_{G}$, which we call a compact core, embedded in $M_{G}$ such that 
the inclusion from $C_{G}$ to $M_{G}$ is a homotopy equivalence. When $G$ has parabolic elements, it is more convenient to consider a relative compact core of the noncuspidal part $\left(M_{G}\right)_{0}$, whose existence was proved by McCullough [33]. A relative compact core intersects the boundary of the noncuspidal part at tori corresponding to $\mathbb{Z} \times \mathbb{Z}$-cusps and annuli which are cores of open annuli corresponding to $\mathbb{Z}$-cusps, one annulus for each $\mathbb{Z}$-cusp. The intersection with the boundary of the noncuspidal part induces a structure of pared manifold on a relative compact core, which we shall explain below.

A Kleinian group $G$ and the corresponding hyperbolic 3-manifold $M_{G}$ are said to be topologically tame when $M_{G}$ is homeomorphic to the interior of a compact 3-manifold. In this case, we can choose a relative compact core $C_{G}$ so that each component $E$ of $M_{G} \backslash C_{G}$ is homeomorphic to $F \times \mathbb{R}$, where $F$ is the component of $\operatorname{Fr} C_{G}$ contained in the closure of $E$. As was mentioned in Introduction, we call such a compact core nice.

Following Thurston, we call a pair $(C, P)$ of a compact irreducible 3-manifold and a subsurface of its boundary a pared manifold when

(i) $P$ consists of disjoint incompressible tori and annuli,

(ii) every incompressible (ie $\pi_{1}$-injective) map from a torus to $C$ is homotopic into $P$,

(iii) every incompressible map $\left(S^{1} \times I, S^{1} \times \partial I\right) \rightarrow(C, P)$ is relatively homotopic to a map into $P$.

The subsurface $P$ above is called the paring locus. When we consider a pared manifold which is a relative compact core of the noncuspidal part of a hyperbolic 3-manifold, we call its paring locus the parabolic locus.

A compression body $W$ is a connected 3-manifold obtained from finitely many product $I$-bundles $S_{1} \times I, \ldots, S_{m} \times I$ over closed surfaces which are not spheres by attaching 1 -handles to $\cup S_{k} \times\{1\}$. We assume that there is at least one 1-handle; hence we do not regard a product $I$-bundle as a compression body. Exceptionally handlebodies are also regarded as compression bodies. The union of the $S_{k} \times\{0\}$, called the interior boundary, is denoted by $\partial_{i} W$, and the remaining boundary component coming from $S_{k} \times\{1\}$, which is called the exterior boundary, is denoted by $\partial_{e} W$. We use the same symbols $\partial_{e}$ and $\partial_{i}$ to denote the unions of the exterior boundaries and the interior boundaries respectively for a disjoint union of compression bodies. For a compact irreducible 3-manifold $C$, there exists a submanifold $V$ which is a disjoint union of compression bodies such that $V \cap \partial C=\partial_{e} V$ is the union of compressible boundary components of $C$, and each component of $\partial_{i} V$ (unless it is empty) is either an incompressible surface in Int $C$ which is not parallel into $\partial C$ or an incompressible boundary component of $C$. Such a manifold is unique up to isotopy and is called 
the characteristic compression body of $C$. If $V$ is a characteristic compression body of $C$, then every incompressible (ie $\pi_{1}$-injective) map from a closed surface to $C$ is homotoped into $(C \backslash V) \cup \partial_{i} V$. The closure of $C \backslash V$ is a (possibly empty) boundaryirreducible manifold and none of its components are product $I$-bundles unless $C$ itself is a product $I$-bundle. These facts are proved by Bonahon [6], to which we refer the reader for further details.

Let $S$ be a hyperbolic surface, possibly with geodesic boundary. A geodesic lamination on $S$ is a closed set disjoint from $\partial S$ consisting of disjoint simple geodesics. A measured lamination is a geodesic lamination with a holonomy-invariant transverse measure. We always assume that the support of the measure is the entire lamination. For a measured lamination $\mu$, its support is denoted by $|\mu|$. The space of measured laminations with the weak topology on transverse arcs is denoted by $\mathcal{M L}(S)$. The projective lamination space is $(\mathcal{M L}(S) \backslash\{\varnothing\}) / \mathbb{R}_{+}$, where the action of $\mathbb{R}_{+}$is that of scalar multiplication of the transverse measures, and is denoted by $\mathbb{P} \mathcal{M L}(S)$. Thurston defined a compactification of the Teichmüller space $\mathcal{T}(S)$ whose boundary is identified with $\mathbb{P} \mathcal{M L}(S)$. When $S$ has boundary, we define its Teichmüller space to be the space of marked hyperbolic structures with respect to which the lengths of the boundary components are constant. We sometimes say that $m_{i} \in \mathcal{T}(S)$ diverges towards $\lambda \in \mathbb{P} \mathcal{M L}(S)$ when $\left\{m_{i}\right\}$ converges to $\lambda$ in the Thurston compactification.

A geodesic lamination is said to be arational when every component of its complement is either simply connected, or an annulus around a cusp or a boundary component. An arational measured lamination is always minimal, ie, it does not have a proper sublamination. We say that a measured lamination is maximal when it is not a proper sublamination of another measured lamination. Arational measured laminations are always maximal.

Consider a compact 3-manifold $C$ and an essential subsurface $S$ contained in a component of $\partial C$. We assume that no boundary components of $S$ are meridians (ie boundaries of compressing discs). In the measured lamination space $\mathcal{M L}(S)$, we define the following subsets. First, we set $\mathcal{W C}(S)$ to be the subset of $\mathcal{M L}(S)$ consisting of disjoint weighted union of meridians lying on $S$. Except for the case when $S$ has only one isotopy class of compressing discs, we define the Masur domain of $S$ by

$$
\mathcal{M}(S)=\{\lambda \in \mathcal{M L}(S) \mid i(\lambda, v)>0 \text { for any } v \in \overline{\mathcal{W C}}(S)\},
$$

where $\overline{\mathcal{W C}}(S)$ denotes the closure of $\mathcal{W C}(S)$ in $\mathcal{M L}(S)$. When $S$ has only one isotopy class of compressing discs, we define the Masur domain by

$\mathcal{M}(S)=\{\lambda \in \mathcal{M L}(S) \mid i(\lambda, v)>0$, for any $v$ that is disjoint from a meridian $\}$. 
We note that $\mathcal{M}(S)$ coincides with the entire measured lamination space if $S$ is incompressible.

We need to define another domain in $\mathcal{M L}(\partial V)=\mathcal{M L}\left(\partial_{e} V\right) \cup \mathcal{M L}\left(\partial_{i} V\right)$ larger than the Masur domain when $V$ is a compression body, following Lecuire [28]. For a compression body $V$, we set

$$
\begin{aligned}
\mathcal{D}(V)=\{\lambda \in \mathcal{M L}(\partial V) \mid & \text { there exists } \eta>0 \text { such that } i(\lambda, \partial D)>\eta \\
& \text { for any compression disc and any essential annulus } D\} .
\end{aligned}
$$

The subspaces $\mathcal{W C}(S), \mathcal{M}(S)$ and $\mathcal{D}(V)$ are all invariant under scalar multiplication. We put $\mathbb{P}$ to denote their images in the projective lamination space $\mathbb{P} \mathcal{M L}(S)$ or $\mathbb{P} \mathcal{M L}(\partial V)$.

The subgroup of the mapping class group of $S$ consisting of classes of diffeomorphisms homotopic to the identity in $V$ is denoted by $\operatorname{Mod}_{0}(V)$. This group $\operatorname{Mod}_{0}(V)$ acts on $\mathbb{P} \mathcal{M}(S)$ properly discontinuously, and the limit set of $\operatorname{Mod}_{0}(V)$ in $\mathbb{P} \mathcal{M L}(S)$ is equal to $\overline{\mathbb{P} \mathcal{W C}}(S)$. (Refer to Otal [52].)

We let $\widetilde{V}$ be the universal cover of $V$ and $\widetilde{S}$ the preimage of $S$ lying on $\partial \widetilde{V}$. We fix a hyperbolic metric on $S$, which induces one on $\widetilde{S}$. Let $l$ be a leaf of a geodesic lamination $\mu$ on $S$. Consider a lift $\tilde{l}: \mathbb{R} \rightarrow \widetilde{S}$ of $l$. We say that $l$ is homoclinic if there are sequences of points $\left\{s_{i}\right\},\left\{t_{i}\right\}$ on $\mathbb{R}$ such that $\left|s_{i}-t_{i}\right| \rightarrow \infty$ whereas $d_{\tilde{S}}\left(\widetilde{l}\left(s_{i}\right), \tilde{l}\left(t_{i}\right)\right)$ is bounded above.

We need to use the following lemma of Otal [52]. (This is contained in the proof of Proposition 2.10 of [52]. Kleineidam and Souto stated this as Lemma 4 in [27].)

Lemma 2.1 The support of a measured lamination contained in $\mathcal{M}(S)$ cannot be extended to a geodesic lamination with a homoclinic leaf.

For a hyperbolic 3-manifold $M$, there exists a unique minimal convex submanifold that is a deformation retract. Such a submanifold is called the convex core of $M$. When the convex core of $M_{G}$ is compact, $G$ is said to be convex cocompact, and when the convex core has finite volume, $G$ is said to be geometrically finite.

More generally, for a hyperbolic 3-manifold $M$, an end of $M_{0}$ is said to be geometrically finite when it has a neighbourhood intersecting no closed geodesics.

By the resolution of Marden's conjecture, we can take a nice compact core $C$ of $M_{0}$ so that each end $e$ has a neighbourhood homeomorphic to $\Sigma \times \mathbb{R}$ for a component $\Sigma$ of $\operatorname{Fr} C$ facing $e$. Suppose that $e$ is geometrically infinite. By Proposition 10.1 in Canary [16], there is a sequence of closed geodesics $c_{k}^{*}$ tending to $e$ such that $c_{k}^{*}$ is 
homotopic in $\Sigma \times[0, \infty)$ to a simple closed curve $c_{k}$ on $\Sigma$ such that $\left\{r_{k} c_{k}\right\}$ converges to a measured lamination $\mu$ in $\mathcal{M}(\Sigma)$ for some $r_{k} \in(0, \infty)$. In this situation, we say that $\mu$ represents the ending lamination of $e$. Actually the transverse measure is irrelevant for the ending lamination. The geodesic lamination which is the support of a measured lamination representing the ending lamination is called the ending lamination of $e$.

A pleated surface is a map $f: S \rightarrow M$ from a hyperbolic surface to a hyperbolic 3-manifold taking a puncture or a boundary component to a cusp such that for any point $x \in S$, there is a geodesic on $S$ passing through $x$ which is mapped to a geodesic in $M$, and the length metric on $S$ induced by $f$ from $M$ coincides with that induced from the hyperbolic metric on $S$. When we consider a component $S$ of $\partial C \backslash P$ for some pared manifold $(C, P)$, we always assume that pleated surfaces map frontier components of $S$ to cusps of $M$. In some situation, we need to consider a pleated surface taking each boundary component to a closed geodesic. We call such a pleated surface a pleated surface with boundary. A geodesic lamination $\lambda$ on $S$ is said to be realised by a pleated surface $f$ when $f \mid \lambda$ is totally geodesic.

The following dichotomy for measured laminations in the Masur domain was proved by Otal [52]. See Section 2, above all, Théorème 2.2 of [52].

Lemma 2.2 Let $(C, P)$ be a pared manifold and $S$ a component of $\partial C \backslash P$. Consider a map $g: S \rightarrow M$ to a hyperbolic 3-manifold $M$ sending the frontier of $S$ to cusps of $M$. Let $\lambda$ be a measured lamination contained in the Masur domain of $S$. Then one and only one of the following holds:

(i) Either $\lambda$ is realised by a pleated surface homotopic to $g$ keeping the frontier mapped to cusps, or

(ii) for any sequence of weighted simple closed curves $\left\{r_{k} c_{k}\right\}$ on $S$ converging to $\lambda$, the closed geodesics $c_{k}^{*}$ freely homotopic to $g\left(c_{k}\right)$ tend to an end of $M_{0}$ after passing to a subsequence.

For a geodesic or measured lamination $\lambda$ on $\partial C$, a subsurface of $\partial C$ containing $\lambda$ without boundary components null-homotopic on $\partial C$ which is minimal up to isotopies is called the minimal supporting surface of $\lambda$ and is denoted by $T(\lambda)$. In a special case when $\lambda$ is a (weighted) simple closed curve, we define its minimal supporting surface to be its annular neighbourhood. The minimal supporting surface is unique up to isotopy.

We shall next define for two geodesic laminations on the boundary of a 3-manifold to be isotopic. In contrast to the case of simple closed curves, we need to take more care since without specifying surfaces on which lamination lie, the meaning of isotopy 
for laminations is not so clear. Two disjoint minimal geodesic laminations $\lambda_{1}, \lambda_{2}$ on $\partial C$ are said to be isotopic when there is a sequence of properly embedded essential annuli $A_{j}$ such that $\partial A_{j}$ converges in the Hausdorff topology to a geodesic lamination on $T\left(\lambda_{1}\right) \cup T\left(\lambda_{2}\right)$ containing $\lambda_{1} \cup \lambda_{2}$ as $j \rightarrow \infty$. In the case when no boundary component of $T\left(\lambda_{1}\right)$ and $T\left(\lambda_{2}\right)$ are meridians, this definition of isotopic laminations implies that they lie on homotopic minimal supporting surfaces as we shall see below.

Lemma 2.3 Let $\lambda_{1}$ and $\lambda_{2}$ be two disjoint minimal geodesic laminations on $\partial C$ which are isotopic. Suppose that no boundary component of $T\left(\lambda_{1}\right)$ and $T\left(\lambda_{2}\right)$ is a meridian. Suppose moreover that $\lambda_{i}$ is contained the Masur domain of $T\left(\lambda_{i}\right)$ for $i=1,2$. Then the following hold.

(i) The minimal supporting surfaces $T\left(\lambda_{1}\right), T\left(\lambda_{2}\right)$ of $\lambda_{1}$ and $\lambda_{2}$ are both incompressible in $C$.

(ii) $T\left(\lambda_{1}\right)$ is homotopic to $T\left(\lambda_{2}\right)$ in $C$.

Proof (i) Suppose that $T\left(\lambda_{1}\right)$ is compressible seeking a contradiction. Let $\left\{A_{j}\right\}$ be a sequence of essential annuli whose intersection with $\partial C$ converge to $\lambda_{1} \cup \lambda_{2}$ in the Hausdorff topology. As was shown by Morgan and Shalen [42], there is an incompressible branched surface $B$ properly embedded in $C$ which carries all the annuli $A_{i}$. Moreover, it was shown there that such a branched surface can be chosen so that $B \cap \partial C \subset T\left(\lambda_{1}\right) \cup T\left(\lambda_{2}\right)$, and $B=\tau \times I$ for some train track $\tau$ such that $\tau \times\{0\}$ carries $\lambda_{1}$ and $\tau \times\{1\}$ does $\lambda_{2}$. By choosing a train track approximating $\lambda_{1}$ closely enough and removing branches redundant for carrying $\lambda_{1}$, we can make $B$ carry no compressing discs for $T\left(\lambda_{1}\right)$ since $\lambda_{1}$ is contained in the Masur domain. Since $B$ is both incompressible and boundary-incompressible, by the standard cut-and-paste argument (which uses discs and semidiscs) starting from some compressing disc for $T\left(\lambda_{1}\right)$, we see that we can reduce the intersection with $B$, and finally there must be a compressing disc $D$ contained in $T\left(\lambda_{1}\right)$ disjoint from $B$. Since $\lambda_{1}$ is arational in $T\left(\lambda_{1}\right)$ and carried by $B$, this is possible only when such a disc is isotopic to a boundary component of $T\left(\lambda_{1}\right)$. This contradicts our assumption, and we have thus shown that $T\left(\lambda_{1}\right)$ is incompressible. The same argument works for $T\left(\lambda_{2}\right)$.

(ii) By (i), we know that both $T\left(\lambda_{1}\right)$ and $T\left(\lambda_{2}\right)$ are incompressible. We can assume that they are disjoint by moving their boundaries slightly by an isotopy if they intersect each other at their boundaries since $\lambda_{1}$ and $\lambda_{2}$ are assumed to be disjoint. We can then apply the Jaco-Shalen-Johannson theory $[24 ; 25]$ to $\left(C, T\left(\lambda_{1}\right) \cup T\left(\lambda_{2}\right)\right)$ to obtain a characteristic $I$-pair $(\Sigma, \Phi)$ properly embedded in $\left(C, T\left(\lambda_{1}\right) \cup T\left(\lambda_{2}\right)\right)$ such that any essential annulus properly embedded in $\left(C, T\left(\lambda_{1}\right) \cup T\left(\lambda_{2}\right)\right)$ is properly homotopic into $(\Sigma, \Phi)$. Since $\lambda_{1}$ is arational in $T\left(\lambda_{1}\right)$ and there is an essential annulus whose 
intersection with $T\left(\lambda_{1}\right)$ approximates $\lambda_{1}$ arbitrarily closely, we see that $\Phi \cap T\left(\lambda_{1}\right)$ must be isotopic to $T\left(\lambda_{1}\right)$. The same holds for $T\left(\lambda_{2}\right)$. Since $\Sigma$ is an $I$-bundle, this means that $T\left(\lambda_{1}\right)$ and $T\left(\lambda_{2}\right)$ cobound a product $I$-bundle in $C$.

An $\mathbb{R}$-tree is a geodesic metric space in which two points are connected by a unique simple arc. An isometric action of a group $G$ on an $\mathbb{R}$-tree $T$ is said to have small edge-stabilisers when for any nontrivial segment $c$ of $T$, the stabiliser of $c$ is either a finite group or a finite extension of $\mathbb{Z}$. Let $S$ be a hyperbolic surface of finite area, and suppose that there is an action of $\pi_{1}(S)$ on an $\mathbb{R}$-tree $T$ by isometries. A geodesic lamination $\lambda$ on $S$ is said to be realised in $T$ when there is an equivariant map from the universal cover $\mathbb{H}^{2}$ of $S$ to $T$ which maps each component of the preimages of the leaves of $\lambda$ injectively. It was proved by Otal [53] that if a measured lamination $\lambda$ is realised, then there is a train track $\tau$ carrying $\lambda$ which is realised by the same equivariant map: that is, there is an equivariant map from the universal cover $\tilde{\tau}$ of $\tau$ such that every branch of $\tilde{\tau}$ is mapped to a nondegenerate segment and any train route is mapped locally injectively at every switch of $\tilde{\tau}$.

For a Kleinian group $G$, we define its deformation space to be the space of faithful discrete representations of $G$ into $\mathrm{PSL}_{2} \mathbb{C}$ modulo conjugacy. We endow this space with the induced topology as a quotient space of the representation space with the topology of pointwise convergence, and denote it by $A H(G)$. We denote an element of $A H(G)$, which is a conjugacy class of representations, by a representation $\phi: G \rightarrow \mathrm{PSL}_{2} \mathbb{C}$ representing the conjugacy class or by a pair $(\Gamma, \phi)$, where $\Gamma$ is the Kleinian group $\phi(G) \subset \mathrm{PSL}_{2} \mathbb{C}$. The quasiconformal deformations of $G$ modulo conjugacy form a subspace of $A H(G)$, which is denoted by $Q H(G)$.

A sequence of Kleinian groups $\left\{G_{i}\right\}$ is said to converge geometrically to a Kleinian group $H$ if every element of $H$ is the limit of a sequence $\left\{g_{i}\right\}$ for $g_{i} \in G_{i}$, and the limit of any convergent sequence $\left\{g_{i_{j}} \in G_{i_{j}}\right\}$ for a subsequence $\left\{G_{i_{j}}\right\} \subset\left\{G_{i}\right\}$ is contained in $H$. If $\phi_{i}: G \rightarrow \mathrm{PSL}_{2} \mathbb{C}$ converges to $\phi$ as representations, then its images $\phi_{i}(G)$ converge geometrically (up to extracting a subsequence) to a Kleinian group containing $\phi(G)$. When the geometric limit and the algebraic limit coincide, we say that the convergence is strong.

Suppose that a sequence of Kleinian groups $\left\{G_{i}\right\}$ converges to $H$ geometrically. Let $p_{i}: \mathbb{H}^{3} \rightarrow M_{G_{i}}$ and $q: \mathbb{H}^{3} \rightarrow M_{H}$ be universal coverings. Fix a point $x$ in $\mathbb{H}^{3}$. Then, $\left(M_{G_{i}}, p_{i}(x)\right)$ converges to $\left(M_{H}, q(x)\right)$ in the pointed Gromov-Hausdorff topology: that is, there exists a $K_{i}$-bi-Lipschitz map to its image $\rho_{i}: B_{r_{i}}\left(M_{G_{i}}, p_{i}(x)\right) \rightarrow$ $B_{K_{i} r_{i}}\left(M_{H}, q(x)\right)$ with $K_{i} \rightarrow 1$ and $r_{i} \rightarrow \infty$. In this situation, we say a map $f_{i}: N_{i} \rightarrow M_{G_{i}}$ from some Riemannian manifold $N_{i}$ converges geometrically to a 
map $g: N^{\prime} \rightarrow M_{H}$ if $\left(N_{i}, z_{i}\right)$ converges to $\left(N^{\prime}, z^{\prime}\right)$ geometrically with some basepoint $z_{i}$ which has bounded $d_{M_{G_{i}}}\left(p_{i}(x), z_{i}\right)$ and with approximate isometries $\bar{\rho}_{i}$, and $\rho_{i} \circ f_{i} \circ \bar{\rho}_{i}^{-1}$ converges to $g$ uniformly on every compact subset of $N^{\prime}$.

\section{Construction of sequences and the statement of the conver- gence theorem}

Consider a minimally parabolic, geometrically finite Kleinian group $G$ as was given in Theorem 1.2. Let $S_{1}, \ldots, S_{p}$ be the boundary components of $C$ that are not tori. We shall define a sequence of marked conformal structures $\left\{n_{i}^{k}\right\}$ on each component $S_{k}$. First consider core curves of $P \cap S_{k}$, one on each component of $P \cap S_{k}$, and denote them by $c_{1}^{k}, \ldots, c_{q}^{k}$ and their union by $C^{k}$. On each component of $S_{k} \backslash C^{k}$, either a marked conformal structure or a measured lamination is given in Theorem 1.2. We denote the components of $S_{k} \backslash C^{k}$ on which marked conformal structures are given by $\Sigma_{1}^{k}, \ldots, \Sigma_{s}^{k}$, and the given marked conformal structures by $m_{1}^{k}, \ldots, m_{s}^{k}$. Let $\mu_{s+1}^{k}, \ldots, \mu_{r}^{k}$ be the measured laminations given on the rest of the components $\Sigma_{s+1}^{k}, \ldots, \Sigma_{r}^{k}$, and $M^{k}$ their union. Sometimes it is more convenient to consider a compact surface which is obtained by deleting a collar neighbourhood of the frontier from $\Sigma_{j}^{k}$ than $\Sigma_{j}^{k}$ itself. Slightly abusing notation, we use the same symbol $\Sigma_{j}^{k}$ to denote such a compact surface.

Definition 3.1 We define marked hyperbolic structures $n_{i}^{k}$ on $S_{k}$ in such a way that

(i) length $n_{i}^{k}\left(c_{j}^{k}\right)=1 / i$ for every $j=1, \ldots, q$,

(ii) length $n_{i}^{k}\left(\mu_{j}^{k}\right)$ is bounded above independently of $i$ for every $j=s+1, \ldots, r$,

(iii) $\left\{n_{i}^{k} \mid \Sigma_{j}^{k}\right\}$ diverges in the Teichmüller space of $\Sigma_{j}^{k}$ towards the projective lamination $\left[\mu_{j}^{k}\right]$ on the Thurston boundary for $j=s+1, \ldots, r$,

(iv) $\left\{n_{i}^{k} \mid \Sigma_{j}^{k}\right\}$ converges to $m_{j}^{k}$ as $i \rightarrow \infty$ for every $j=1, \ldots, s$.

Lemma 3.1 Hyperbolic structures $n_{i}^{k}$ satisfying the four conditions above exist.

Proof We shall construct hyperbolic structures on each $\Sigma_{j}^{k}$, and get structures as we want by pasting them together. First we consider $\Sigma_{j}^{k}$ for $j=1, \ldots, s$. The conformal structure $m_{j}^{k}$ is realised by a hyperbolic structure making the frontier of $\Sigma_{j}^{k}$ cusps. Such a structure is approximated by hyperbolic structures with respect to which every component of $\partial \Sigma_{j}^{k}$ is a closed geodesic of length $\delta$ with $\delta \rightarrow 0$. Therefore we can choose a hyperbolic structure $n_{i}^{k}(j)$ converging to $m_{j}^{k}$ on $\Sigma_{j}^{k}$ so that each component of $\partial \Sigma_{j}^{k}$ is a closed geodesic of length $1 / i$. 
Next we consider $\Sigma_{j}^{k}$ for $j=s+1, \ldots, r$. For each $j$, take a sequence of hyperbolic structures $\left\{n_{i}^{\prime j}\right\}$ converging to a complete hyperbolic structure $n^{\prime j}$ making $\partial \Sigma_{j}^{k}$ cusps such that the length of each component of $\partial \Sigma_{j}^{k}$ with respect to $n_{i}^{\prime j}$ is $1 / i$. We should note that since $\left\{n_{i}^{\prime}\right\}$ converges to $n_{0}^{\prime j}$, the lengths of $\mu_{j}^{k}$ with respect to the $n_{i}^{\prime j}$ are bounded above. We let $n_{i}^{k}(j)$ be the hyperbolic structure obtained from $n_{i}^{\prime j}$ by the earthquake along $i \mu_{j}^{k}$, which is a measured lamination with the transverse measure of $\mu_{j}^{k}$ multiplied by $i$. Then we have

$$
\text { length }_{n_{i}^{k}(j)}\left(\mu_{j}^{k}\right)=\text { length }_{n_{i}^{\prime j}}\left(\mu_{j}^{k}\right),
$$

which is bounded above independently of $i$. Also, since $\left\{n_{i}^{\prime j}\right\}$ converges and the earthquake is performed along $i \mu_{j}^{k}$, we see that $\left\{n_{i}^{k}(j)\right\}$ diverges towards the projective lamination represented by $\mu_{j}^{k}$ on the Thurston boundary of $\mathcal{T}\left(\Sigma_{j}^{k}\right)$.

Pasting these hyperbolic structures $n_{i}^{k}(j)$ along the $c_{j}^{k}$, we get a hyperbolic structure as we wanted.

For a sequence satisfying the four conditions, we have the following.

Lemma 3.2 Let $\left\{n_{i}^{k}\right\}$ be a sequence of hyperbolic structures on $S_{k}$ satisfying the four conditions above. Then for each $j=s+1, \ldots, r$, there exists a sequence of weighted simple closed curves $\left\{r_{i} c_{i}\right\}$ on $\Sigma_{j}^{k}$ with the following two properties.

(i) length $n_{i}^{k}\left(r_{i} c_{i}\right)$ goes to 0 as $i \rightarrow \infty$.

(ii) $\left\{r_{i} c_{i}\right\}$ converges to a measured lamination having the same support as $\mu_{j}^{k}$.

Proof Let $c_{i}$ be the shortest nonperipheral closed geodesic on $\Sigma_{j}^{k}$ with respect to the metric $n_{i}^{k}$. By Bers' theorem (see [4]), there is a constant $K$ bounding the length $n_{i}^{k}\left(c_{i}\right)$ from above. Taking a subsequence, we can assume that there is a sequence of positive numbers $r_{i}$ such that $\left\{r_{i} c_{i}\right\}$ converges to a nonempty measured lamination $v_{j}^{k}$ on $\Sigma_{j}^{k}$. Note that $r_{i}$ goes to 0 if $c_{i}$ is not eventually constant. Even in the latter exceptional case, $r_{i}$ is bounded above.

We shall show that $\nu_{j}^{k}$ and $\mu_{j}^{k}$ have the same support. Suppose that they do not, seeking a contradiction. Since $\mu_{j}^{k}$ was assumed to be arational, then we have $i\left(\mu_{j}^{k}, v_{j}^{k}\right)>0$. Since $n_{i}^{k} \mid \Sigma_{j}^{k}$ diverges towards $\left[\mu_{j}^{k}\right]$, this implies, by Lemma 8.II.1 in Fathi, Laudenbach and Poénaru [21], that length $n_{i}^{k}\left(r_{i} c_{i}\right) \rightarrow \infty$. Since $r_{i}$ is bounded above, it follows that length $_{n_{i}^{k}}\left(c_{i}\right)$ also goes to $\infty$. This contradicts the fact that the lengths are bounded above by $K$.

Since $\mu_{j}^{k}$ is not a closed geodesic, neither is $v_{j}^{k}$, and we see that $r_{i} \rightarrow 0$. Hence length $_{n_{i}^{k}}\left(r_{i} c_{i}\right) \leq r_{i} K \rightarrow 0$. Thus we have completed the proof. 
Let $Q H(G)$ be the subset of $A H(G)$ consisting of points in $A H(G)$ represented by quasiconformal deformations of $G$, endowed with the relative topology induced from $A H(G)$. Let $q: \mathcal{T}\left(\Omega_{G} / G\right) \rightarrow Q H(G)$ be the Ahlfors-Bers map, which is a (possibly ramified) covering map. This map is obtained as follows. For any point $m \in \mathcal{T}\left(\Omega_{G} / G\right)$, there is a Beltrami differential $\mu$ on $\Omega_{G}$ giving rise to a quasiconformal homeomorphism from the original conformal structure on $\Omega_{G} / G$ to $\mu$, which is automorphic under the action of $G$. We extend $\mu$ to the entire Riemann sphere by setting the value outside $\Omega_{G}$ to be 0 , which is obviously also automorphic. This defines a quasiconformal deformation of $G$, which we set $q(m)$ to be.

Recall that $\Omega_{G} / G$ is topologically identified with the union of nontorus components of $\partial C$.

Definition 3.2 For $n_{i}^{1}, \ldots, n_{i}^{p}$ defined in Definition 3.1, we let $\left\{v_{i}=\left(n_{i}^{1}, \ldots, n_{i}^{p}\right)\right\}$ be a sequence regarded as lying in $\mathcal{T}\left(\Omega_{G} / G\right)$.

One of the key ingredients to show our main theorem is the following.

Theorem 3.3 The sequence $\left\{q\left(v_{i}\right)\right\}$ converges in the deformation space $A H(G)$, after passing to a subsequence.

We shall prove this theorem in the following two sections. The proof is based on the work of Kleineidam and Souto [27], Lecuire [28] and Ohshika [50]. Although there is another way to use more general result by Kim, Lecuire and Ohshika [26] which is still unpublished, we have chosen to use only [27; 28; 50] since the argument in [26] is much more complicated than these three. We note that even if we invoke the main theorem of [26], we cannot obtain Theorem 3.3 immediately and the general line of our argument does not change, although some part can be shorten.

Let $\phi_{i}: G \rightarrow \mathrm{PSL}_{2}(\mathbb{C})$ be a geometrically finite representation corresponding to $q\left(v_{i}\right)$, and denote its image by $G_{i}$, which is a geometrically finite group. Since $\phi_{i}$ is induced by a quasiconformal deformation, there is a homeomorphism $\Phi_{i}: M_{G} \rightarrow M_{\phi_{i}(G)}$ inducing the isomorphism $\phi_{i}$ between the fundamental groups.

\section{Limit laminations of the boundary-irreducible part}

In [50], we first analysed the hyperbolic structures on the characteristic compression body. In contrast, in the present argument, we begin by analysing the behaviour of the hyperbolic structures on the complement of the characteristic compression body. 
Consider the characteristic compression body $V$ of $C$. We call the closure of the complement of $V$ the boundary-irreducible part of $C$. Let $W$ be a component of the boundary-irreducible part. Then $\pi_{1}(W)$ injects to $\pi_{1}(C) \cong G$ by the homomorphism induced from the inclusion. We denote its image by $H^{W}$.

We recall the following lemma essentially due to Thurston. Its detailed proof can be found in Theorem 3.1 of Ohshika [50] except for the last sentence concerning the Hausdorff convergence. It should be also noted that although it was assumed that the Kleinian group does not have parabolic elements in [50], the existence of rank-2 parabolic subgroups does not affect its proof at all.

We note that the codimension-one measured lamination which we get in this lemma describes only the behaviour of the lengths of the simple closed curves on $\partial W$. It may not capture the behaviour of the lengths of the closed curves in $W$ in general, hence may not be a lamination dual to the action of $\pi_{1}(W)$ on an $\mathbb{R}$-tree which is a rescaled Gromov limit of $\left(\Gamma_{i}, \psi_{i}\right)$. Remark 3.2 in [50] gave such an example.

Lemma 4.1 Let $H$ be a minimally parabolic, freely indecomposable Kleinian group. Let $W$ be a compact core of $M_{H}$. Suppose we have a sequence $\left\{\left(\Gamma_{i}, \psi_{i}\right) \in A H(H)\right\}$ which does not have a convergent subsequence. We denote a homotopy equivalence from $M_{H}$ to $M_{\Gamma_{i}}$ inducing $\psi_{i}$ between the fundamental groups by $\Psi_{i}$. Then, after passing to a subsequence of $\left\{\left(\Gamma_{i}, \psi_{i}\right)\right\}$, there is a sequence of disjoint, nonparallel unions of essential annuli $\left\{A_{i}^{1} \sqcup \ldots \sqcup A_{i}^{\kappa}\right\}$ properly embedded in $W$, whose number $\kappa$ is independent of $i$, with positive weights $w_{i}^{1}, \ldots, w_{i}^{\kappa}$ such that $w_{i}^{1} A_{i}^{1} \sqcup \cdots \sqcup w_{i}^{\kappa} A_{i}^{\kappa}$ converges in the measure topology to some codimension-1 measured lamination in $W$ and the following hold. For any two convergent sequences of measured laminations $\alpha_{i}, \beta_{i}$ lying on $\partial W$, we have

$$
\lim _{i \rightarrow \infty} \frac{\operatorname{length}\left(\Psi_{i}\left(\alpha_{i}\right)\right)}{\operatorname{length}\left(\Psi_{i}\left(\beta_{i}\right)\right)}=\lim _{i \rightarrow \infty} \frac{i\left(\alpha_{i}, w_{i}^{1} A_{i}^{1}\right)+\cdots+i\left(\alpha_{i}, w_{i}^{\kappa} A_{i}^{\kappa}\right)}{i\left(\beta_{i}, w_{i}^{1} A_{i}^{1}\right)+\cdots+i\left(\beta_{i}, w_{i}^{\kappa} A_{i}^{\kappa}\right)},
$$

provided that either the numerator or the denominator of the second term tends to a positive number. Here, for a measured lamination $\lambda$ on $W$, we denote by length $\left(\Psi_{i}(\lambda)\right)$ the sum of the lengths of realisations of the components of $\lambda$ by pleated surfaces in $M_{\Gamma_{i}}$ homotopic to $\Psi_{i}$. Moreover, if $i\left(\alpha_{i}, w_{i}^{1} A_{i}^{1}\right)+\cdots+i\left(\alpha_{i}, w_{i}^{k} A_{i}^{\kappa}\right)$ converges to a positive number, then length $\left(\Psi_{i}\left(\alpha_{i}\right)\right)$ goes to $\infty$. The annuli above can be chosen so that ( $w_{i}^{1} A_{i}^{1} \sqcup \cdots \sqcup w_{i}^{\kappa} A_{i}^{\kappa}$ ) converges to a codimension-1 measured lamination whose support is equal to the Hausdorff limit of $A_{i}^{1} \sqcup \cdots \sqcup A_{i}^{\kappa}$.

Proof By Theorem 3.1 of [50], there is a sequence of essential annuli $A_{i}^{1}, \ldots, A_{i}^{\kappa}$ with weights $w_{i}^{1}, \ldots, w_{i}^{\kappa}$ satisfying the conditions of our lemma except for the last 
sentence. We only need to show that such annuli can be chosen so that they satisfy the last condition.

Let $L$ be a codimension-1 measured lamination in $W$ which is the limit in the measure topology of $w_{i}^{1} A_{i}^{1} \sqcup \cdots \sqcup w_{i}^{\kappa} A_{i}^{\kappa}$. As was shown in the proof of Theorem 3.1 in [50] using the theorem of Morgan and Shalen [41], there is an incompressible branched surface $B$ carrying both $L$ and $A_{i}^{1} \sqcup \cdots \sqcup A_{i}^{\kappa}$ for large $i$, which carries only surface with null Euler characteristic. Consider a weight system $\omega$ on $B$ such that $(B, \omega)$ carries $L$. We approximate $\omega$ by rational weight systems $\omega_{i}$ such that no coordinate of $\omega_{i}$ goes to 0 as $i \rightarrow \infty$. Recall that if $B$ is given a rational weight system, then there is a weighted disjoint union of essential annuli carried by it. (If $B$ with the weight system carries a Möbius band, we consider an annulus lying on the frontier of a twisted regular neighbourhood of the band which doubly covers it. We then give the half of the weight on the Möbius band to the annulus. This does not change the effect of the transverse measure to the simple closed curves on $\partial W$.) If we let $w_{i}^{1} A_{i}^{1} \sqcup \cdots \sqcup w_{i}^{\kappa} A_{i}^{\kappa}$ be weighted annuli carried by $\left(B, \omega_{i}\right)$, then the Hausdorff limit of $A_{i}^{1} \sqcup \cdots \sqcup A_{i}^{\kappa}$ coincide the support of $L$ provided that we let the annulus covering a Möbius band closer and closer to the band as $i \rightarrow \infty$ when we need to consider the Hausdorff limit of Möbius bands.

This implies the following corollary.

Corollary 4.2 Let $\left\{\left(G_{i}, \phi_{i}\right)\right\}$ be a sequence as in Theorem 3.3. If the characteristic compression body $V$ of $C$ is empty (ie $W=C$ ), then $\left\{\left(G_{i}, \phi_{i}\right)\right\}$ converges in $A H(G)$ after passing to a subsequence.

Before starting the proof of Corollary 4.2, we shall consider the general situation when $V$ may not be empty.

Suppose that every subsequence of $\left\{\phi_{i} \mid H^{W}\right\}$ diverges in $A H\left(H^{W}\right)$. Then by the lemma above, we get a sequence of weighted disjoint, nonparallel union of annuli $a_{i}=w_{i}^{1} A_{i}^{1} \sqcup \cdots \sqcup w_{i}^{\kappa} A_{i}^{\kappa}$ describing the divergence. These annuli are disjoint from the torus boundary components of $W$ (passing to a subsequence if necessary) since the translation length of every parabolic element is 0 . By taking a subsequence, we can assume that if we regard $a_{i}$ as a codimension-1 measured lamination in $W$, then it converges to some nonempty codimension-1 measured lamination $\Lambda^{W}$ in $W$ as $i \rightarrow \infty$. We call this measured lamination $\Lambda^{W}$ the limit lamination of $W$. The limit lamination may depend on the choice of a subsequence which was taken in Lemma 4.1. We fix a subsequence in the following argument.

By Jaco-Shalen-Johannson theory [24; 25], all the annuli $A_{i}^{k}$ are properly isotopic into the union of the characteristic pairs $\left(X_{j}, Z_{j}\right)$ of $(W, \partial W)$ each of which is either an 
$I$-pair or a solid torus. Let $\Lambda_{0}$ be a component of $\Lambda^{W}$. Then there is a characteristic pair $\left(X_{0}, Z_{0}\right)$ containing $\Lambda_{0}$ since $\Lambda_{0}$ is approximated by weighted unions of annuli. If $X_{0}$ is an $I$-bundle, we can assume that the annuli approximating $\Lambda_{0}$ are all vertical with respect to the $I$-bundle structure of $X_{0}$. Then $\Lambda_{0}$ is also vertical, and admits an $I$-bundle structure itself whose associated $\partial I$-bundle is $\Lambda_{0} \cap \partial W$. (Refer to Morgan and Shalen [42] for a detailed account of this.) If $X_{0}$ is a solid torus, then $\Lambda_{0}$ itself is either a weighted annulus or a weighted Möbius band. In either case, $\Lambda_{0}$ admits an $I$-bundle structure.

Consider an involution $\iota^{W}$ on $\Lambda^{W} \cap \partial W$ such that for a point $x \in \Lambda^{W} \cap \partial W$, its image $\iota^{W}(x)$ is the other endpoint of the fibre containing $x$ with respect to the $I$-bundle structure obtained as above. Let $\lambda_{0}$ be a component of $\Lambda^{W} \cap \partial W$. Then $\iota^{W}\left(\lambda_{0}\right)$ either coincides with $\lambda_{0}$ or is disjoint from $\lambda_{0}$. When we talk about $\iota^{W}\left(\lambda_{0}\right)$, we regard it as having the transverse measure induced from that of $\lambda_{0}$. Moreover, we have the following lemma.

\section{Lemma 4.3 The following two hold for the involution $\iota^{W}$ defined above.}

(i) If $\iota^{W}\left(\lambda_{0}\right)$ is disjoint from $\lambda_{0}$, then their supports are isotopic in $W$.

(ii) In the case when $\iota^{W}\left(\lambda_{0}\right)=\lambda_{0}$, there are two possibilities:

(a) There is a twisted characteristic $I$-pair over a nonorientable surface $S^{\prime}$ in $W$ such that $\lambda_{0}$ is homotopic in $\partial W$ to a double cover of a measured lamination on the zero-section of $S^{\prime}$ (where we regard $I$ as $[-1,1]$ ). If $\lambda_{0}$ is not a simple closed curve, this is the only possibility.

(b) There is a solid torus component of the characteristic pair in $W$ such that $\lambda_{0}$ is homotopic to a double covering of its core curve.

Proof Let $\Lambda_{0}$ be the component of $\Lambda^{W}$ containing $\lambda_{0}$. As was remarked above, $\Lambda_{0}$ can be assumed to be vertical with respect to the $I$-bundle structure of the characteristic $I$-pair $\left(X_{0}, Z_{0}\right)$ containing $\Lambda_{0}$. Since the union of annuli $A_{i}^{1} \sqcup \cdots \sqcup A_{i}^{\kappa}$ converges to the support of $\Lambda^{W}$ in the Hausdorff topology by the last sentence of Lemma 4.1, there is a subset $a_{i}^{\prime}$ of $A_{i}^{1} \sqcup \cdots \sqcup A_{i}^{\kappa}$, which consists of annuli, converging to the support of $\Lambda_{0}$ in the Hausdorff topology. If $\lambda_{0}$ is disjoint from $\iota^{W}\left(\lambda_{0}\right)$, the $I$-bundle structure of $\Lambda_{0}$ must be trivial. This means that the supports of $\lambda_{0}$ and $\iota^{W}\left(\lambda_{0}\right)$ are isotopic by our definition of isotopy between two laminations, and we are done in this case.

We should also note that if $\Lambda_{0}$ is contained in a characteristic pair $\left(X_{0}, Z_{0}\right)$ that is a product $I$-bundle, then the $I$-bundle structure of $\Lambda_{0}$ must be trivial. Therefore, to consider the remaining case when $\lambda_{0}=\iota^{W}\left(\lambda_{0}\right)$, we can assume that $\left(X_{0}, Z_{0}\right)$ is twisted. 
Now, suppose that $\iota^{W}\left(\lambda_{0}\right)=\lambda_{0}$. Then the characteristic pair $\left(X_{0}, Z_{0}\right)$ is either a twisted $I$-bundle or a solid torus. First consider the case when $X_{0}$ is a twisted $I$-bundle. We consider the base surface $S_{0}$ of the $I$-bundle, which is a nonorientable surface, and by identifying $S_{0}$ with the image of its section, we regard $S_{0}$ as embedded in $X_{0}$ horizontally. We consider the multi-curve $a_{i}^{\prime} \cap X_{0}$, which is regarded as a geodesic lamination and is denoted by $\beta_{i}$. Then $\beta_{i}$ converges to a geodesic lamination $\beta_{\infty}$ over which $\left|\Lambda_{0}\right|$ is a twisted $I$-bundle. The bundle must be twisted since we assumed that ${ }_{\iota} W\left(\lambda_{0}\right)=\lambda_{0}$. We can give a transverse measure to $\beta_{\infty}$ by identifying it with $\Lambda_{0} \cap S_{0}$. This is the case corresponding to (a) of (ii).

Next suppose that $X_{0}$ is a solid torus. Then, $\Lambda_{0}$ is either an annulus or a Möbius band. Since $\iota^{W}\left(\lambda_{0}\right)=\lambda_{0}$, the latter is the case. Then obviously $\lambda_{0}$ is homotopic to a double cover over a core curve of the Möbius band.

Now we shall start a proof of Corollary 4.2. Although this was already proved in [45], the proof here will serve as a perspective for our general argument.

Proof of Corollary 4.2 Suppose, seeking a contradiction, that $\left\{\phi_{i}=\phi_{i} \mid H^{W}\right\}$ diverges and set $a_{i}=w_{i}^{1} A_{i}^{1} \sqcup \cdots \sqcup w_{i}^{\kappa} A_{i}^{\kappa}$ for weighted annuli given in Lemma 4.1. Then $\left\{a_{i}\right\}$ converges to the limit lamination $\Lambda^{W}$ in the measure topology. Let $\lambda$ be $\Lambda^{W} \cap \partial C$, which is disjoint from $T$ as was seen above. Suppose first that one of the laminations $\mu_{j}$ given in the assumption of Theorem 1.2 intersects $\lambda$ essentially. Then, $i\left(\mu_{j}, a_{i}\right)$ converges to a positive number as $i \rightarrow \infty$ passing to a subsequence. It follows that the length of $\Phi_{i}\left(\mu_{j}\right)$ in $M_{\phi_{i}(G)}$ goes to infinity by Lemma 4.1. On the other hand, since the length of $\mu_{j}$ with respect to $v_{i}$ is bounded, by Sullivan's theorem (see Epstein and Marden [20]) or a generalised version of Bers' inequality (see Lemma 2.1 in Ohshika [45]), we see that the length of $\Phi_{i}\left(\mu_{j}\right)$ (on a pleated surface realising it) is also bounded. This is a contradiction.

The same argument applies when $\lambda$ intersects a core curve of a component of $P$ essentially. Therefore we can assume that each component of $\lambda$ is contained in one of $\Sigma_{1}, \ldots, \Sigma_{m}$, say $\Sigma_{j}$. Suppose that $j \leq n$ first. There is a simple closed curve $c$ on $\Sigma_{j}$ intersecting $\lambda$ essentially. It follows that the length of $\Phi_{i}(c)$ goes to infinity by Lemma 4.1. This contradicts (using Sullivan's theorem again) the assumption that the marked conformal structures on $\Sigma_{j}$ converge to $m_{j}$, which implies that the length of every simple closed curve is bounded as $i \rightarrow \infty$.

Therefore $\lambda$ is contained in $\Sigma_{n+1} \sqcup \cdots \sqcup \Sigma_{m}$. Since $\lambda$ cannot intersect $\mu_{j}$ essentially and $\mu_{j}$ is arational in $\Sigma_{j}$, the support of a component of $\lambda$ coincides with that of some $\mu_{j}$. This implies that each component $\lambda_{0}$ of $\lambda$ is arational in some $\Sigma_{j}$; hence in particular, $\Sigma_{j}$ is the minimal supporting surface of $\lambda_{0}$. Suppose that $\lambda_{0}$ is a component 
of $\lambda$ such that $\iota^{W}\left(\lambda_{0}\right)$ is disjoint from $\lambda_{0}$. Then $\lambda_{0}$ is isotopic to $\iota^{W}\left(\lambda_{0}\right)$. This implies that the minimal supporting surfaces $T\left(\lambda_{0}\right)$ and $T\left(\iota^{W}\left(\lambda_{0}\right)\right)$, both of which are among $\Sigma_{n+1}, \ldots, \Sigma_{m}$, are homotopic (by (ii) of Lemma 2.3), and cobound relative to $P$ a product $I$-bundle since no two distinct components of $P$ are homotopic. This is an excluded case in Theorem 1.2.

Suppose next that $\iota^{W}\left(\lambda_{0}\right)=\lambda_{0}$. Again the minimal supporting surface of $\lambda_{0}$ is some $\Sigma_{j}$. Then, there is a twisted $I$-bundle bounded relative to $P$ by $\Sigma_{j}$, and $\lambda_{0}$ doubly covers a lamination on the zero-section by Lemma 4.3, as was observed above. This is also an excluded case in Theorem 1.2.

Thus we have shown that $\left\{\left(G_{i}, \phi_{i}\right)\right\}$ converges in $A H(G)$ in this situation.

From now on until the end of the proof of Theorem 3.3, we assume that $V$ is not empty. Consider two distinct components $W, W^{\prime}$ of the irreducible part for which both $\left\{\phi_{i} \mid H^{W}\right\}$ and $\left\{\phi_{i} \mid H^{W^{\prime}}\right\}$ diverge in $A H\left(H^{W}\right)$ and $A H\left(H^{W^{\prime}}\right)$ respectively, supposing that there are such components. Let $\Lambda^{W}$ and $\Lambda^{W^{\prime}}$ be the limit laminations of $W$ and $W^{\prime}$ respectively. We say that $W$ dominates $W^{\prime}$ if for closed curves $\gamma$ on $\partial W$ intersecting $\Lambda^{W}$ essentially and $\gamma^{\prime}$ on $\partial W^{\prime}$ intersecting $\Lambda^{W^{\prime}}$ essentially, we have

$$
\lim _{i \rightarrow \infty} \frac{\text { length } \phi_{i}\left(\gamma^{\prime}\right)}{\text { length } \phi_{i}(\gamma)}=0,
$$

where length denotes the translation length. Obviously this definition does not depend on the choice of $\gamma$ and $\gamma^{\prime}$. By taking a subsequence, we can assume that for any two components $W, W^{\prime}$ of the irreducible parts unless one of them dominates the other,

$$
\lim _{i \rightarrow \infty} \frac{\text { length } \phi_{i}\left(\gamma^{\prime}\right)}{\text { length } \phi_{i}(\gamma)}
$$

exists and is a positive number. It also follows from the definition that the relation of domination is transitive: if $W$ dominates $W^{\prime}$ and $W^{\prime}$ dominates $W^{\prime \prime}$, then $W$ dominates $W^{\prime \prime}$. Therefore we can regard this relation as ordering, and denote $W^{\prime} \prec_{\text {dom }} W$ if $W$ dominates $W^{\prime}$.

Let $\mathcal{B}$ be the set of all components of the irreducible part that are maximal with respect to the ordering $\prec_{\text {dom }}$. Then by definition, no component contained in $\mathcal{B}$ is dominated by another component. Also, by a remark in the previous paragraph, if both $W$ and $W^{\prime}$ are contained in $\mathcal{B}$, then

$$
\lim _{i \rightarrow \infty} \frac{\text { length } \phi_{i}\left(\gamma^{\prime}\right)}{\text { length } \phi_{i}(\gamma)}
$$

is a positive number, and if $W \in \mathcal{B}$ and $W^{\prime} \notin \mathcal{B}$ then $W$ dominates $W^{\prime}$. We see that $\mathcal{B}$ is nonempty if $\left\{\phi_{i} \mid H^{W}\right\}$ diverges for some component $W$ of the boundary-irreducible 
part. We call components contained in $\mathcal{B}$ the dominating components. We define $\mathcal{B}$ to be empty if there is no component $W$ of the boundary-irreducible part such that $\left\{\phi_{i} \mid H^{W}\right\}$ diverges.

\section{Convergence of function groups}

Take a compressible boundary component $S_{k}$ of $C$. We denote by $V_{k}$ the component of the characteristic compression body $V$ of $C$ which contains $S_{k}$ as its exterior boundary. We consider a subgroup $H^{k}$ of $G$ associated to the image of $\pi_{1}\left(S_{k}\right)$ in $\pi_{1}(C) \cong G$ by a homomorphism induced by the inclusion. The image of $\pi_{1}\left(S_{k}\right)$ can be identified with $\pi_{1}\left(V_{k}\right)$.

Recall that we denote the union of core curves of $P$ on $S_{k}$ by $C^{k}$ and the union of measured laminations $\mu_{j}^{k}$ on $S_{k}$ by $M^{k}$. The compression body $V_{k}$ may have interior boundary components contained in $\partial C$. In this case we add core curves of $P$ and the $\mu_{j}$ on these components to $C^{k}$ and $M^{k}$ respectively. We denote the unions by $\bar{C}^{k}$ and $\bar{M}^{k}$. If $\partial_{i} V_{k} \cap \partial C=\varnothing$, then we set $\bar{C}^{k}=C^{k}$ and $\bar{M}^{k}=M^{k}$.

Lemma 5.1 Suppose that $\left\{\phi_{i}\right\}$ diverges in $A H(G)$ even after passing to a subsequence. Then one of the following two conditions holds:

(i) $\mathcal{B}$ is empty, or

(ii) there are a dominating component $W$ with its limit lamination $\Lambda^{W}$, a component $V_{k}$ of $V$ meeting $W$, and a component of $\Lambda^{W} \cap \partial_{i} V_{k}$ whose support is not isotopic in $V_{k}$ to the support of any component of $C^{k} \cup M^{k}$.

Proof We assume that there is at least one dominating component, and shall show that the second alternative holds. If $\Lambda^{W}$ is disjoint from $V$ for some dominating component $W$ (ie the boundary of $\Lambda^{W}$ lies in $(\partial C \backslash V)$ ), then we can argue entirely in the same way as the proof of Corollary 4.2 and get a contradiction. Therefore, we can assume that $\Lambda^{W} \cap V \neq \varnothing$ for every dominating component $W$.

Let $V_{j}$ be a component of $V$ intersecting some dominating component. Suppose that for every dominating component $W$ intersecting $V_{j}$, each component $\lambda$ of the lamination $\Lambda^{W} \cap \partial_{i} V_{j}$ has support isotopic in $V_{j}$ to the support of a component $\lambda^{\prime}$ of $C^{j} \cup M^{j}$. (Otherwise, we have only to set $V_{k}$ to be $V_{j}$ and get the second alternative.)

Take a component $\lambda$ of $\Lambda^{W} \cap \partial_{i} V_{j}$ for a dominating component $W$ intersecting $V_{j}$. We consider the lamination $\iota^{W}(\lambda)$. As was observed in the previous section, either $\iota^{W}(\lambda)=\lambda$ or $\iota^{W}(\lambda)$ is disjoint from $\lambda$. 
(a) Let us first consider the case when $\iota^{W}(\lambda)=\lambda$.

Recall that we assumed that there is a component $\lambda^{\prime}$ of $C^{j} \cup M^{j}$ lying on $S_{j}$ whose support is isotopic in $V_{j}$ to that of $\lambda$. Suppose first that $\lambda^{\prime}$ is contained in $C^{j}$. Then $\lambda$ is a simple closed curve, hence is homotopic to a double covering of either a simple closed curve on the base surface of a twisted $I$-bundle or a core curve of a solid torus, either of which is embedded in $W$, as was observed in Lemma 4.3. This is a contradiction because every component of $C^{j}$ is a core curve of a paring locus $P$, hence represents a primitive class of $\pi_{1}(C)$.

Suppose next that $\lambda^{\prime}$ is contained in $M^{j}$. Again by Lemma 4.3, there is an embedded twisted $I$-bundle $X$ in $W$ and $\lambda$ is homotopic to a double cover of a measured lamination on the zero-section of the base surface. By assumption, $\lambda^{\prime}$ is arational on a component $\Sigma_{u}$ of $S_{j} \backslash C^{j}$ and is contained in the Masur domain of $\Sigma_{u}$. Let $T(\lambda)$ be the minimal supporting surface of $\lambda$ on $\partial_{i} V_{j}$. Then $T(\lambda)$ doubly covers a subsurface $T^{\prime}$ on the zero-section of the base surface of $X$. Since $\lambda$ is isotopic to $\lambda^{\prime}$, and neither $\partial T(\lambda)$ lying on $\partial_{i} V_{j}$ nor $\partial \Sigma_{u}$ contains a meridian, the surface $T(\lambda)$ is homotopic to $\Sigma_{u}$ in $V_{j}$ by Lemma 2.3 .

Since $T(\lambda)$ doubly covers nonorientable $T^{\prime}$ and every boundary component of $T^{\prime}$ is an orientation-preserving curve on $T^{\prime}$, each boundary component of $T(\lambda)$ is one of the two components of the preimage of a boundary component of $T^{\prime}$, hence is homotopic in $W$ to another boundary component of $T(\lambda)$. Therefore, each component of $c$ of $\partial \Sigma_{u}$ is homotopic in $V_{j} \cup W$ to another boundary component of $\partial \Sigma_{u}$. (The boundary of $\Sigma_{u}$ cannot be empty since $S_{j}$ cannot be homotopic to a surface covered by a subsurface of a component of $\partial_{i} V_{j}$.) Since the boundary components of $\Sigma_{u}$ are all in $C^{j}$, which are core curves of the paring locus $P$, two of them are homotopic in $C$ only when they are parallel on $\partial V_{j}$. If this happens, then the entire $S_{j}$ is obtained by pasting annuli in $P$ to $\Sigma_{u}$, and $V_{j} \cup X$ itself is a twisted $I$-bundle. This is impossible since $V_{j}$ is a compression body.

(b) Next we consider the case when $\iota^{W}(\lambda)$ is disjoint from $\lambda$.

If $\iota^{W}(\lambda)$ is also contained in $\partial_{i} V_{j}$, then $\iota^{W}(\lambda)$ is also isotopic in $V_{j}$ to a component of $C^{j} \cup M^{j}$, and by an argument similar to the above, we are lead to a contradiction with either the assumption that $C^{j}$ consists of core curves of the paring locus $P$ and the fact that $V_{j}$ cannot be contained in a product $I$-bundle if $W$ is not empty.

Suppose now that $\iota^{W}(\lambda)$ lies on $\partial W \backslash V$, hence on an incompressible component of $\partial C$. If either one of the $\mu_{j}$ or a core curve in $P$ intersects $\iota^{W}(\lambda)$ essentially, then we get a contradiction as in the proof of Corollary 4.2. Therefore, $\iota^{W}(\lambda)$ is contained in a component $\Sigma$ of $\partial C \backslash P$, and by the same argument as in Corollary 4.2, we see 
that it is arational there (ie $T\left(\iota^{W}(\lambda)\right)=\Sigma$ ). Moreover, since $\Sigma$ lies on $\partial W$, it is incompressible and $\iota^{W}(\lambda)$ is contained in the Masur domain of $\Sigma$, which is the entire measured lamination space. Then $\Sigma$ and $\Sigma_{u}$ are homotopic in $C$ by Lemma 2.3. If $\Sigma$ and $\Sigma_{u}$ have nonempty boundaries, their boundaries, which lie in distinct components of $\partial C$, consist of (simple closed curves homotopic to) core curves of $P$. This is a contradiction since no two distinct components of $P$ are homotopic in $C$. If $\Sigma$ and $\Sigma_{u}$ are closed surfaces, then $W$ itself is a product $I$-bundle, which contradicts the definition of the characteristic compression body.

Suppose next that $\iota^{W}(\lambda)$ is contained in another component $V_{k}$ of $V$ with $k \neq j$. If $\iota^{W}(\lambda)$ is not isotopic in $V_{k}$ to a component of $C^{k} \cup M^{k}$, then this $V_{k}$ is what we were looking for to get condition (ii). Suppose, on the contrary, that $\iota^{W}(\lambda)$ is isotopic in $V_{k}$ to a component $\lambda^{\prime \prime}$ of $C^{k} \cup M^{k}$. Then $\lambda^{\prime}$ and $\lambda^{\prime \prime}$ are isotopic in $C$. If $\lambda^{\prime \prime}$ is a simple closed curve, ie, contained in $C^{k}$, then this means that there are core curves of distinct two components of $P$ which are homotopic in $C$. This is a contradiction. Suppose that $\lambda^{\prime \prime}$ is contained in $M^{k}$. Then $\lambda^{\prime \prime}$ is arational in some component $\Sigma_{v}$ of $S_{k} \backslash C^{k}$ and $\Sigma_{v}$ is homotopic to the minimal supporting surface of $\iota^{W}(\lambda)$. (Since no boundary component of $\Sigma_{v}$ or $T\left(\iota^{W}(\lambda)\right)$ is a meridian, we can use Lemma 2.3.) On the other hand, $\lambda^{\prime}$ is arational in a component $\Sigma_{u}$ of $S_{j} \backslash C^{j}$ which is homotopic to the minimal supporting surface $T(\lambda)$. Since $T(\lambda)$ is homotopic to $T\left(\iota^{W}(\lambda)\right)$, this implies that $\Sigma_{u}$ is homotopic to $\Sigma_{v}$. In particular, a boundary component of $\Sigma_{u}$, which is contained in $C^{j}$, is homotopic to a boundary component of $\Sigma_{v}$, which is contained in $C^{k}$. (If $\Sigma_{u}$ is closed, we get a contradiction as before.) This contradicts the fact that no two core curves of distinct components of $P$ are homotopic. Thus we have completed the proof.

Now we start the proof of Theorem 3.3. We shall first show that for each component $V_{k}$ of $V$, the sequence $\left\{\left(\phi_{i}\left(H^{k}\right), \phi_{i} \mid H^{k}\right) \in A H\left(H^{k}\right)\right\}$ converges if $\mathcal{B}$ is empty, using Lecuire's result on measured laminations in $\mathcal{D}$. For that, we need to prove that we can extend $\bar{C}^{k} \cup \bar{M}^{k}$ to a measured lamination contained in $\mathcal{D}\left(V_{k}\right)$.

Lemma 5.2 Take an arational measured lamination on each component of the complement $\left(V_{k} \cap \partial C\right) \backslash P$ among $\Sigma_{1}, \ldots, \Sigma_{n}$, which is contained in its Masur domain and does not have support isotopic in $V_{k}$ to the support of a component of $M^{k}$ in such a way that no two of them have supports isotopic to each other in $V_{k}$. (Recall that $\Sigma_{1}, \ldots, \Sigma_{n}$ are components of $\partial C \backslash P$ where the marked conformal structures converge in the Teichmüller spaces.) Denote the union of all of these arational measured laminations by $L^{k}$. Take also an arational measured lamination on each interior boundary component of $V_{k}$ that is not contained in $\partial C$, and let $Q^{k}$ be their union. Then $\bar{C}^{k} \cup \bar{M}^{k} \cup L^{k} \cup Q^{k}$ is contained in $\mathcal{D}\left(V_{k}\right)$. 
Proof By the definition of $\mathcal{D}$, we have only to show that there is $\eta>0$ such that every meridian and the boundary of every essential annulus has intersection number greater than $\eta$ with $\bar{C}^{k} \cup \bar{M}^{k} \cup L^{k} \cup Q^{k}$. Suppose, seeking a contradiction, that there is a sequence of meridians $\left\{\partial D_{l}\right\}$ or of boundaries of essential annuli $\left\{\partial A_{l}\right\}$ such that $i\left(\partial D_{l}, \bar{C}^{k} \cup \bar{M}^{k} \cup L^{k} \cup Q^{k}\right)$ or $i\left(\partial A_{l}, \bar{C}^{k} \cup \bar{M}^{k} \cup L^{k} \cup Q^{k}\right)$ goes to 0 as $l \rightarrow \infty$. Then after taking a subsequence, we can assume that $\partial D_{l}$ or $\partial A_{l}$ is disjoint from $\bar{C}^{k}$ for every $l$, for otherwise it has intersection number at least 1 with $\bar{C}^{k}$. For meridians, we set $c_{l}=\partial D_{l}$, and for annuli, we set $c_{l}$ to be one of the components of $\partial A_{l}$. Then, passing to a subsequence, all of the $c_{l}$ can be assumed to be contained in a component $\Sigma$ of $\partial V_{k} \backslash \bar{C}^{k}$ since they are disjoint from $\bar{C}^{k}$ and there are only finitely many components of $\partial V_{k} \backslash \bar{C}^{k}$. We can also assume that the same holds even if we choose the other boundary component of $A_{l}$ for each $l$. Since $\mu_{j}$ or a component of $L^{k} \cup Q^{k}$ given on $\Sigma$ is arational, it follows that its support coincides with a minimal component $\ell$ of the Hausdorff limit of the $c_{l}$, for both choices of the boundary components of $A_{l}$. If the $c_{l}$ are meridians (of $\Sigma$ ), their Hausdorff limit contains a homoclinic leaf. (Refer to Théorème 1.8 of Otal [52].) By Lemma 2.1, this contradicts the assumptions that any $\mu_{j}$ or any component of $L^{k}$ is contained in the Masur domain, and that $Q^{k}$ lies on the interior boundary of $V_{k}$.

Next suppose that the $c_{l}$ are boundary components of the $A_{l}$. We consider first the case when the two boundary components of $A_{l}$ are contained in distinct components $\Sigma$ and $\Sigma^{\prime}$ of $\partial V_{k} \backslash \bar{C}^{k}$. Let $\ell, \ell^{\prime}$ be the Hausdorff limits of $A_{l} \cap \Sigma$ and $A_{l} \cap \Sigma^{\prime}$, which are isotopic in $V_{k}$ by definition. Since the Hausdorff limit of $\partial A_{l}$ does not intersect $\bar{M}^{k} \cup L^{k} \cup Q^{k}$ essentially, both $\ell$ and $\ell^{\prime}$ consist of a unique arational minimal component and isolated noncompact leaves spiralling around them. Their minimal supporting surfaces are $\Sigma$ and $\Sigma^{\prime}$ respectively. Also, the minimal components of both $\ell$ and $\ell^{\prime}$ carry transverse measures which give rise to measured laminations in the Masur domains of $\Sigma$ and $\Sigma^{\prime}$ since they coincide with the supports of components of $\bar{M}^{k} \cup Q^{k} \cup L^{k}$. Since their boundaries do not contain meridians, by Lemma 2.3, $\Sigma$ and $\Sigma^{\prime}$ are homotopic. Moreover, since no two distinct components of $\bar{C}^{k}$ are homotopic and no interior boundary component is homotopic to another boundary component, this is possible only when $\Sigma$ and $\Sigma^{\prime}$ lie on $\partial V_{k} \cap \partial C$, all the boundary components of $\Sigma$ are homotopic in $\partial V_{k}$ to boundary components of $\Sigma^{\prime}$, and the same holds interchanging $\Sigma$ with $\Sigma^{\prime}$. This happens only when $V_{k}$ is a handlebody and homeomorphic to a product $I$-bundle over a compact surface as a pared manifold. This in particular implies that the boundary-irreducible part is empty; hence $V_{k}=C$, and $\Sigma, \Sigma^{\prime}$ can be assumed to be $\Sigma_{1}, \Sigma_{2}$.

By the assumption of Theorem 1.2, if the $\mu_{j}$ are given on both of $\Sigma_{1}$ and $\Sigma_{2}$, ie $n=0$, then the supports of $\mu_{1}$ and $\mu_{2}$ are not isotopic. This contradicts the fact that $\ell$ and $\ell^{\prime}$ 
are isotopic and the supports of $\mu_{1}, \mu_{2}$ are their minimal components. If $\mu_{j}$ is not given on $\Sigma_{1}$, ie, $n \geq 1$, then by our assumption, the component $\lambda_{1}$ of $L^{k}$ on $\Sigma_{1}$ has support which is not isotopic to that of $\mu_{2}$ or $\lambda_{2}$. This again contradicts the fact that $\ell$ and $\ell^{\prime}$ are isotopic.

Next we consider the case when both of the boundary components of $A_{l}$ lie in the same component $\Sigma$ of $\partial V_{k} \backslash \bar{C}^{k}$. We need to divide our argument into two subcases depending on whether $\Sigma$ is compressible or not. Suppose first that $\Sigma$ is compressible. Let $D$ be a compressing disc of $\Sigma$. Then, since $\left\{\partial A_{l}\right\}$ converges to an arational lamination, $A_{l}$ intersects $D$ essentially for large $l$. Therefore, we can either boundary-compress $A_{l}$ along an outermost semidisc on $D$ bounded by $A_{l} \cap D$, or get a compressing disc intersecting $A_{l}$ along fewer arcs than $D$ which is obtained by cutting $D$ along an arc cobounding with $\partial A_{l}$ an outermost semidisc on $A_{l}$. In the former case, we get a meridian $d_{l}$ of $\Sigma$ which is disjoint from $A_{l}$ by boundary-compressing $A_{l}$. Also in the latter case, by repeating the same operation, we eventually get a meridian $d_{l}$ of $\Sigma$ which is disjoint from $A_{l}$. Then the Hausdorff limit $d^{\prime}$ of the $d_{l}$ does not intersect the Hausdorff limit of $\partial A_{l}$ transversely, hence neither does it a component of $\bar{M}^{k} \cup L^{k} \cup Q^{k}$ on $\Sigma$, which is an arational lamination. Then by the same argument using the homoclinicity as for the Hausdorff limit of $\left\{D_{l}\right\}$ above, we get a contradiction.

Suppose next that $\Sigma$ is incompressible. Then by applying the Jaco-Shalen-Johannson theory to $\left(V_{k}, \Sigma\right)$, we see that there is a characteristic pair $X$ which is a union of $I$-bundles and solid tori intersecting $\partial V_{k}$ in $\Sigma$, into which every essential annulus with boundary on $\Sigma$ can be properly homotoped. Since $\left\{\partial A_{l}\right\}$ converges to an arational lamination, there is an $I$-bundle component $X_{0}$ of $X$ into which $A_{l}$ can be properly homotoped for large $l$, and $\Sigma$ is isotopic in $V_{k}$ to the associated $\partial I$-bundle of $X_{0}$. This is possible only when $X_{0}$ is a twisted $I$-bundle. We also see that $V_{k}=X_{0}$ since no two components of $\partial \Sigma$ not homotopic on $\partial V_{k}$ are homotopic in $V_{k}$. This implies that $V_{k}$ is a twisted $I$-bundle over a nonorientable surface, and contradicts the fact that $V_{k}$ is a compression body.

We need to use the following lemma, which was proved by Lecuire in [28].

Lemma 5.3 Let $V$ be a compression body and $S$ its exterior boundary. Suppose that $\pi_{1}(V)$ acts on an $\mathbb{R}$-tree $\mathcal{T}$ by isometries with small edge-stabilisers. Let $\mu$ be a measured lamination contained in $\mathcal{D}(V)$. Then there exists a $\pi_{1}(S)$-equivariant map $F: \mathbb{H}^{2} \rightarrow \mathcal{T}$ which realises at least one component of $\mu$. (Here we regard $\pi_{1}(S)$ as acting on $\mathcal{T}$ by pre-composing the epimorphism from $\pi_{1}(S)$ to $\pi_{1}(V)$ induced by the inclusion.) 
As a first step of the proof of Theorem 3.3, we shall show the following proposition. This is a case which Lecuire's Theorem 6.6 in [28] already covers. Still, we shall give an outline of proof here based on Lecuire's lemma above so that we can refer to it in the argument for the next case when $\mathcal{B}$ is not empty.

Proposition 5.4 Suppose that $\mathcal{B}$ is empty. Then for every component $V_{k}$ of $V$, the quasiconformal deformations of $H^{k}$ given by the $\phi_{i} \mid H^{k}$ converge after taking a subsequence and conjugates.

Proof Suppose, seeking a contradiction, that $\left\{\phi_{i} \mid H^{k}\right\}$ does not have a convergent subsequence in $A H\left(H^{k}\right)$. Then, by the work of Morgan and Shalen [41], Bestvina [5] and Paulin [54], there is an isometric action with small edge-stabilisers of $H^{k}$ on an $\mathbb{R}$-tree $\mathcal{T}$ which is a Gromov limit of the rescaled action of $\phi_{i}\left(H^{k}\right)$ on $\epsilon_{i} \mathbb{H}^{3}$ with $\epsilon_{i} \rightarrow 0$. This can be regarded as an action of $\pi_{1}\left(V_{k}\right)$ on $\mathcal{T}$. By Lemmata 5.2 and 5.3, one of the components of $\bar{C}^{k} \cup \bar{M}^{k} \cup L^{k} \cup Q^{k}$, which we shall denote by $\chi$, is realised in $\mathcal{T}$.

Suppose first that $\chi$ is a component of $\bar{C}^{k}$. Then there is an element $\gamma \in H^{k}$ whose conjugacy class is represented by $\chi$. Since $\chi$ is realised in $\mathcal{T}$, the translation length of $\phi_{i}(\gamma)$ goes to infinity as $i \rightarrow \infty$. On the other hand, by our construction, the length of $\chi$ with respect to the conformal structure at infinity $v_{i}$ goes to 0 . This implies, as was shown by Sugawa [57, Theorem 6.2] (or by the main theorem of Canary [18]), that the translation length of $\phi_{i}(\gamma)$ also goes to 0 . Thus we are lead to a contradiction.

Next consider the case when $\chi$ is a component $\mu$ of $\bar{M}^{k}$. By Lemma 3.2, there is a sequence of weighted simple closed curves $\left\{r_{n} c_{n}\right\}$ on a component of $S_{k} \backslash C^{k}$ such that $r_{n} c_{n}$ converges to $\mu$ and length $v_{i}\left(r_{i} c_{i}\right)$ goes to 0 as $i \rightarrow 0$. We should also note that for any meridian $m$ in $\Sigma$, its length with respect to $v_{i}$ goes to $\infty$ since $i(m, \mu)>0$. Therefore, by the main theorem of Canary [15], $r_{i}$ length $\left(\phi_{i}\left(c_{i}\right)\right)$ in $M_{\phi_{i}}\left(H^{k}\right)$ goes to 0 , where length denotes the translation length. Since $\chi$ is arational, the Hausdorff limit of $c_{n}$, which we denote by $\xi$, is the union of $\chi$ and finitely many isolated noncompact leaves. Since $\chi$ is realised in $\mathcal{T}$, by the same argument as Théorème 3.1.4 of Otal [53], we see that $\xi$ is also realised in $\mathcal{T}$. Furthermore, Otal's argument implies that there is a train track carrying $\xi$ which is realised in $\mathcal{T}$. This implies that $r_{i}$ length $\left(\phi_{i}\left(c_{i}\right)\right)$ in $M_{\phi_{i}\left(H^{k}\right)}$ must go to infinity as $i \rightarrow \infty$ since $\tau$ is mapped to a train track with geodesic branches and small exterior angles at switches in $M_{\phi_{i}\left(H^{k}\right)}$ (see Chapitre 3 of [53]). This is a contradiction.

Next we consider the case when $\chi$ is a component of $L^{k}$. Then $\chi$ lies on $\Sigma_{j}$ on which the conformal structure converges to $m_{j}$. Therefore, the length with respect 
to $n_{i}^{k}$ of $r_{i} c_{i}$ taken as before is bounded as $i \rightarrow \infty$. Thus, by the same argument as the previous case, we get a contradiction.

Finally, we consider the case when $\chi$ is a component of $Q^{k}$. Let $\Sigma$ be the interior boundary component not contained in $\partial C$ on which $\chi$ lies. There is a component $W$ of the boundary-irreducible part containing $\Sigma$ as a boundary component. Since we assumed that $\mathcal{B}$ is empty, $\phi_{i} \mid H^{W}$ converges, hence in particular, the length of $\Phi_{i}(\chi)$ in $M_{\phi_{i}(G)}$ (hence also that in $M_{\phi_{i}\left(H^{k}\right)}$ ) is also bounded. This contradicts the fact that $\chi$ is realised in $\mathcal{T}$ by the same argument as above.

Now we consider the case when $\mathcal{B}$ is not empty. By Lemma 5.1, there are a dominating component $W$ and a component $V_{k}$ of $V$ such that there is a component of $\Lambda^{W} \cap \partial_{i} V_{k}$ whose support is not isotopic in $V_{k}$ to the support of a component of $\bar{C}^{k} \cup \bar{M}^{k}$. We shall show that this will lead to a contradiction.

Proposition 5.5 In the settings of Theorem 3.3, if $V$ is not empty, then $\mathcal{B}$ must be empty.

Proof To prove this proposition, we need to analyse an action on an $\mathbb{R}$-tree in the same way as the proof of Lemma 5.3 in [28].

Suppose, seeking a contradiction, that neither $V$ nor $\mathcal{B}$ is empty. Then there is a component $V_{k}$ of $V$ intersecting $\Lambda^{W}$ as we mentioned just before the proposition. Then $\left\{\phi_{i} \mid H^{k}\right\}$ must diverge in $A H\left(H^{k}\right)$ since $\Lambda^{W}$ intersects $\partial_{i} V_{k}$. By the same argument as Proposition 5.4, there is a limit isometric action $\rho$ of $H^{k} \cong \pi_{1}\left(V_{k}\right)$ on an $\mathbb{R}$-tree $\mathcal{T}$ having small edge-stabilisers. We first consider a special case when the restriction of $\rho$ to every interior boundary component of $V_{k}$ has a global fixed point in $\mathcal{T}$. In this case, we can argue as in the proof of Proposition 5.4. We extend $\bar{C}^{k} \cup \bar{M}^{k}$ by adding $L^{k}$ and $Q^{k}$ using Lemma 5.2. By Lemma 5.3, one of the components of $\bar{C}^{k} \cup \bar{M}^{k} \cup L^{k} \cup Q^{k}$ is realised by $\rho$. By the same argument as the proof of Proposition 5.4, we see that it is impossible that a component of $\bar{C}^{k} \cup \bar{M}^{k} \cup L^{k}$ is realised by $\rho$. If a component $\chi$ of $Q^{k}$ is realised by $\rho$, then we consider the component $\Sigma$ of $\partial_{i} V_{k}$ on which $\chi$ lies. Then the restriction of $\rho$ to $\pi_{1}(\Sigma)$ is nontrivial. This contradicts the assumption of our special case here.

Now, we assume until the end of the proof that there is at least one component of $\partial_{i} V_{k}$ on which the restriction of $\rho$ is nontrivial, ie, does not have a global fixed point. Regard $\rho$ as an action of $\pi_{1}\left(S_{k}\right)$ (recall that $S_{k}=\partial_{e} V_{k}$ ) by pre-composing the epimorphism from $\pi_{1}\left(S_{k}\right)$ to $\pi_{1}\left(V_{k}\right)$ induced by the inclusion.

Take a sequence of weighted multicurves $\left\{c_{n}\right\}$ decomposing $S_{k}$ into pairs of pants converging to $C^{k} \cup M^{k} \cup\left(L^{k} \cap S_{k}\right)$ in $\mathcal{M L}\left(S_{k}\right)$ as $n \rightarrow \infty$. Since $C^{k} \cup M^{k} \cup\left(L^{k} \cap S_{k}\right)$ 
is maximal, by approximating it by a train track with complementary regions whose vertices correspond one-to-one to ideal vertices of the complementary regions of $C^{k} \cup M^{k} \cup\left(L^{k} \cap S_{k}\right)$ and giving rational weights, we can assume that a union of some components of $\left|c_{n}\right|$ (the support of $c_{n}$ ) converges to the support of $C^{k} \cup M^{k} \cup\left(L^{k} \cap S_{k}\right)$ with respect to the Hausdorff topology. As was shown by Morgan and Otal [40], there are an action $\rho_{n}$ of $\pi_{1}\left(S_{k}\right)$ on an $\mathbb{R}$-tree $\mathcal{T}_{n}$, which is dual to a measured lamination $\zeta_{n}$ on $S_{k}$, and a morphism $\pi_{n}$ from $\mathcal{T}_{n}$ to $\mathcal{T}$ such that the action obtained by pushing $\rho$ forward by $\pi_{n}$, which we denote by $\left(\pi_{n}\right)_{*} \rho_{n}$, coincides with $\rho$, and the translation length of $c_{n}$, ie the weighted sum of the translation lengths of the components of $c_{n}$, with respect to $\rho_{n}$ is equal to that with respect to $\rho$. Now we consider the Hausdorff limit $\zeta_{\infty}$ of $\left\{\left|\zeta_{n}\right|\right\}$. As was shown in the proof of Theorem 3 of Kleineidam and Souto [27], if a component of $C^{k} \cup M^{k} \cup\left(L^{k} \cap S_{k}\right)$ intersects $\zeta_{\infty}$ essentially, then it is realised in $\mathcal{T}$. This is a contradiction as in the proof of Proposition 5.4.

Therefore, $\zeta_{\infty}$ does not intersect $C^{k} \cup M^{k} \cup\left(L^{k} \cap S_{k}\right)$ essentially. We consider the measured lamination $\Lambda^{W} \cap \partial_{i} V_{k}$ for all the components $W \in \mathcal{B}$, and let $\hat{v}$ be the union of all such laminations on $\partial_{i} V_{k}$. By Lemma 5.1, there are a component $F$ of $\partial_{i} V_{k}$ and a component $v_{0}$ of $\hat{v}$ having support which is not isotopic in $V_{k}$ to the support of a component of $C^{k} \cup M^{k}$. Moreover, we see that no component of $\hat{v}$ is isotopic in $V_{k}$ to a component of $L^{k} \cap S_{k}$ as follows.

Let $\lambda_{j}$ be a component of $L^{k} \cap S_{k}$, and suppose that $\lambda_{j}$ lies on $\Sigma_{j}$ which is among $\Sigma_{1}, \ldots, \Sigma_{n}$. Then we can take a simple closed curve $c$ on $\Sigma_{j}$ intersecting $\lambda_{j}$ essentially. Suppose, seeking a contradiction, that $\lambda_{j}$ is isotopic in $V_{k}$ to a component $v_{0}$ of $\widehat{v}$. Then the minimal supporting surface $T\left(v_{0}\right)$ is homotopic to $\Sigma_{j}$ in $V_{k}$ by Lemma 2.3. This implies that there is a simple closed curve $c^{\prime}$ on $T\left(v_{0}\right)$ homotopic to $c$ intersecting $v_{0}$ essentially. In particular, the translation length of $\phi_{i}\left(c^{\prime}\right)$ goes to infinity as $i \rightarrow \infty$. By using the fact that the length of $c$ with respect to $v_{j}$ is bounded, and either Theorem 6.2 in Sugawa [57] or the main theorem of Canary [18], we get a contradiction.

Recall that we assumed that for some component of $\partial_{i} V_{k}$, the restriction of $\rho$ to the component is nontrivial. Consider the component $F$ of $\partial_{i} V_{k}$ containing $v_{0}$ as defined above. Since all the $W$ in $\mathcal{B}$ are dominating and some $\Lambda^{W}$ with $W \in \mathcal{B}$ intersects $F$, if the restriction of $\rho$ to $F$ is trivial, then the restriction of $\rho$ to every component of $\partial_{i} V_{k}$ is trivial. Therefore, under the present assumption, the restriction of $\rho$ to $\pi_{1}(F)$ is a nontrivial action having small edge-stabilisers since $F$ is incompressible. By Skora's theorem [56], it is dual to some measured lamination $v$ on $F$. By the definition of $\Lambda^{W}$, if we consider the restriction of $\phi_{i}$ to the subgroup corresponding to $\pi_{1}(F)$ and its rescaled Gromov limit, we see that $v$ must coincide with $\hat{v} \cap F$ up to a scalar multiple. Since $V_{k}$ is a compression body, there is a surface $\hat{F}$ on $S_{k}$ each of whose 
boundary component is a meridian such that the surface obtained by attaching disjoint compressing discs to $\partial \hat{F}$ is isotopic to $F$ in $V_{k}$. Let $v_{1}$ be a measured lamination on $\widehat{F}$ which is isotopic to $v_{0}$.

Recall that we have actions $\rho_{n}$ of $\pi_{1}\left(S_{k}\right)$ on $\mathbb{R}$-trees $\mathcal{T}_{n}$, which are dual to measured laminations $\zeta_{n}$ on $S_{k}$. As was shown in the proof of Proposition 6.1 in Lecuire [28], we see that $\left|\zeta_{n}\right|$ is constant for large $n$, which implies that $\zeta_{\infty}$ is the support of a measured lamination, which we denote by $\zeta$. As was seen above, $\zeta_{\infty}$ cannot intersect $C^{k} \cup M^{k} \cup\left(L^{k} \cap S_{K}\right)$ essentially; hence we have $i\left(C^{k} \cup M^{k} \cup\left(L^{k} \cap S_{K}\right), \zeta\right)=0$. Since $C^{k} \cup M^{k} \cup\left(L^{k} \cap S_{k}\right)$ is maximal, we see that the support of $\zeta$ is contained in that of $C^{k} \cup M^{k} \cup\left(L^{k} \cap S_{k}\right)$. In particular, $|\zeta|$ cannot contain $\left|v_{1}\right|$ as a component since $v_{1}$ intersects $C^{k} \cup M^{k} \cup\left(L^{k} \cap S_{k}\right)$ essentially.

We shall now show that $\zeta$ cannot intersect $v_{1}$ essentially. Suppose, on the contrary, that there is a component $\zeta_{0}$ of $\zeta$ intersecting $v_{1}$ essentially. We shall first consider the case when $\zeta_{0}$ is contained in $\widehat{F}$. Since $\rho_{n}$ is dual to $\zeta_{n}$ whose support is equal to $|\zeta|$ for large $n$, there is a $\pi_{1}\left(S_{k}\right)$-equivariant map $q_{n}: \mathbb{H}^{2} \rightarrow \mathcal{T}_{n}$ which maps each component of the preimage of $\zeta$ to a point in $\mathcal{T}_{n}$. Then there is a train track $\tau$ carrying $\zeta_{0}$ each component of whose preimage is mapped to a point by $q_{n}$, as was shown in the proof of Theorem 4 of Kleineidam and Souto [27]. Let $s_{n} k_{n}$ be a sequence of weighted simple closed curves converging to $\zeta_{0}$ which is carried by $\tau$. (Such a sequence can be taken by approximating the weight system for $\zeta_{0}$ by rational numbers.) Then each lift of $k_{n}$ in $\mathbb{H}^{2}$ is mapped to a point by $q_{n}$; hence the translation length of $\rho\left(k_{n}\right)=\left(\pi_{n}\right)_{*} \rho_{n}\left(k_{n}\right)$ is 0 . On the other hand, since $\zeta_{0}$ intersects $v_{1}$, by homotoping $\zeta_{0}$ and $v_{1}$ in $V_{k}$ into $F$, we see that the translation length of $\rho\left(k_{n}\right)$ must go to $\infty$ if $\zeta_{0}$ is not a simple closed curve, and is positive if $\zeta_{0}$ is a simple closed curve. This is a contradiction.

Next we consider the case when $\zeta_{0}$ is not contained in $\hat{F}$. Since $\zeta$ is a measured lamination, the leaves of $\zeta$ intersecting $\partial \hat{F}$ cannot accumulate inside $\hat{F}$, and consist of finitely many parallel families of geodesic arcs with endpoints on $\partial \widehat{F}$. By joining geodesic arcs in parallel families and arcs on $\partial F$, we can construct essential simple closed curves on $\hat{F}$ with null translation length under $\rho$. Since $\zeta_{0}$ intersects $v_{1}$ essentially, so does one of the simple closed curves, which we denote by $\gamma$. This curve $\gamma$ is homotopic to a simple closed curve $\gamma^{\prime}$ on $F$ which intersects $v_{0}$ essentially. Since the restriction of $\rho$ to $\pi_{1}(F)$ is dual to $v$ whose support contains that of $v_{0}$, we see that $\rho(\gamma)$ has a positive translation length in $\mathcal{T}$. This contradicts the fact that $\rho(\gamma)$ has null translation length in $\mathcal{T}$.

Thus we have shown that $\zeta$ cannot intersect $v_{1}$ essentially. The only remaining possibility is that $v_{1}$ is disjoint from $\zeta$. In this case, we consider a complementary 
region $U$ of $\zeta$ containing $v_{1}$. We note that $U \cap \widehat{F}$ contains $T\left(v_{1}\right)$. If $v_{1}$ is not a weighted simple closed curve, then there is a simple closed curve $\delta$ in $T\left(v_{1}\right) \subset U \cap \widehat{F}$ intersecting $v_{1}$ essentially. Since $\delta$ is disjoint from $\zeta$, the translation length of $\rho_{n}(\delta)$, hence also that of $\rho(\delta)$, is 0 . On the other hand, $\delta$ is homotopic to a simple closed curve $\delta^{\prime}$ on $F$ intersecting $v_{0}$ essentially. Therefore $\rho(\delta)$ must have positive translation length in $\mathcal{T}$ as before. This is a contradiction.

Next suppose that $v_{1}$ is a weighted simple closed curve. By our choice of $v_{0}$, there is a component $\xi$ of $C^{k} \cup M^{k} \cup\left(L^{k} \cap S_{k}\right)$ intersecting $v_{1}$ essentially. Since $\zeta$ is assumed to be disjoint from $v_{1}$, there is a complementary region $U$ of $\zeta$ such that $U \cap \widehat{F}$ contains $v_{1} \cup \xi$. If $U \cap \widehat{F}$ contains a simple closed curve intersecting $v_{1}$ essentially, we are done by arguing as in the previous paragraph. Otherwise, we take a simple geodesic arc $\beta$ in $U \cap \widehat{F}$ with endpoints at $\partial \widehat{F}$ intersecting $v_{1}$ essentially. Starting from $\beta$, and using arcs on $\partial \hat{F}$ and geodesic arcs in parallel families of $\zeta \cap \widehat{F}$ intersecting $\partial \hat{F}$, as in the previous case when $\zeta_{0}$ intersects $v_{1}$ but is not contained in $\widehat{F}$, we can construct a simple closed curve $\gamma$ in $\widehat{F}$ which intersects $v_{1}$ essentially and has null translation length under $\rho$. So we obtain a contradiction in the same way as before. Thus, we have a contradiction in every case, completing the proof.

Thus we have shown that either $V$ or $\mathcal{B}$ must be empty. If $V$ is empty, by Corollary 4.2, we are done. If $\mathcal{B}$ is empty, then by Proposition 5.4, for every component $V_{k}$ of $V$, the restriction $\phi_{i} \mid H^{k}$ converges in $A H\left(H^{k}\right)$. Since $\mathcal{B}$ is empty, the restriction of $\phi_{i}$ to each component $W$ of the boundary-irreducible part also converges (after taking a subsequence and conjugates). Then, the same argument as the proof of Lemmata 4.5 and 4.6 in [50] shows Theorem 3.3.

\section{Unrealisable laminations and ending laminations}

Having proved Theorem 3.3, we now know that $\left\{q\left(v_{i}\right)\right\}$ converges in $A H(G)$ after passing to a subsequence. Let $\phi: G \rightarrow \mathrm{PSL}_{2} \mathbb{C}$ be a representation with image $\Gamma=\phi(G)$ such that $(\Gamma, \phi)$ is the limit of (a subsequence of ) $\left\{q\left(v_{i}\right)\right\}$ in $A H(G)$. We consider the hyperbolic 3-manifold $M_{\Gamma}$. We use the symbol $\Phi$ to denote a homotopy equivalence from $M_{G}$ to $M_{\Gamma}$ induced by the isomorphism $\phi$. Let $C^{\prime}$ be a relative compact core of $\left(M_{\Gamma}\right)_{0}$. If we denote $C^{\prime} \cap \partial\left(M_{\Gamma}\right)_{0}$ by $P^{\prime}$, then the pair $\left(C^{\prime}, P^{\prime}\right)$ is a pared manifold.

In this section, we shall prove that for the given laminations $\mu_{j}$ in Theorem 1.2, their images $\Phi\left(\mu_{j}\right)$ actually represent ending laminations of ends of $\left(M_{\Gamma}\right)_{0}$ (Proposition 6.5). A similar result on the equivalence of being unrealisable and representing an ending 
lamination was given independently by Namazi and Souto [43] as we mentioned in the Introduction (Section 1).

Before stating the main proposition, we shall show that $\Phi$ can be homotoped to take $(C, P \cup T)$ to $\left(C^{\prime}, P^{\prime}\right)$ and that $\Phi\left(\mu_{j}\right)$ is unrealisable in $M_{\Gamma}$, where $P$ and $T$ are as given in Theorem 1.2.

Lemma 6.1 The homotopy equivalence $\Phi$ can be homotoped so that $\Phi(C, P \cup T) \subset$ $\left(C^{\prime}, P^{\prime}\right)$ as pairs and $\Phi \mid(P \cup T)$ is an embedding into $P^{\prime}$.

Proof Since $C^{\prime}$ is a compact core, we can homotope $\Phi$ so that $\Phi(C) \subset C^{\prime}$. Since any immersed incompressible torus in $C^{\prime}$ is homotopic into (a component of ) the union of torus components of $P^{\prime}$, which we denote by $T^{\prime}$, we can make $\Phi(T) \subset T^{\prime}$. Let $T_{0}$ be a component of $T$. Then, since $\pi_{1}\left(T_{0}\right)$ is a maximal abelian subgroup in $\pi_{1}(C)$, we see that $\Phi \mid T_{0}$ induces an isomorphism from $\pi_{1}\left(T_{0}\right)$ to $\pi_{1}\left(T_{0}^{\prime}\right)$ for some component $T_{0}^{\prime}$ of $T^{\prime}$, hence is homotopic to a homeomorphism to $T_{0}^{\prime}$. Thus we have shown that $\Phi$ can be homotoped so that $\Phi \mid T$ is an embedding into $T^{\prime}$.

Let $c$ be a core curve of a component of $P$. By the definition of $v_{i}$, we have length $_{v_{i}}(c) \rightarrow 0$. By Theorem 6.2 in Sugawa [57] or the main theorem of Canary [18], this implies that length $\left(\phi_{i}(c)\right) \rightarrow 0$, hence also that $\phi(c)$ is parabolic. Therefore, $\Phi$ can be homotoped so that $\Phi(c) \subset P^{\prime}$. Since $c$ represents a generator of a maximal abelian group, (for $(C, P \cup T)$ is a pared manifold,) $\Phi \mid c$ is homotopic to a homeomorphism to a core curve of an annulus component of $P^{\prime}$. This completes the proof.

\subsection{Unrealisability of $\mu_{j}$}

Recall that the aim of this section is to show that $\Phi \mid \mu_{j}$ represents an ending lamination for some end of $\left(M_{\Gamma}\right)_{0}$. We shall first see that $\Phi \mid \mu_{j}$ cannot be realised by a pleated surface. Recall that $\mu_{j}$ is contained in a component $\Sigma_{j}$ of $\partial C \backslash(P \cup T)$.

Lemma 6.2 There is no pleated surface homotopic to $\Phi \mid \Sigma_{j}$ realising $\mu_{j}$.

To prove this lemma, we need to invoke the following lemma which appeared as Lemma 4.10 in [49]. Although we allow $\Gamma$ to have parabolic elements here, the proof in [49] works with only a slight refinement as we shall see below.

In the following, we say that a map $f: \Sigma \rightarrow M_{\Gamma}$ is adapted to a tied neighbourhood $N$ of a train track $\tau$ on $\Sigma$ if it maps each branch of $\tau$ to a geodesic segment and each tie of $N$ to a point. 
Lemma 6.3 Let $\Sigma$ be a component of $\partial C \backslash P$. Suppose that $\mu$ is an arational measured lamination in $\mathcal{M}(\Sigma)$ which can be realised by a pleated surface $f: \Sigma \rightarrow M_{\Gamma}$. Let $\left\{w_{k} c_{k}\right\}$ be a sequence of weighted essential simple closed curves which converges to $\mu$. Then for any $\delta>0$ and $t<1$, there exist a continuous map $h: \Sigma \rightarrow M_{\Gamma}$ homotopic to $f$ and a subsequence $\left\{w_{k(l)} c_{k(l)}\right\}$ of $\left\{w_{k} c_{k}\right\}$ with the following two properties.

(i) The map $h$ is adapted to a tied neighbourhood $N$ of a train track $\tau$ which carries $\mu$ and the $w_{k(l)} c_{k(l)}$ for sufficiently large $l$. Moreover, $N$ can be taken to contain $\mu$ and $c_{k(l)}$ in such a way that their leaves are transverse to the ties of $N$ (without moving $\mu$ and $c_{k(l)}$ by a homotopy).

(ii) For sufficiently large $l$, the simple closed curve $h\left(c_{k(l)}\right)$ represents a loxodromic class. Moreover the closed geodesic $c_{k(l)}^{*}$ in $M_{\Gamma}$ homotopic to $h\left(c_{k(l)}\right)$ has a part with length at least $t$ length $h\left(c_{k(l)}\right)$ which is contained in the $\delta$ neighbourhood of the closed curve $h\left(c_{k(l)}\right)$.

Proof We need to show that the argument in [49] works even if we allow parabolic elements to exist. It is easy to see that parabolic elements corresponding to punctures of $\Sigma$ do not affect the argument.

We shall discuss the case when there may be a closed curve not homotopic to a puncture of $\Sigma$ which represents a parabolic class of $\Gamma$. (Such an element is called an accidental parabolic element in some literature.) We need to show that for every $\left\{w_{k} c_{k}\right\}$ converging to $\lambda$, after passing to a subsequence, every $h\left(c_{k}\right)$ represents a loxodromic class of $\Gamma$. Once we prove this, the argument involving the area estimate of piecewise geodesic annulus cobounded by $h\left(c_{k(l)}\right)$ and $c_{k(l)}^{*}$, which is originally due to Bonahon [7], works in the same way as in [49].

Now suppose that this is not the case. Then, by extracting a subsequence, we can assume that all the $h\left(c_{k}\right)$ represent parabolic elements. (It is impossible that infinitely many $h\left(c_{k}\right)$ are null-homotopic since $\mu$ is contained in the Masur domain.) Then we can construct a piecewise geodesic ideal annulus $A_{k}$ cobounded by $h\left(c_{k}\right)$ and a cusp. The area of this annulus is equal to the total exterior angle $e_{k}$ of $h\left(c_{k}\right)$. We can make each of the exterior angles and $w_{k} e_{k}$ arbitrarily small and the length of the image of each branch of $\tau$ arbitrarily long by approximating a pleated surface realising $\lambda$ by $h$ closely. (See Lemma 4.8 of [49].) For each point of $\partial A_{k}=h\left(c_{k}\right)$, we consider a geodesic on $A_{k}$ (with respect to the two-dimensional hyperbolic metric induced on $A_{k}$ ) perpendicular to $\partial A_{k}$. By the Gauss-Bonnet formula, these geodesic arcs are disjoint and can be extended indefinitely if they start outside the $\eta$-neighbourhoods of the vertices, where $\eta$ goes to 0 as the exterior angle goes to 0 . Since $w_{k}$ length $h\left(c_{k}\right)$ converges to a positive constant, the area of $A_{k}$ multiplied by $w_{k}$ goes to infinity 
as $h$ approximates the pleated surface realising $\lambda$ closer and closer. This contradicts the fact shown above that $w_{k} e_{k}$, which is equal to $w_{k} \operatorname{Area}\left(A_{k}\right)$, goes to 0 .

Proof of Lemma 6.2 Suppose $\mu_{j}$ is realised by a pleated surface $g_{\infty}:\left(\Sigma_{j}, n_{\infty}\right) \rightarrow$ $M_{\Gamma}$ homotopic to $\Phi \mid \Sigma_{j}$. Then every measured lamination on $\Sigma_{j}$ with the same support as $\mu_{j}$ is also realised by this pleated surface. Let $\left\{\gamma_{k}\right\}$ be a sequence of simple closed curves on $\Sigma_{j}$ such that $\left\{w_{k} \gamma_{k}\right\}$ converges to a measured lamination with the same support as $\mu_{j}$, and $\gamma_{k}^{*}$ the closed geodesic homotopic to $\Phi\left(\gamma_{k}\right)$. Since $w_{k}$ length $_{n_{\infty}}\left(\gamma_{k}\right)$ converges to length $n_{\infty}\left(\mu_{j}\right)$, and length $\left(\gamma_{k}^{*}\right) \leq$ length $_{n_{\infty}}\left(\gamma_{k}\right)$, we see that length $\left(\gamma_{k}^{*}\right)$ grows at most in the order of $w_{k}^{-1}$ as $k \rightarrow \infty$.

Let $g_{k}:\left(\Sigma_{j}, n_{k}\right) \rightarrow M_{\Gamma}$ be a pleated surface homotopic to $\Phi \mid \Sigma_{j}$ which realises $\gamma_{k}$ as $\gamma_{k}^{*}$. We shall show that we can assume that the $g_{k}$ intersect cusp neighbourhoods of $M_{\Gamma}$ only at a thin neighbourhood of the frontier of $\Sigma_{j}$, ie, that $g_{k}\left(\Sigma_{j}\right)$ is disjoint from a sufficiently small cusp neighbourhood corresponding to accidental parabolic elements for every $k$ if we take a subsequence.

Suppose that this is not the case. Then passing to a subsequence, the image of $g_{k}$ outside a neighbourhood of the frontier goes deeper and deeper into a cusp neighbourhood as $k \rightarrow \infty$, for there are only finitely many cusps in $M_{\Gamma}$. Since a pleated surface can intersect a small cusp-neighbourhood only at its thin annulus (for the diameters of the thick parts of the pleated surfaces are uniformly bounded), there is a nonperipheral simple closed curve $d_{k}$ such that $\phi\left(d_{k}\right)$ either represents an accidental parabolic element or is null-homotopic, and $g_{k}\left(d_{k}\right)$ is contained in the $\epsilon_{k}$-cusp neighbourhood $U_{\epsilon_{k}}$ of the same cusp with $\epsilon_{k} \searrow 0\left(\epsilon_{k}\right.$ goes monotonically to 0$)$ as $k \rightarrow \infty$ after passing to a subsequence. Furthermore, any point $x$ such that $g_{k}(x)$ is contained in $U_{\epsilon_{k}}$ is contained in the $\eta_{k}$-thin part of $\Sigma_{j}$ with respect to $n_{k}$ where $\eta_{k} \rightarrow 0$ as $k \rightarrow \infty$.

Take positive numbers $v_{k}$ so that $\left\{v_{k} d_{k}\right\}$ converges to a nonempty measured lamination $\xi$ after passing to a subsequence. Suppose that $i\left(\xi, \mu_{j}\right)=0$. Since $\mu_{j}$ is arational, this implies that the supports of $\xi$ and $\mu_{j}$ coincide. Since $\xi$, which has the same support as $\mu_{j}$, is realised by a pleated surface homotopic to $\Phi \mid \Sigma_{j}$ realising $\mu_{j}$, by Lemma $6.3, \phi\left(d_{k}\right)$ must represent a loxodromic element. Since $\phi\left(d_{k}\right)$ is either parabolic or trivial, this is a contradiction. Thus we have $i\left(\xi, \mu_{j}\right)>0$. Then $i\left(d_{k}, \gamma_{k}\right)$ grows in the order of $w_{k}^{-1} v_{k}^{-1}$.

Now, let $D_{k}$ be the distance between $\partial U_{\epsilon_{1}}$ and $U_{\epsilon_{k}}$. Since $\epsilon_{k} \searrow 0$, we have $D_{k} \rightarrow \infty$ as $k \rightarrow \infty$ by Brooks and Matelski [13]. For each intersection $p$ of $\gamma_{k}$ with $d_{k}$, there is an arc $a_{p}$ on $\gamma_{k}$ containing $p$ such that $g_{k}\left(a_{p}\right)$ starts from $\partial U_{\epsilon_{1}}$, goes into $U_{\epsilon_{k}}$ and comes back to $\partial U_{\epsilon_{1}}$. Then $g_{k}\left(a_{p}\right)$ has length at least $2 D_{k}$. (See Figure 1.) Since each intersection corresponds to an arc traversing the $\epsilon_{k}$-thin annulus around $d_{k}$, we see that 
$a_{p} \cap a_{p^{\prime}}=\varnothing$ if $p \neq p^{\prime}$. It follows that the geodesic $\gamma_{k}^{*}$ has part with length of order $2 w_{k}^{-1} v_{k}^{-1} D_{k}$. Since $v_{k} d_{k}$ converges in $\mathcal{M L}\left(\Sigma_{j}\right)$, we see that $v_{k}$ is bounded above as $k \rightarrow \infty$. Therefore $v_{k}^{-1} D_{k}$ goes to $\infty$ as $k \rightarrow \infty$, which implies $w_{k}^{-1} v_{k}^{-1} D_{k}$, hence also length $\left(\gamma_{k}^{*}\right)$, grows in a higher order than $w_{k}^{-1}$. This contradicts the fact that length $\left(\gamma_{k}^{*}\right)$ grows at most in the order of $w_{k}^{-1}$, which was proved above.

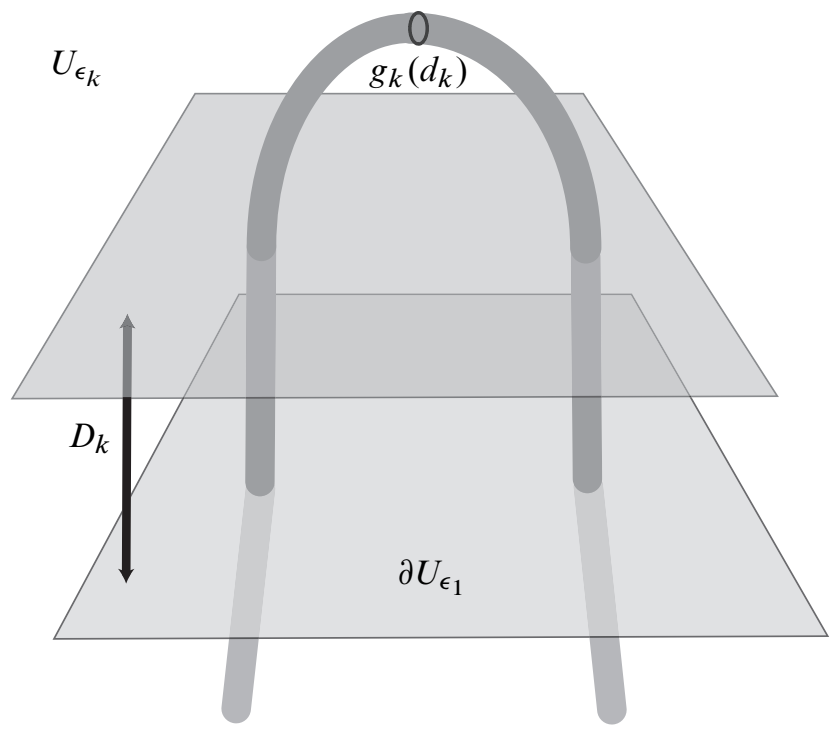

Figure 1: Each intersection with $d_{k}$ contributes $2 D_{k}$ to the length of $\gamma_{k}^{*}$.

Thus, we have shown that $g_{k}\left(\Sigma_{j}\right)$ does not intersect cusps outside a thin neighbourhood of the frontier; hence there is a uniform upper bound for the diameters of the noncuspidal parts of the pleated surfaces $g_{k}$. Moreover, by Lemma 6.3, $g_{k}\left(\Sigma_{j}\right)$ is within a uniformly bounded distance from $g_{\infty}\left(\Sigma_{j}\right)$. Then, passing to a subsequence, $\left\{g_{k}\right\}$ converges to a pleated surface realising $\mu_{j}$ uniformly on every compact set by the compactness of marked pleated surfaces. (The proof of the compactness is similar to the incompressible case proved in Theorem 5.2.18 of Canary, Epstein and Green [19]. It can be found in the proof of Théorème 2.3 in Otal [52] how to generalise this to the compressible case.) In particular, this implies that length $\left(\gamma_{k}^{*}\right)$ grows in the same order as $w_{k}^{-1}$. The closed geodesic $\gamma_{k}^{*}$ can be projected down to the geometric limit of $\left\{\phi_{i}(G)\right\}$ and then can be pulled back by an approximate isometry to a $\left(K_{k}, \delta_{k}\right)$ quasigeodesic in $M_{\phi_{i}(G)}$ which is homotopic to $\phi_{i}\left(\gamma_{k}\right)$ for sufficiently large $i$, with $K_{k} \rightarrow 1$ and $\delta_{k} \rightarrow 0$. Then we can see that the closed geodesic $\gamma_{i}^{+}$representing the conjugacy class of $\phi_{i}\left(\left[\gamma_{i}\right]\right)$ also has length growing in the same order as $w_{i}^{-1}$ as $i \rightarrow \infty$. (See also the argument in the proof of Lemma 6.12 in [49].) On the other hand, 
as we showed in the proof of Proposition 5.4, there is a sequence of weighted simple closed curves $r_{i} c_{i}$ converging to a measured lamination $\mu_{j}^{\prime}$ having the same support as $\mu_{j}$ such that $r_{i}$ length $\left(\phi_{i}\left(\left[c_{i}\right]\right)\right)$ goes to 0 as $i \rightarrow \infty$. Since in the argument above, $w_{i} \gamma_{i}$ could be taken to be any sequence of weighted simple closed curves converging to a measured lamination with the same support as $\mu_{j}$, we can let it be $r_{i} c_{i}$. Then we reach a contradiction since $r_{i}$ length $\left(\phi_{i}\left(\left[c_{i}\right]\right)\right)$ goes to 0 whereas it was proved above that length $\left(\phi_{i}\left(\left[c_{i}\right]\right)\right.$ grows in the order of $r_{i}^{-1}$. Thus, we have shown that $\mu_{j}$ cannot be realised.

As a consequence of Lemma 6.2, the following holds. (See Lemma 4.4 and Proposition 4.14 in [49], the former of which is based on the argument of Otal [53].)

Corollary 6.4 For any sequence of weighted simple closed curves $\left\{w_{k} \gamma_{k}\right\}$ converging to a measured lamination with the same support as $\mu_{j}$, there is a sequence of pleated surfaces $f_{k}$ homotopic to $\Phi \mid \Sigma_{j}$ which realise the $\gamma_{k}$ and tend to an end $\left(M_{\Gamma}\right)_{0}$ as $k \rightarrow \infty$. Moreover, if $\Sigma_{j}$ is not null-homologous in $C$ relative to $P$, then the end to which the $f_{k}$ tend is topologically tame and has a neighbourhood homeomorphic to $\Sigma_{j} \times \mathbb{R}$ such that $f_{k}$ is homotopic within $\Sigma_{j} \times \mathbb{R}$ to a homeomorphism onto $\Sigma_{j} \times\{\mathrm{pt}\}$.

Even in the case when $\Sigma_{j}$ is null-homologous, the result of Brock and Souto [11] implies that $\Gamma$ is topologically tame.

In this latter case, we do not know a priori if pleated surfaces $f_{k}$ realising $w_{k} \gamma_{k}$ give a product structure near the end. This makes the argument for the latter case more complicated.

\subsection{Main proposition}

In the remainder of this section, we shall show that $\Phi\left(\mu_{j}\right)$ indeed represents an ending lamination.

Proposition 6.5 In the setting of Theorem 1.2, we can homotope $\Phi$ so that the following holds. There is a nice compact core $C^{\prime}$ of $\left(M_{\Gamma}\right)_{0}$ with $P^{\prime}=C^{\prime} \cap \partial\left(M_{\Gamma}\right)_{0}$ such that $\Phi \mid \Sigma_{j}$ is a homeomorphism to a component of $\partial C^{\prime} \backslash P^{\prime}$ and $\Phi\left(\mu_{j}\right)$ represents the ending lamination for the end facing $\Phi\left(\Sigma_{j}\right)$ for every $j=n+1, \ldots, m$.

Since the proof of this proposition is rather long, taking up most of the rest of the paper, we shall first summarise our argument before really starting it. 
Summary of Proof We shall first show that we can reduce the general case to a special case when the compact core $C$ is a compression body. (Lemma 6.6.) Suppose that $C$ is a compression body. Then, we have only to consider the cases when $\Sigma_{j}$ represents a nontrivial second homology class relative to $P$, and when $C$ is a handlebody and $\partial C \backslash P$ is connected. In the former case, which is easier to deal with, a generalised version of Bonahon's intersection lemma (Lemma 6.7) implies that $\Phi$ can be homotoped to a homeomorphism for which $\Phi\left(\mu_{j}\right)$ represents the ending lamination. Most of our discussion will be devoted to the latter case.

In the latter case, we shall first show that $\partial C^{\prime} \backslash P^{\prime}$ is also connected. (Lemma 6.11.) As a corollary of this lemma, we can show that the convergence of $\left\{\left(G_{i}, \phi_{i}\right)\right\}$ to $(\Gamma, \phi)$ is strong. Let $\rho_{i}$ be an approximate isometry between $M_{G_{i}}$ and $M_{\Gamma}$ associated to the geometric convergence of $\left\{G_{i}\right\}$ to $\Gamma$. In the final step, we shall consider a pleated surface $g_{i}: \partial C \rightarrow M_{G_{i}}$ homotopic to $\Phi_{i}$ realising $C^{1} \cup \mu_{1}$. (Note that since $\partial C \backslash P$ was assumed to be connected, there is only one $\mu_{j}$.) We shall then show that $g_{i}$ is incompressible in the complement of $\rho_{i}^{-1}\left(C^{\prime}\right)$. (Lemma 6.17.) Using another generalised version of Bonahon's intersection lemma (Lemma 6.10), we can finally show that $\Phi$ can be homotoped so that $\Phi\left(\mu_{1}\right)$ represents the ending lamination.

Now, we start the proof. First we should remark the following.

Lemma 6.6 To prove Proposition 6.5, it is sufficient to deal with the case when $C$ is a compression body.

Proof Let $\mu_{j}$ be one of $\mu_{n+1}, \ldots, \mu_{m}$. Take a nice compact core $\left(C^{\prime}, P^{\prime}\right)$ of $\left(M_{\Gamma}\right)_{0}$, and homotope $\Phi$ so that $\Phi(C)$ is contained in $C^{\prime}$. Consider the boundary component of $C$ on which $\mu_{j}$, hence also $\Sigma_{j}$ lies, and denote it by $S$. Let $G^{S}$ be a subgroup of $G \cong \pi_{1}(C)$ corresponding to $\iota_{\# \pi_{1}}(S)$, where $\iota_{\#}$ denotes the homomorphism between the fundamental groups induced by the inclusion $\iota: S \rightarrow C$, and set $\Gamma^{S}$ to be $\phi\left(G^{S}\right)$. Then $M_{G}$ has a nice compact core $\bar{C}$ to whose boundary $S$ is lifted homeomorphically as $\bar{S}$. The core $\bar{C}$ is homeomorphic to $S \times I$ if $S$ is incompressible, and is a compression body if $S$ is compressible. Let $\bar{\Sigma}_{j}$ and $\bar{\mu}_{j}$ be the lifts of $\Sigma_{j}$ and $\mu_{j}$ to $\bar{S}$. We denote by $\bar{\Phi}$ a homotopy equivalence from $M_{G}{ }^{S}$ to $M_{\Gamma}$ induced by $\phi \mid G^{S}$.

Now, suppose that Proposition 6.5 is valid under the assumption that a compact core of $M_{G}$ is a compression body. We shall then apply this for $M_{G} s$. Using Lemma 6.1, we can assume that $\Phi \mid P$ is an embedding into $P^{\prime}$. Since we also know that Proposition 6.5 is true for the case when every boundary component of $\partial C \backslash P$ is incompressible by the main theorem of [44], this means that there is a relative compact core $\left(\bar{C}^{\prime}, \bar{P}^{\prime}\right)$ of $\left(M_{\Gamma} S\right)_{0}$ such that $\bar{\Phi} \mid \bar{\Sigma}_{j}$ is a homeomorphism to a component of $\partial \bar{C}^{\prime} \backslash \bar{P}^{\prime}$ facing 
an end $\bar{e}_{j}$ for which $\bar{\Phi}\left(\bar{\mu}_{j}\right)$ represents the ending lamination. Now, Canary's covering theorem proved in [17] shows that there is an end $e_{j}$ of $\left(M_{\Gamma}\right)_{0}$ whose neighbourhood is finitely covered by a neighbourhood of $\bar{e}_{j}$. This also implies that there is a component $\Sigma_{j}^{\prime}$ of $\partial C^{\prime} \backslash P^{\prime}$ such that $\Phi \mid \Sigma_{j}$ is homotopic relative to $P$ to a finite-sheeted covering to $\Sigma_{j}^{\prime}$ for every $j=n+1, \ldots, m$.

For $\Sigma_{j}$ with $j=1, \ldots, n$, we can show that $\Phi \mid \Sigma_{j}$ is a homeomorphism to a boundary component by using a result of Abikoff as follows. Recall that in Definition 3.1, the conformal structure $n_{i}$ on $S$ was taken to converge to $m_{j}$ if restricted to $\Sigma_{j}$. Let $\Omega^{S}$ be a component of the domain of discontinuity of $G$ such that $\Omega^{S} / G^{S}$ is the surface at infinity corresponding to $S$, and $G^{\Sigma_{j}}$ a subgroup of $G^{S}$ corresponding to $\iota_{\#} \pi_{1}\left(\Sigma_{j}\right)$. The quasiconformal deformation $\left(G_{i}, \phi_{i}\right)$ of $G$ is realised by a quasiconformal homeomorphism $h_{i}: S_{\infty}^{2} \rightarrow S_{\infty}^{2}$. By Lemma 3 of Abikoff [1], there is an open subset $\Omega$ of $\Omega^{S}$ invariant under $G^{\Sigma_{j}}$ such that $h_{i} \mid \Omega$ converges to an equivariant homeomorphism to a component $\Omega^{\prime}$ of $\Omega_{\Gamma}$ whose stabiliser is $\phi\left(G^{\Sigma_{j}}\right)$. Since every frontier component of $\Sigma_{j}$ is mapped by $\Phi$ to a closed curve representing a parabolic element, the surface $\Omega^{\prime} / \phi\left(G^{\Sigma_{j}}\right)$ is of finite type. Therefore, there is a frontier component of $\left(M_{\Gamma}\right)_{0}$ facing $\Omega^{\prime} / \phi\left(G^{\Sigma_{j}}\right)$. Since $C^{\prime}$ is a nice compact core, there is a component of $\partial C^{\prime} \backslash P^{\prime}$ isotopic to this frontier component, which we denote by $\Sigma_{j}^{\prime}$. Since $\Sigma_{j}^{\prime}$ carries a group corresponding to $\phi\left(G^{\Sigma_{j}}\right)$ in $\pi_{1}\left(M_{\Gamma}\right) \cong \Gamma$, we can homotope $\Phi \mid \Sigma_{j}$ to a homeomorphism to $\Sigma_{j}^{\prime}$.

Therefore, $\Phi$ is homotopic to a map whose restriction to every component of $\partial C \backslash P$ is a finite-sheeted covering. Since $\Phi \mid P$ is an embedding, this implies that $\Phi \mid \partial C$ is a covering into $\partial C^{\prime}$. Since $\Phi \mid C$ is a homotopy equivalence, by Waldhausen's theorem [59], $\Phi \mid C$ must be homotopic to a homeomorphism to $C^{\prime}$, and in particular $\Phi \mid \Sigma_{j}$ is homotopic to a homeomorphism to $\Sigma_{j}^{\prime}$ even for $j=n+1, \ldots, m$. Furthermore, since $\bar{\Phi}\left(\bar{\mu}_{j}\right)$ represents the ending lamination of $\bar{e}_{j}$, its projection $\Phi\left(\mu_{j}\right)$ represents the ending lamination of the end of $\left(M_{\Gamma}\right)_{0}$ facing $\Sigma_{j}^{\prime}$. This shows Proposition 6.5 for the original $G$ and $\Gamma$.

We assume from now on until the end of the proof of Proposition 6.5 that $C$ is a compression body. Recall that we are considering a component $\Sigma_{j}$ of $\partial C \backslash P$ on which $\mu_{j}$ lies. We need to divide the proof into two parts:

(1) the case when $\Sigma_{j}$ represents a nontrivial second homology class of $H_{2}(C, P)$,

(2) the case when $\Sigma_{j}$ is null-homologous relative to $P$.

Since we are assuming that $C$ is a compression body, (2) corresponds exactly to the case when $C$ is a handlebody and $\partial C \backslash P$ is connected. In both of these cases, we 
need to use the following Lemma 6.7, which is a generalisation of Proposition 3.4 in Bonahon [7]. Before stating the lemma, we shall define some condition for a compact core and a compressible surface, which we need to use in the statement of the lemma.

Let $M$ be a topologically tame hyperbolic 3-manifold, and $C^{\prime}$ a nice compact core of $M_{0}$. If $C^{\prime}$ is boundary-reducible, Proposition 5.1 in Canary [16] implies that we can isotope $C^{\prime}$ so that the following holds: There is a double branched covering $p: \widetilde{M} \rightarrow M$ such that $\widetilde{M}$ admits a pinched negatively curved metric with respect to which $p$ is an isometry to its image if restricted to the complement of $p^{-1}\left(\operatorname{Int} C^{\prime}\right)$, and such that $p^{-1}\left(C^{\prime}\right)$ is a boundary-irreducible compact core of $\widetilde{M}$ whose interior contains the tubular neighbourhood of the branching locus where the metric is deformed.

We call such a compact core $C^{\prime}$ adequate with respect to the covering $p$. We also say that an embedded surface $F$ in $M$ is liftable (with respect to the covering $p$ ) when $F$ lies outside Int $C^{\prime}$ for some adequate $C^{\prime}$, hence in particular is lifted isometrically to $\widetilde{M}$.

The following is a generalisation of Bonahon's intersection lemma in [7], adapted to our settings. What it claims is roughly as follows. We shall consider two closed geodesics situated far away from an adequate core, and project them to closed curves to the boundary of the core. Then, under some condition on the closed curves, it will be shown the intersection number of these closed curves is bounded by $e^{-D}$ times the product of the lengths of the closed geodesics, multiplied by some constant independent of the geodesics, where $D$ is a constant bounding from below the distances from the closed geodesics to their projections.

Lemma 6.7 Set $M=M_{\Gamma}$, and consider an adequate core $C^{\prime}$ of $M$. Let $M_{0^{\prime}}$ be the complement of some of the cusp neighbourhoods of $M$. (This may be equal to $M$ or $M_{0}$ or something in between.) Let $F$ be a properly embedded compact surface in $M_{0^{\prime}}$ separating $U \cong F \times \mathbb{R}$ from $M_{0^{\prime}}$. In the case when $F$ is compressible, we further assume that $F$ is liftable. We fix a hyperbolic metric $m_{F}$ on $F$.

Let $\Sigma$ be a component of $\partial C \backslash P$ and $f: \Sigma \rightarrow M$ a map homotopic to $\Phi \mid \Sigma$ taking the components of the frontier of $\Sigma$ to cusps of $M$. Let $\left\{c_{k}\right\}$ be a sequence of simple closed curves contained in the Masur domain of $\Sigma$ whose projective classes converge to the class represented by a measured lamination $L_{c}$ in the Masur domain of $\Sigma$, and $\left\{d_{k}\right\}$ either another such sequence or a sequence of simple closed curves in the Masur domain of $F$, such that projective classes $\left[d_{k}\right]$ converge to the projective class represented by a measured lamination $L_{d}$ in the Masur domain of either $\Sigma$ or $F$. Suppose that the closed geodesics $c_{k}^{*}$ and $d_{k}^{*}$ which are respectively homotopic to either $f\left(c_{k}\right), f\left(d_{k}\right)$ or $f\left(c_{k}\right), d_{k}$, depending on the definition of $d_{k}$, are contained in $U$ for all $k$. In the 
case when the $d_{k}$ lie on $F$, we further assume that a homotopy between $d_{k}$ and $d_{k}^{*}$ can be taken to be contained in $U \cup F$. Then, taking subsequences of $\left\{c_{k}\right\}$ and $\left\{d_{k}\right\}$ and denoting them by the same symbols, there exist sequences of simple closed curves $\left\{C_{k}\right\}$ on $\Sigma$ and $\left\{D_{k}\right\}$ on either $\Sigma$ or $F$ with the following properties.

(i) Let $\left[\lambda_{c}\right],\left[\lambda_{d}\right]$ be projective laminations to which the projective classes $\left\{\left[C_{k}\right]\right\}$ and $\left\{\left[D_{k}\right]\right\}$ converge respectively. Then we have $i\left(\lambda_{c}, L_{c}\right)=i\left(\lambda_{d}, L_{d}\right)=0$.

(ii) The closed geodesics $C_{k}^{*}, D_{k}^{*}$ homotopic in $M$ to $f\left(C_{k}\right), f\left(D_{k}\right)$ or $f\left(C_{k}\right), D_{k}$ lie in $U$.

(iii) In the case when the $d_{k}$ lie on $F$, a homotopy between $D_{k}^{*}$ and $D_{k}$ can be taken to lie in $U \cup F$.

(iv) Let $\bar{C}_{k}$ be a closed curve on $F$ homotopic to $C_{k}^{*}$ in $U \cup F$. We set $\bar{D}_{k}=D_{k}$ if the $d_{k}$ lie on $F$, and define $\bar{D}_{k}$ to be a closed curve on $F$ homotopic to $D_{k}^{*}$ in $U \cup F$ otherwise. Then we have an inequality

$$
i\left(\bar{C}_{k}, \bar{D}_{k}\right) \leq K e^{-D} \text { length }\left(\bar{C}_{k}\right) \text { length }\left(\bar{D}_{k}\right),
$$

where $D$ is $\min \left\{d\left(\bar{C}_{k}, C_{k}^{*}\right), d\left(\bar{D}_{k}, D_{k}^{*}\right)\right\}$, the length denotes the geodesic length with respect to $m_{F}$, and $K$ is independent of $k$. To be more precise, $K$ depends only the pinching constant for the branched covering $\widetilde{M}$ and a positive constant bounding from below the lengths of essential simple closed curves on $F$ with respect to the metric induced from $M$.

(v) If $c_{k}=d_{k}$, we can take $C_{k}, D_{k}$ to be equal.

Proof This lemma was shown by Bonahon in [7] under the assumptions that $F$ is incompressible and that $c_{k}^{*}$ and $d_{k}^{*}$ can intersect $\epsilon$-Margulis tubes only at their core curves for some fixed positive constant $\epsilon$ by setting $C_{k}=c_{k}, D_{k}=d_{k}$. (In this case, the constant $K$ depends only on a constant bounding the lengths of the essential simple closed curves on $F$ from below. See the argument in the proof of Proposition 3.4 in [7].)

To remove the assumption of incompressibility, still under the assumption that $c_{k}^{*}$ and $d_{k}^{*}$ intersect $\epsilon$-Margulis tubes only at their axes, we apply Canary's construction of a branched covering. Since we assumed that $F$ is liftable, there is a branched covering $p: \widetilde{M} \rightarrow M$, and $F$ is lifted isometrically to an incompressible surface $\widetilde{F}$ separating $\widetilde{F} \times \mathbb{R}$ from $\widetilde{M}_{0^{\prime}}$ (the lift of $M_{0^{\prime}}$ to $\widetilde{M}$ ), which is contained in a component of the complement of the boundary-irreducible compact core $p^{-1}\left(C^{\prime}\right)$ of $\widetilde{M}$. Then we can apply Bonahon's argument for the case of incompressible surface. The only difference is that the metric is not hyperbolic in $\widetilde{M}$. (When we realise homotopies as piecewise totally geodesic ones, they may go out of $U$ and intersect the part where 
the curvature is not constant.) Still the argument works since the sectional curvature is pinched between a negative constant and -1 . This affects the constant $K$, but in such a way that it only depends on the pinching constant of $\widetilde{M}$.

Now we shall see how to deal with the case when the closed geodesics $c_{k}^{*}$ and $d_{k}^{*}$ intersect Margulis tubes outside the core curves. What we are going to show is that we can replace $c_{k}$ with $C_{k}$ and $d_{k}$ with $D_{k}$ in such a way that neither $C_{k}^{*}$ nor $D_{k}^{*}$ intersects thin Margulis tubes outside the core curves. The argument is the same for $c_{k}$ and $d_{k}$ except for the case when $d_{k}$ lies on $F$. In the latter case, we need to show additionally that condition (iii) is satisfied. We shall deal with only $c_{k}$ from now on until the last paragraph of the proof, and shall explain how condition (iii) is satisfied in the latter case at the end of the proof.

Since $c_{k}$ is a simple closed curve in the Masur domain, there is a pleated surface $f_{k}$ properly homotopic to $f$ which realises $c_{k}$ as $c_{k}^{*}$. Since we have only to consider the case when $c_{k}^{*}$ is far enough from $\bar{C}_{k}$ and the diameters of the thick parts of pleated surfaces are uniformly bounded, we can assume that the image of $f_{k}$ is entirely contained in $U$.

Suppose that for any small $\epsilon>0$, there is some $f_{k}(S)$ which intersects an $\epsilon$-Margulis tube although $c_{k}^{*}$ is not the core curve of the tube. Then, passing to a subsequence, we can assume that $f_{k}$ intersects an $\epsilon_{k}$-Margulis tube $T_{k}$ with core curve different from $c_{k}^{*}$ such that $\epsilon_{k} \rightarrow 0$. Let $m_{k}$ denote the hyperbolic metric on $\Sigma$ induced by $f_{k}$ from $M$. Since the diameter of the thick part of $\left(\Sigma, m_{k}\right)$ is uniformly bounded, $f_{k}(\Sigma)$ can intersect $T_{k}$ only its thin part. Replacing $\epsilon_{k}$ if necessary, with the condition that $\epsilon_{k} \searrow 0$ preserved, this implies that $\Sigma$ contains an essential simple closed curve $\gamma_{k}$ with length with respect to $m_{k}$ going to 0 such that $f_{k}\left(\gamma_{k}\right)$ is contained in $T_{k}$.

Let $L_{\gamma}$ be a measured lamination to whose projective class $\left\{\left[\gamma_{k}\right]\right\}$ converges after passing to a subsequence. By our definition of $\gamma_{k}$, the closed curve $f_{k}\left(\gamma_{k}\right)$ is either null-homotopic or homotopic to an iteration of the core curve of $T_{k}$. If $i\left(L_{\gamma}, L_{c}\right)=0$, then $L_{\gamma}$ cannot be contained in $\overline{\mathcal{W C}}(\Sigma)$ since $L_{c}$ is contained in the Masur domain. Therefore $f_{k}\left(\gamma_{k}\right)$ is homotopic to an iteration of the core curve of $T_{k}$. By setting $C_{k}$ to be $\gamma_{k}$, this case is reduced to the case when the $c_{k}^{*}$ intersect Margulis tubes only at their core curves. Note also that $C_{k}^{*}$ lies on the core curve of $T_{k}$, hence is homotopic to $f_{k}\left(C_{k}\right)$ in $T_{k}$, which is contained in $U$ for large $k$. Thus we are done in this case.

Suppose next that $i\left(L_{\gamma}, L_{c}\right)>0$. It follows that $c_{k}$ intersects $\gamma_{k}$ essentially for large $k$. Now, we can show the following claim.

Claim 6.8 In this situation, we can find a piecewise geodesic simple closed curve $\delta_{k}$ on $\left(\Sigma, m_{k}\right)$ as follows, after taking a subsequence with respect to $k$. Here, we assume that $c_{k}$ and $\gamma_{k}$ have been isotoped to closed geodesics with respect to $m_{k}$. 
(a) $\delta_{k}$ consists of two arcs, $a_{k}$ on $c_{k}$ and $b_{k}$ on $\gamma_{k}$.

(b) The free homotopy class of $\delta_{k}$ is constant with respect to $k$.

(c) The length of $a_{k}$ goes to infinity whereas that of $b_{k}$ goes to 0 as $k \rightarrow \infty$.

(d) At the two endpoints, $a_{k}$ comes to $b_{k}$ from the opposite sides.

Proof The proof of this claim is similar to the argument which can be found in Affirmation 2.3.4 of Otal [52]. If we fix a hyperbolic metric $m_{0}$ on $\Sigma$, the simple closed curves $c_{k}$ and $\gamma_{k}$, realised as closed geodesics with respect to $m_{0}$, converge in the Hausdorff topology, after passing to a subsequence, to geodesic laminations $\Lambda_{c}$ and $\Lambda_{\gamma}$ which contain the supports of $L_{c}$ and $L_{\gamma}$ respectively. Since we assumed that $i\left(L_{c}, L_{\gamma}\right)>0$, there is a leaf $\lambda_{c}$ of $\Lambda_{c}$ which is not a simple closed curve and intersects a minimal component $\lambda_{\gamma}$ of $\Lambda_{\gamma}$ transversely. Now, take an arc $a$ on a leaf of $\lambda_{c}$ and $b$ on a leaf of $\lambda_{\gamma}$ such that $a$ meets $\lambda_{\gamma}$ only at its endpoints, $\partial a=\partial b$, and at the endpoints, $a$ comes to $b$ from the opposite sides of the leaf containing $b$.

Since $c_{k}$ converges to $\Lambda_{c} \supset \lambda_{c}$ and $\gamma_{k}$ converges to $\Lambda_{\gamma} \supset \lambda_{\gamma}$, there are arcs $a_{k}$ on $c_{k}$ and $b_{k}$ on $\gamma_{k}$ sharing their endpoints, which converge to $a$ and $b$ on $\left(\Sigma, m_{0}\right)$ with respect to the Hausdorff topology. We set $\delta_{k}=a_{k} \cup b_{k}$. Realise $c_{k}$ and $\gamma_{k}$ as closed geodesics with respect to $m_{k}$ and we denote the arcs on them corresponding to $a_{k}$ and $b_{k}$ by the same symbols. We shall show that these $a_{k}, b_{k}$ and $\delta_{k}$ have the required properties.

The properties (a) and (d) follow from our definition of $a_{k}$ and $b_{k}$. Since $a_{k}$ and $b_{k}$ on $\left(\Sigma, m_{0}\right)$ converge to $a$ and $b$ in the Hausdorff topology, if we take a subsequence, we can assume that the homotopy class of $\delta_{k}$ is constant, which shows (b). Since the length of $\gamma_{k}$ with respect to $m_{k}$ goes to 0 , the length of $b_{k}$ goes to 0 , and the arc $a_{k}$ which traverses a thick annulus around $\gamma_{k}$ must have length going to $\infty$ with respect to $m_{k}$. This completes the proof of our claim.

Now we return to the proof of Lemma 6.7. Recall that $a_{k}$ is mapped geodesically into $c_{k}^{*}$ by $f_{k}$. Since the length of $b_{k}$ goes to 0 , we see that $f_{k}\left(\delta_{k}\right)$ must represent a nontrivial free homotopy class and the closed geodesic $\delta_{k}^{*}$ homotopic to $f_{k}\left(\delta_{k}\right)$ stays within a distance going to 0 as $k \rightarrow \infty$ from $f_{k}\left(\delta_{k}\right)$, and its length goes to infinity as $k \rightarrow \infty$. This is a contradiction since $\delta_{k}$ represents a constant free homotopy class. Thus we have shown that $i\left(L_{\gamma}, L_{c}\right)=0$ always holds and this completes the proof except for the case when the $d_{k}$ lie on $F$.

Now finally, we consider the case when the $d_{k}$ lie on $F$. We consider the inclusion $\iota: F \rightarrow M_{0}$ in place of $f$ in the argument above. All the argument works without any modification except for the proof of condition (iii). We shall now show that 
condition (iii) holds. There is a pleated surface $\iota_{k}: F \rightarrow M_{0^{\prime}}$ realising $d_{k}$ which is homotopic to $\iota$. In general, a homotopy between $\iota$ and $\iota_{k}$ may not lie in $U \cup F$. Still, since $F$ is liftable, there is a simple closed curve $D_{k}$ on $F$ homotopic to $d_{k}^{*}$ in $U \cup F$. The closed curves $D_{k}$ and ${\underset{\sim}{d}}_{k}^{*}$ are lifted to a simple closed curve $\tilde{d}_{k}$ on the lift $\tilde{F}$ of $F$ and a closed geodesic $\tilde{d}_{k}^{*}$ homotopic to $\tilde{d}_{k}$. We can see that for large $k$ there is a pleated surface in $\widetilde{M}_{0^{\prime}}$ realising $\tilde{d}_{k}$ as $\tilde{d}_{k}^{*}$ homotopic to the inclusion of $\tilde{F}$. Such a pleated surface is projected to a pleated surface in $U$ which is homotopic to the inclusion of $F$ in $U \cup F$. By redefining $\iota_{k}$ to be a pleated surface obtained as this and apply the argument as above considering $\iota_{k}$ in place of $f_{k}$, we get condition (iii).

\subsection{The case when $\Sigma_{j}$ is homologically nontrivial}

Now we reformulate and prove Proposition 6.5 in case (1) on page 862.

Lemma 6.9 Suppose that either $C$ is not a handlebody or $\partial C \backslash P$ is disconnected. Let $\left(C^{\prime}, P^{\prime}\right)$ be a nice compact core of $\left(M_{\Gamma}\right)_{0}$ which is adequate with respect to a branched covering. Then, $\Phi$ can be homotoped so that $\Phi \mid \Sigma_{j}$ is a homeomorphism to a component of $\partial C^{\prime} \backslash P^{\prime}$ and $\Phi\left(\mu_{j}\right)$ represents the ending lamination of the end facing that component for every $j=n+1, \ldots, m$.

Proof By Corollary 6.4, there is a sequence $\left\{f_{k}\right\}$ of pleated surfaces which realise $w_{k} \gamma_{k}$ converging to $\mu_{j}$ and tend to a topologically tame end $e$ of $\left(M_{\Gamma}\right)_{0}$ with a neighbourhood homeomorphic to $\Sigma_{j} \times \mathbb{R}$. Let $\gamma_{k}^{*}$ be the closed geodesic homotopic to $\Phi\left(\gamma_{k}\right)$ which is contained in the image of $f_{k}$. By properly isotoping $C^{\prime}$, we can assume that this neighbourhood $\Sigma_{j} \times(0, \infty)$ is a component of the complement of $C^{\prime}$ in $\left(M_{\Gamma}\right)_{0}$ with $\Sigma_{j} \times\{0\}$ identified with a component of $\partial C^{\prime} \backslash P$. Let $\pi$ be the projection of $\Sigma_{j} \times[0, \infty)$ to $\Sigma_{j} \times\{0\}$. Since $f_{k}\left(\Sigma_{j}\right)$ is homotopic to $\Sigma_{j} \times\{\mathrm{pt}\}$ within $\Sigma_{j} \times \mathbb{R}$ by Corollary 6.4, we see that $\pi \circ f_{k}$ is homotopic to a homeomorphism, which we shall denote by $h_{k}$. Note moreover that $h_{k}$ can be extended to a map from $C$ to $C^{\prime}$ inducing an isomorphism between the fundamental groups which is conjugate to $\phi$.

Now, since $e$ is topologically tame and geometrically infinite (for $f_{k}$ tends to $e$ ), there is a measured lamination $\lambda$ on $\Sigma_{j} \times\{0\}$ representing the ending lamination for $e$, which is contained in the Masur domain $\mathcal{M}\left(\Sigma_{j} \times\{0\}\right)$. This means that there is a sequence of weighted simple closed curves $\left\{s_{k} d_{k}\right\}$ converging to $\lambda$ in $\mathcal{M}\left(\Sigma_{j} \times\{0\}\right)$ such that there are closed geodesics $d_{k}^{*}$ in $\Sigma_{j} \times(0, \infty)$ tending to $e$ which are homotopic to the $d_{k}$ in $\Sigma_{j} \times[0, \infty)$. On the other hand, as was seen above, $h_{k}\left(\gamma_{k}\right)$ is homotopic to $\gamma_{k}^{*}$ in $\Sigma_{j} \times[0, \infty)$.

Since both $\gamma_{k}^{*}$ and $d_{k}^{*}$ tend to the end of $\left(M_{\Gamma}\right)_{0}$ contained in $\Sigma_{j} \times[0, \infty)$ as $k \rightarrow \infty$, by Lemma 6.7, there are sequences of simple closed curves $C_{k}$ on $\Sigma_{j}$ and $D_{k}$ on $\Sigma_{j} \times\{0\}$ 
such that $C_{k} /$ length $\left(C_{k}\right)$ and $D_{k} /$ length $\left(D_{k}\right)$ converge to measured laminations $L_{C}, L_{D}$ on $\Sigma_{j}$ and $\Sigma_{j} \times\{0\}$ respectively with $i\left(L_{C}, \mu_{j}\right)=0, i\left(L_{D}, \lambda\right)=0$ and

$$
i\left(\frac{D_{k}}{\text { length }\left(D_{k}\right)}, \frac{h_{k}\left(C_{k}\right)}{\text { length }\left(h_{k}\left(C_{k}\right)\right)}\right) \rightarrow 0 .
$$

This implies that $\left\{h_{k}\left(C_{k}\right) /\right.$ length $\left.\left(h_{k}\left(C_{k}\right)\right)\right\}$ converges to a measured lamination with the same support as $\lambda$ since $\lambda$ is arational, hence in particular, is contained in $\mathcal{M}\left(\Sigma_{j} \times\{0\}\right)$ for large $k$. Since $\mu_{j}$ is also arational and contained in the Masur domain, $L_{C}$ has the same support as $\mu_{j}$, hence in particular is also contained in the Masur domain. Therefore $C_{k}$ lies in the Masur domain of $\Sigma_{j}$ for large $k$.

Since both $\left\{\left[C_{k}\right]\right\}$ and $\left\{\left[h_{k}\left(C_{k}\right)\right]\right\}$ converge in the projectivised Masur domains, and the group of homeomorphisms of $\Sigma_{j} \times\{0\}$ which are homotopic to the identity in $C^{\prime}$ acts on the projectivised Masur domain properly discontinuously, this implies that for sufficiently large $k$, the homeomorphism $h_{k}$ does not depend on $k$. We denote $h_{k}$ for such large $k$ by $h$. Since $\lambda$, hence also $L_{D}$, represents the ending lamination for $e$, so does $h\left(L_{C}\right)$, hence also $h\left(\mu_{j}\right)$. Since $\Phi \mid \Sigma_{j}$ is homotopic to $h$ in $\left(M_{\Gamma}\right)_{0}$, this shows that we can homotope $\Phi$ near $\Sigma_{j}$ so that $\Phi \mid \Sigma_{j}$ is a homeomorphism to $\Sigma_{j} \times\{0\}$ and $\Phi\left(\mu_{j}\right)$ represents the ending lamination for the end facing it. Since we can achieve this only changing $\Phi$ near $\Sigma_{j}$, we can repeat the same operation for each $\Sigma_{j}$, one by one for all $j=n+1, \ldots, m$, and complete the proof.

\subsection{Uniform version of Lemma 6.7 for case (2)}

In Lemma 6.7, the hyperbolic 3-manifold in which the closed geodesics lie is fixed. We need to consider a sequence of hyperbolic 3-manifolds and a pair of sequences of closed geodesics contained in them, one pair in each manifold, for the proof of case (2) of the alternatives on page 862. We start with clarifying the setting of our situation where we need to use the lemma.

Suppose that the nice compact core $C$ of $M_{G}$ is a handlebody and $\partial C \backslash P$ is connected. (This is equivalent to the assumption of (2) since we are assuming that $C$ is a compression body.) Let $C^{\prime}$ be a nice compact core of $\left(M_{\Gamma}\right)_{0}$ as before. Since $\Gamma \cong G$ is a free group, $C^{\prime}$ is also a handlebody. We further assume that $\partial C^{\prime} \backslash P^{\prime}$ is connected. (It will be proved in Lemma 6.11 that this always holds in case (2).) Suppose that $\left\{\phi_{i}\right\}$ converges to $\phi$ strongly, ie $\left\{\phi_{i}(G) \in A H(G)\right\}$ converges to $\Gamma=\phi(G)$ also geometrically. (This will be proved to be the case in Lemma 6.14.) Set $M_{i}=M_{\phi_{i}(G)}$ and $M=M_{\Gamma}$. Fix a branched covering $p_{\infty}: \widetilde{M} \rightarrow M$ obtained by Canary's construction as was explained just before Lemma 6.7 . Since $\left\{G_{i}\right\}$ converges strongly to $\Gamma$, we can take branched coverings $p_{i}: \widetilde{M}_{i} \rightarrow M_{i}$ so that $\widetilde{M}_{i}$ converges geometrically to $\widetilde{M}$ and approximate 
isometries are equivariant with respect to the covering translations. (Refer to Chapter 5 of [49] for a proof of this fact.) We assume that $\left(C^{\prime}, P^{\prime}\right)$ is a nice compact core of $M$ which is adequate with respect to $p_{\infty}$. Let $F_{\infty}$ be $\partial C^{\prime} \backslash P^{\prime}$ for this adequate $C^{\prime}$ separating $U \cong F_{\infty} \times \mathbb{R}$ from $M_{0}$. We denote by $\rho_{i}$ an approximate isometry between $M_{i}$ and $M$, which we can assume to be $C^{\infty}$, and set $F_{i}=\rho_{i}^{-1}\left(F_{\infty} \cup P^{\prime}\right)=\rho_{i}^{-1}\left(\partial C^{\prime}\right)$. We fix a hyperbolic metric $m_{\partial C^{\prime}}$ on $F_{\infty}$, and let $m_{F_{i}}$ be its pullback $\rho_{i}^{*}\left(m_{\partial C^{\prime}}\right)$. Suppose that $F_{i}$ is an embedded surface in $\left(M_{i}\right)_{0}$ which is liftable with respect to $p_{i}$ and separates $U_{i} \cong F_{i} \times \mathbb{R}$ from $\left(M_{i}\right)_{0}$. (We shall show that we can take $C^{\prime}$ so that this is the case in Lemma 6.15.)

Let $\Sigma$ be $\partial C \backslash P$, which we assumed to be connected. Also, let $f_{i}: \Sigma \rightarrow M_{i}$ be a map taking each component of the frontier to a closed geodesic such that $\rho_{i} \circ f_{i}$ converges uniformly on every compact set in Int $\Sigma$ to $f: \Sigma \rightarrow M$ homotopic to $\Phi \mid \Sigma$ taking each component of the frontier to a cusp.

Lemma 6.10 In this situation, suppose that $\left\{c_{k}\right\}$ is a sequence of simple closed curves on $\Sigma$ such that $\left\{r_{k} c_{k}\right\}$ converges in the Masur domain of $\Sigma$ to a measured lamination $L_{c}$ and that $\left\{d_{k}\right\}$ is one on either $\Sigma$ or $F_{\infty}$ such that $\left\{s_{k} d_{k}\right\}$ converges in the Masur domain of either $\Sigma$ or $F_{\infty}$ to a measured lamination $L_{d}$. Suppose that the closed geodesics $c_{i}^{*}$ and $d_{i}^{*}$ which are homotopic to $f_{i}\left(c_{i}\right)$ and either $f_{i}\left(d_{i}\right)$ or $\rho_{i}^{-1}\left(d_{i}\right)$ respectively are contained in $U_{i}$ for each $i$, and that both $d_{M_{i}}\left(c_{i}^{*}, F_{i}\right)$ and $d_{M_{i}}\left(d_{i}^{*}, F_{i}\right)$ go to $\infty$ as $i \rightarrow \infty$. Furthermore, we assume that $d_{i}^{*}$ is homotopic to $\rho_{i}^{-1}\left(d_{i}\right)$ in $U_{i} \cup F_{i}$ in the case when the $d_{k}$ lie on $F_{\infty}$. Then, after taking a subsequence of $\left\{\phi_{i}\right\}$, there exist sequences of simple closed curves $\left\{C_{i}\right\}$ on $\Sigma$ and $\left\{D_{i}\right\}$ on either $\Sigma$ or $F_{\infty}$ depending on where the $d_{k}$ lie, with the following properties.

(i) In the projective lamination space of $\Sigma$ and that of either $\Sigma$ or $F_{\infty}$ respectively, $\left\{\left[C_{i}\right]\right\}$ converges to a projective lamination $\left[\mu_{c}\right]$ and $\left\{\left[D_{i}\right]\right\}$ converges to $\left[\mu_{d}\right]$ such that $i\left(\mu_{c}, L_{c}\right)=0$ and $i\left(\mu_{d}, L_{d}\right)=0$.

(ii) The closed geodesics $C_{i}^{*}, D_{i}^{*}$, which are homotopic in $M_{i}$ to $f_{i}\left(C_{i}\right)$ and either $f_{i}\left(D_{i}\right)$ or $\rho_{i}^{-1}\left(D_{i}\right)$ respectively, lie in $U_{i}$.

(iii) The closed geodesic $D_{i}^{*}$ is homotopic to $\rho_{i}^{-1}\left(D_{i}\right)$ in $U_{i} \cup F_{i}$ in the case when the $d_{k}$ lie on $F_{\infty}$.

(iv) Let $\bar{C}_{i}, \bar{D}_{i}$ be the closed curves on $F_{i}$ homotopic to $C_{i}^{*}, D_{i}^{*}$ in $U_{i} \cup F_{i}$. (This implies that $\bar{D}_{i}=D_{i}$ in the case when the $d_{k}$ lie on $F_{\infty}$.) Then $\bar{C}_{i}, \bar{D}_{i}$ are disjoint from $\rho_{i}^{-1}\left(P^{\prime}\right)$, and we have

$$
\frac{i\left(\bar{C}_{i}, \bar{D}_{i}\right)}{\operatorname{length}\left(\bar{C}_{i}\right) \text { length }\left(\bar{D}_{i}\right)} \rightarrow 0,
$$

where length denotes the geodesic length with respect to $m_{F_{i}}$.

(v) If $c_{k}=d_{k}$, then we can take $C_{k}$ and $D_{k}$ to be equal. 
Proof First we consider the case when $c_{i}^{*}, d_{i}^{*}$ do not intersect $\epsilon$-Margulis tubes outside their core curves. The infimum of the lengths of essential closed curves on $F_{i}$ with respect to the metric induced from $M_{i}$ converge to that of $F_{\infty}$ with respect to the metric induced from $M$, hence a lower bound of the lengths can be taken to be independent of $i$. On the other hand, since $\widetilde{M}_{i}$ converges to $\widetilde{M}$ geometrically, and since $\widetilde{M}_{i}$ has a hyperbolic metric outside a compact set which converges to a compact set of $\widetilde{M}$ as $i \rightarrow \infty$, there is a negative constant uniformly bounding the sectional curvatures of the $\widetilde{M}_{i}$ below. Since the constant $K$ in Lemma 6.7 depends only on these two, we see that we get the conclusion by setting $C_{i}=c_{i}, D_{i}=d_{i}$ in this case.

Now we shall consider the case when for any small $\epsilon>0$, there exists $i$ such that the closed geodesic $c_{i}^{*}$ or $d_{i}^{*}$ intersects an $\epsilon$-Margulis tube of $M_{i}$ outside the core curve. As in the proof of Lemma 6.7, we have only to consider $c_{i}^{*}$. By extracting a subsequence with regard to $i$, we can assume that there exists $\epsilon_{i} \rightarrow 0$ such that, the closed geodesic $c_{i}^{*}$, which lies in $M_{i}$, intersects an $\epsilon_{i}$-Margulis tube $T_{i}$ in $M_{i}$ outside the core curve for each $i$.

Let $h_{i}:\left(\Sigma, \sigma_{i}\right) \rightarrow M_{i}$ be a pleated surface with boundary homotopic to $f_{i}$ relative to Fr $\Sigma$ which realises $c_{i}$ as $c_{i}^{*}$. Let $s$ be a component of the frontier of $\Sigma$. Then $h_{i}(s)$ is a closed geodesic whose length goes to 0 as $i \rightarrow \infty$ since $\phi(s)$ represents a parabolic class whereas $\phi_{i}(s)$ does not. Therefore, $h_{i}(s)$ is the core curve of some Margulis tube for large $i$. This means that each component of the frontier of $\Sigma$ is either disjoint from or contained in $h_{i}^{-1}\left(T_{i}\right)$. By a simple computation of area using a polar coordinate, we see that for any $A \in \mathbb{R}$, there exists $i_{0} \in \mathbb{N}$ such that for $i \geq i_{0}$, if $h_{i}^{-1}\left(T_{i}\right)$ contains a component with inessential boundary components, then one of the inessential boundary components bounds a disc on $\Sigma$ whose area with respect to $\sigma_{i}$ is greater than $A$. We let such $A$ be greater than $\operatorname{Area}(\Sigma)$, which does not depend on $i$ by the Gauss-Bonnet formula. Then all the components of $h_{i}^{-1}\left(T_{i}\right)$ are incompressible on $\Sigma$ for $i \geq i_{0}$, and we see in particular that $h_{i}^{-1}\left(T_{i}\right)$ contains a simple closed curve $\gamma_{i}$ which is essential on $\Sigma$. (We regard even peripheral curves as essential here.)

Passing to a subsequence, we can assume that $\left[\gamma_{i}\right]$ converges to a projective lamination $\left[L_{\gamma}\right]$ in $\mathbb{P} \mathcal{M L}(\Sigma)$. If $i\left(L_{c}, L_{\gamma}\right)=0$, as in Lemma 6.7, $h_{i}\left(\gamma_{i}\right)$ is essential in $M_{i}$ for large $i$. By defining $C_{i}$ to be $\gamma_{i}$, we can apply the argument for the case when the closed geodesics do not intersect Margulis tubes outside the core curves and get the inequality as we wanted. On the other hand, for any core curve $\delta$ of a component of $P^{\prime}$, the geodesic length of $\rho_{i}^{-1}(\delta)$ in $M_{i}$ goes to 0 . Therefore by the same inequality, we see that the projection of $f_{i}\left(C_{i}\right)$ to $F_{i}$ in $F_{i} \cup U_{i}$ is disjoint from $\rho_{i}^{-1}\left(P^{\prime}\right)$ for large $i$.

Next assume that $i\left(L_{c}, L_{\gamma}\right)>0$. Then applying Claim 6.8 in the proof of Lemma 6.7, we get a piecewise geodesic simple closed curve $\delta_{i}$ on $\left(\Sigma, \sigma_{i}\right)$ representing a constant 
free homotopy class with regard to $i$ such that the closed geodesic $\delta_{i}^{*}$ homotopic to $h_{i}\left(\delta_{i}\right)$ lies within a distance going to 0 from $h_{i}\left(\delta_{i}\right)$ and has length going to infinity. This contradicts the fact that $G_{i}$ converges algebraically.

In the case when $d_{k}$ lies on $F_{\infty}$, we define $D_{i}$ to be the projection of $D_{i}^{*}$ to $F_{i}$ in $U_{i} \cup F_{i}$. Then $D_{i}$ converges to a projective lamination $\left[\mu_{d}\right]$ with $i\left(L_{d}, \mu_{d}\right)=0$ since $d_{i}$ is homotopic to $d_{i}^{*}$ in $U_{i} \cup F_{i}$, and we can use the same argument as in the proof of Lemma 6.7 taking into account the fact that $\widetilde{M}_{i}$ converges to $\widetilde{M}$ geometrically.

\subsection{Connectedness of $\partial C^{\prime} \backslash P^{\prime}$ and strong convergence}

Now we return to the general situation of case (2) when $C$ is a handlebody and $\partial C \backslash P$ is connected. Since in this case, $n=0$ and $m=1$ in the statement of Theorem 1.2, we denote the only one $\mu_{j}$, which is $\mu_{1}$, by $\mu$, and $\Sigma_{j}$ by $\Sigma$. Also since $\Gamma$ is free and topologically tame, $M_{\Gamma}$ is homeomorphic to an open handlebody and $\Phi$ is homotopic to a homeomorphism. Therefore, we can assume that $\Phi$ is a homeomorphism.

Lemma 6.11 Let $\left(C^{\prime}, P^{\prime}\right)$ be a nice compact core of $\left(M_{\Gamma}\right)_{0}$ which is adequate with respect to a branched covering. Then, $\partial C^{\prime} \backslash P^{\prime}$ is also connected.

Proof Recall, by Lemma 6.2, we have a sequence of pleated surfaces $f_{k}: \Sigma \rightarrow\left(M_{\Gamma}\right)_{0}$ homotopic to $\Phi \mid \Sigma$ relative to $P$ tending to an end $e$ of $\left(M_{\Gamma}\right)_{0}$, which realise $\left\{w_{k} \gamma_{k}\right\}$ converging to $\mu$ as $k \rightarrow \infty$. Let $\Sigma^{\prime}$ be a component of $\partial C^{\prime} \backslash P^{\prime}$ facing this end $e$. Since $C^{\prime}$ is nice, the component of the complement of $C^{\prime}$ in $\left(M_{\Gamma}\right)_{0}$ facing $\Sigma^{\prime}$ is homeomorphic to $\Sigma^{\prime} \times(0, \infty)$ with $\Sigma^{\prime} \times\{0\}$ identified with $\Sigma^{\prime}$, and the image $f_{k}(\Sigma)$ is contained in $\Sigma^{\prime} \times(0, \infty)$ for every $k$ after passing to a subsequence. This implies that $f_{k}(\Sigma)$ is homotoped within $\Sigma^{\prime} \times(0, \infty)$ into $\Sigma^{\prime} \times\{\mathrm{pt}\}$. By a standard argument as in Section 4.E of [49], in which a subsequence of $\left\{f_{k}\right\}$ is extended to a family of pleated surfaces (allowed to be not constantly curved but negatively curved) realising an half-open arc tending to $\mu$, we can show that the $f_{k}$ are homotopic in $\Sigma^{\prime} \times(0, \infty)$ after passing to a subsequence. Since $\left(f_{k}\right)_{\# \pi_{1}}(\Sigma)$ carries the entire $\pi_{1}\left(C^{\prime}\right) \cong \Gamma$, this implies that $\pi_{1}\left(\Sigma^{\prime}\right)$ also carries the entire $\pi_{1}\left(C^{\prime}\right)$. This means that $\left(C^{\prime}, P^{\prime \prime}\right)$ is either a relative compression body having $\Sigma^{\prime}$ as its exterior boundary or a product $I$-bundle as a pair, where $P^{\prime \prime}$ is the union of the components of $P^{\prime}$ intersecting $\operatorname{Fr} \Sigma^{\prime}$.

If $\left(C^{\prime}, P^{\prime \prime}\right)$ is a relative compression body with empty interior boundary, then $\Sigma^{\prime}=$ $\partial C^{\prime} \backslash P^{\prime}$ and we are done. Therefore, we can assume that $\left(C^{\prime}, P^{\prime \prime}\right)$ is either a relative compression body with nonempty interior boundary or homeomorphic to $\Sigma^{\prime} \times[-1,0]$. In either case, $\partial C^{\prime} \backslash P^{\prime}$ has a component with negative Euler characteristic other than $\Sigma^{\prime}$. On the other hand, we can show the following lemma which contradicts 
this fact. Once we prove this lemma, we shall reach a contradiction and the proof of Lemma 6.11 will be completed.

Lemma 6.12 If $\partial C^{\prime} \backslash P^{\prime}$ is not connected, then $\partial C^{\prime} \backslash P^{\prime}$ cannot have a component with negative Euler characteristic other than $\Sigma^{\prime}$.

Proof of Lemma 6.12 Let $\pi: \Sigma^{\prime} \times[0, \infty) \rightarrow \Sigma^{\prime}$ be the projection to the first factor. Since the $f_{k}$ are all homotopic in $\Sigma^{\prime} \times(0, \infty)$, their projections $\pi \circ f_{k}$ are all homotopic. Note that $f_{k}$ can be extended to a map from $\partial C$ to $\partial C^{\prime}$ by taking each component of $P$ to an annulus on $\operatorname{Fr}\left(M_{\Gamma}\right)_{0}$. Therefore, we can take a map $p: \partial C \rightarrow \Sigma^{\prime}$ with $p(P) \subset P^{\prime \prime}$ whose restriction to $\Sigma$ is homotopic to all the $\pi \circ f_{k}$ relative to $P^{\prime}$. Furthermore this map can be extended to a map $\bar{p}$ from $C$ to $C^{\prime}$ since both of them are handlebodies and $\Phi$ is a homotopy equivalence.

Suppose first that $p \mid \Sigma$ is not a degree-0 map to $\Sigma^{\prime}$ relative to $P^{\prime \prime}$. Then $f_{k}(\Sigma)$ represents a nontrivial second homology class in $\Sigma^{\prime} \times \mathbb{R}$ relative to $\partial\left(M_{\Gamma}\right)_{0}$. Recall that $\left\{f_{k}\right\}$ extends to a continuous family of (negatively curved) pleated surfaces $\rho: \Sigma \times[0, \infty) \rightarrow \Sigma^{\prime} \times[0, \infty)$ such that $\rho(, k)=f_{k}$ and $\rho \mid \Sigma \times\{t\}$ tends to the end $e$ as $t \rightarrow \infty$. Then the argument in Claim 3 of Section 4.E in [49] shows that $\rho$ can be deformed so that $\rho \mid \Sigma \times\left(t_{0}, \infty\right)$ is a homeomorphism onto a neighbourhood of $e$, and in particular that $f_{k}$ is homotopic to a homeomorphism to $\Sigma^{\prime} \times\{t\}$ in $\Sigma^{\prime} \times(0, \infty)$ for large $k$. By extending the homeomorphism to $P$, we see that $\Sigma^{\prime} \cup P^{\prime \prime}$ must be a closed surface homeomorphic to $\partial C$ then. This implies in particular that $\Sigma^{\prime}$ is the only component of $\partial C^{\prime} \backslash P^{\prime}$ contradicting our assumption, and we are done.

Therefore, we can assume that $p \mid \Sigma$ has degree 0 relative to $P^{\prime \prime}$ from now on. Since $p(P) \subset P^{\prime \prime}$, the map $p$ itself is also a degree-0 map from $\partial C$ to $\Sigma \cup P^{\prime \prime}$. Then the simple loop conjecture proved by Gabai [23] gives us an essential simple closed curve $c$ on $\partial C$ such that $p(c)$ inessential on $\Sigma^{\prime} \cup P^{\prime \prime}$. Since $\left(C^{\prime}, P^{\prime \prime}\right)$ is either a relative compression body with exterior boundary $\Sigma^{\prime}$ or a trivial $I$-bundle over $\Sigma^{\prime}$, the union of annuli $P^{\prime \prime}$ is just a collar neighbourhood of $\operatorname{Fr} \Sigma^{\prime}$. Therefore the degree-0 map $p$ from $\partial C$ to $\Sigma^{\prime} \cup P^{\prime \prime}$ can be regarded as a map to $\Sigma^{\prime}$. Since $\Phi$ is a homotopy equivalence, in this situation, $c$ must bound a compressing disc in $C$. Performing compression of $\partial C$ along this disc, we get a surface $\Sigma_{1}$ which contains the complement of a thin neighbourhood of $c$ in $\Sigma$ and is disconnected if the compressing disc is separating, and a map $p^{\prime}: \Sigma_{1} \rightarrow \Sigma^{\prime}$ whose restriction to $\Sigma \cap \Sigma_{1}$ coincides with $p \mid \Sigma \cap \Sigma_{1}$. Let $P_{1}$ be the union of annulus components among $\Sigma_{1} \cap P$. Suppose that there is a component $\bar{\Sigma}_{1}$ of $\Sigma_{1}$ such that $p^{\prime} \mid \bar{\Sigma}_{1}$ has nonzero degree as a map from $\left(\bar{\Sigma}_{1}, P_{1} \cap \bar{\Sigma}_{1}\right)$ to $\left(\Sigma^{\prime}, P^{\prime \prime}\right)$. Then we can also compress $\rho$ to get a map $\rho_{1}: \bar{\Sigma}_{1} \times[0, \infty) \rightarrow \Sigma^{\prime} \times[0, \infty)$ such that $\rho(, t)$ tends to $e$ as $t \rightarrow \infty$. Repeating the previous argument for the nonzero degree 
case, we see that $\rho_{1}\left(\left(\bar{\Sigma}_{1} \backslash\right.\right.$ Int $\left.\left.P_{1}\right) \times\left(t_{0}, \infty\right)\right)$ is a homeomorphism to a neighbourhood of $e$. This implies that $p_{\#}^{\prime}\left(\pi_{1}\left(\bar{\Sigma}_{1}\right)\right)$ carries the fundamental group of $\Sigma^{\prime}$ hence the entire $\pi_{1}\left(C^{\prime}\right)$. This is a contradiction since $\bar{\Sigma}_{1}$ cannot carry the entire fundamental group of $C$. Thus we have shown that $p^{\prime}$ has degree 0 on each component of $\Sigma_{1}$.

Therefore, using this argument repeatedly and extending the map to compressing discs, we can extend $p$ to a map from $C$ to $\Sigma^{\prime}$. Let $\iota$ be the inclusion of $\partial C$ to $C$. What has been shown above implies that $\operatorname{Ker} \iota_{\#} \subset \operatorname{Ker} p_{\#}$. Since $\Phi_{\#}$ is an isomorphism, we see that $\operatorname{Ker} p_{\#} \subset \operatorname{Ker} \iota_{\#}$, hence $\operatorname{Ker} \iota_{\#}=\operatorname{Ker} p_{\#}$.

Now fix a hyperbolic metric on $\Sigma^{\prime}$ which makes each component of the frontier an open end. We need to use the following fact.

Claim 6.13 We can define a continuous map $p_{*}$ from $\mathcal{M}\left(\Sigma^{\prime}\right)$ to the space of the geodesic currents on $\Sigma^{\prime}$ such that

(i) when $\mu$ is a simple closed geodesic in $\mathcal{M}\left(\Sigma^{\prime}\right)$, its image $p_{*}(\mu)$ is the geodesic current corresponding to the closed geodesic homotopic to $p(\mu)$,

(ii) when $p(\mu)$ is homotopic to a measured lamination on $\Sigma^{\prime}$, which we denote again by $p(\mu)$, the geodesic current $p_{*}(\mu)$ coincides with $p(\mu)$ regarded as a geodesic current.

Proof Consider the universal covering $\rho: \mathbb{H}^{2} \rightarrow \Sigma^{\prime}$ and let $\Lambda$ be the limit set in $S_{\infty}^{1}$ of the covering translations by $\pi_{1}\left(\Sigma^{\prime}\right)$. Recall that a geodesic current on $\Sigma^{\prime}$ is defined to be a measure on $\mathcal{G}=(\Lambda \times \Lambda \backslash \Delta) / \mathbb{Z}_{2}$ which is invariant under $\pi_{1}\left(\Sigma^{\prime}\right)$, where $\Delta$ denotes the diagonal and $\mathbb{Z}_{2}$ acts as the interchange of the two factors. (Refer to Bonahon [7; 8].)

Let $\mu$ be a measured lamination contained in the Masur domain of $\Sigma$. By Lemma 5.2, $\mu \cup C^{1}$ lies in $\mathcal{D}(C)$. Let $\widetilde{\partial C}$ be the covering of $\partial C$ associated to the kernel of the homomorphism $\iota_{\#}: \pi_{1}(\partial C) \rightarrow \pi_{1}(C)$ induced from the inclusion $\iota$. Note that the map $p$ is covered by a map between the coverings $\tilde{p}: \widetilde{\partial C} \rightarrow \mathbb{H}^{2}$ since $\operatorname{Ker} \iota_{\#}$ is contained in $\operatorname{Ker} p_{\#}$.

Moreover, since $\operatorname{Ker} \iota_{\#}=\operatorname{Ker} p_{\#}$, the map $\tilde{p}$ is proper and takes the ends of $\widetilde{\partial C}$ to $\Lambda$ injectively. Since $\mu \cup C^{1}$ lies in $\mathcal{D}(C)$, by Lemmata 3.1 and 3.3 in Lecuire [29], each lift of a leaf of $\mu$ connects two distinct points of the ends. It follows that the map $\tilde{p}$ takes a lift of each leaf of $\mu$ to an open arc in $\mathbb{H}^{2}$ ending at two distinct points of $\Lambda$. The ends of $\widetilde{\partial C}$ can be regarded as embedded on the Riemann sphere as the limit set $\Lambda_{0}$ of a Schottky group. (See page 11 of Otal [52] and Section 4 of Kleineidam and Souto [27].) Then the observation above shows that $\tilde{p}$ induces a continuous embedding 
from $\Lambda_{0}$, identified with the set of the ends of $\widetilde{\partial C}$, to $\Lambda$. The transverse measure of $\mu$ defines a measure on $\left(\Lambda_{0} \times \Lambda_{0} \backslash \Delta_{0}\right) / \mathbb{Z}_{2}$ invariant under the covering translation group, where $\Delta_{0}$ denotes the diagonal of $\Lambda_{0} \times \Lambda_{0}$. We push forward this measure to $(\Lambda \times \Lambda \backslash \Delta) / \mathbb{Z}_{2}$ and takes the sum of all of its distinct translates by $\pi_{1}\left(\Sigma^{\prime}\right)$. This geodesic current is defined to be $p_{*}(\mu)$. We can easily check this coincides with the usual definition of geodesic currents corresponding to closed curves when $\mu$ is a simple closed curve in $\mathcal{M}(\Sigma)$. The continuity of $p_{*}$ then follows from the fact that $\Lambda_{0}$ is embedded into $\Lambda$. It is obvious from our construction that in the case when $p(\mu)$ is a measured lamination on $\Sigma^{\prime}$, if we regard $p(\mu)$ as a geodesic current, then it coincides with our geodesic current $p_{*}(\mu)$.

Now, we return to the proof of Lemma 6.12. Let $\lambda$ be a measured lamination representing the ending lamination of the end $e$ facing $\Sigma^{\prime}$. Let $\left\{s_{k} d_{k}\right\}$ be a sequence of weighted simple closed curves converging to $\lambda$ such that the closed geodesic $d_{k}^{*}$ homotopic to $d_{k}$ in $\Sigma^{\prime} \times[0, \infty)$ tends to the end $e$ as $k \rightarrow \infty$.

Regard $w_{k} p\left(\gamma_{k}\right)$ as a weighted closed geodesic with respect to the hyperbolic metric as we fixed before Claim 6.13. Then $w_{k} p\left(\gamma_{k}\right)$ converges to some geodesic current on $\Sigma^{\prime}$ by Claim 6.13. Since the closed geodesic $f_{k}\left(\gamma_{k}\right)$, which is homotopic to $p\left(\gamma_{k}\right)$ in $\Sigma^{\prime} \times[0, \infty)$, tends to the end $e$ in $\Sigma^{\prime} \times[0, \infty)$, by Lemma 6.7, there are sequences of simple closed curves $\left\{C_{k}\right\}$ on $\Sigma$ and $\left\{D_{k}\right\}$ on $\Sigma^{\prime}$ with conditions in the statement of Lemma 6.7 such that

$$
\begin{aligned}
& \lim _{k \rightarrow \infty} i\left(\frac{p_{*}\left(C_{k}\right)}{\text { length }\left(p_{*}\left(C_{k}\right)\right)}, \frac{p_{*}\left(C_{k}\right)}{\text { length }\left(p_{*}\left(C_{k}\right)\right)}\right)=0, \\
& \lim _{k \rightarrow \infty} i\left(\frac{p_{*}\left(C_{k}\right)}{\text { length }\left(p_{*}\left(C_{k}\right)\right)}, \frac{D_{k}}{\text { length }\left(D_{k}\right)}\right)=0 .
\end{aligned}
$$

As in Lemma 6.7, $C_{k} /$ length $\left(C_{k}\right)$ converges to a measured lamination $\mu^{\prime}$ having null intersection number with $\mu$. Since $\mu$ is arational, this means that $\mu^{\prime}$ has the same support as $\mu$. Since $\mu^{\prime}$ is also contained in the Masur domain, by Claim 6.13, $\left\{p_{*}\left(C_{k}\right) /\right.$ length $\left.\left(C_{k}\right)\right\}$ converges to the geodesic current $p_{*}\left(\mu^{\prime}\right)$. We should also note that there is a constant $L$ such that length $\left(p_{*}\left(C_{k}\right)\right) \leq \operatorname{length}\left(p\left(C_{k}\right)\right) \leq L$ length $\left(C_{k}\right)$, where $C_{k}$ is assumed to be a closed geodesic. Note that in contrast, $p\left(C_{k}\right)$ may not be a closed geodesic and length $\left(p\left(C_{k}\right)\right)$ is not the geodesic length. Therefore we have

$$
\begin{aligned}
i\left(p_{*}\left(\mu^{\prime}\right), p_{*}\left(\mu^{\prime}\right)\right) & =\lim _{k \rightarrow \infty} i\left(\frac{p_{*}\left(C_{k}\right)}{\text { length }\left(C_{k}\right)}, \frac{p_{*}\left(C_{k}\right)}{\text { length }\left(C_{k}\right)}\right) \\
& \leq L^{2} \lim _{k \rightarrow \infty} i\left(\frac{p_{*}\left(C_{k}\right)}{\operatorname{length}\left(p_{*}\left(C_{k}\right)\right)}, \frac{p_{*}\left(C_{k}\right)}{\operatorname{length}\left(p_{*}\left(C_{k}\right)\right)}\right)=0 .
\end{aligned}
$$


This means that $p_{*}\left(\mu^{\prime}\right)$ is a measured lamination by Proposition 17 in Bonahon [8]. Since $\mu$ has the same support as $\mu^{\prime}$, this implies that $p_{*}(\mu)$ is also a measured lamination with the same support as $p_{*}\left(\mu^{\prime}\right)$ by our definition of $p_{*}$. On the other hand, $\left\{D_{k} /\right.$ length $\left.\left(D_{k}\right)\right\}$ converges to a measured lamination $\lambda^{\prime}$ such that $i\left(\lambda, \lambda^{\prime}\right)=0$. Since $\lambda$ is arational, this means that $\lambda^{\prime}$ has the same support as $\lambda$. The equation above implies that $i\left(p_{*}\left(\mu^{\prime}\right), \lambda^{\prime}\right)=0$, hence $i\left(p_{*}(\mu), \lambda\right)=0$. Since $\lambda$ is arational, it follows that $p_{*}(\mu)$ has the same support as $\lambda$.

Recall that we denote the union of core curves of $P$ by $C^{1}$. Let $h: C \rightarrow C^{\prime}$ be a homeomorphism inducing $\phi$ between $\pi_{1}(C) \cong G$ and $\pi_{1}\left(C^{\prime}\right) \cong \Gamma$, which we know to exist because both $C$ and $C^{\prime}$ are handlebodies. Recall that $p$ can be extended to a map from $C$ to $C^{\prime}$ which is homotopic to $h$ in $C^{\prime}$ as was shown before Claim 6.13. By Lemma 5.2, we see that $h\left(\mu \cup C^{1}\right)$ is contained in $\mathcal{D}\left(C^{\prime}\right)$.

Consider a convex cocompact hyperbolic metric on $\operatorname{Int} C^{\prime}$, and denote the convex cocompact 3-manifold by $N$. Since $h\left(\mu \cup C^{1}\right)$ lies in $\mathcal{D}\left(C^{\prime}\right)$, it is realised by a pleated surface $g: \partial C^{\prime} \rightarrow N$ homotopic to (a perturbation into Int $C^{\prime}$ of) the inclusion such that $g_{*}$ embeds the lift of $h(\mu)$ into the unit tangent bundle $T_{1}(N)$. (See Theorem 5.5 of Lecuire [28].) Since $p$ extends to a map homotopic to $h$, we see that $p_{*}(\mu)$, which has turned out to be a measured lamination, is homotopic to $h(\mu)$ in $C^{\prime}$. Therefore the support of $h(\mu)$ is homotopic to that of $\lambda$ in $C^{\prime}$, and if we forget the transverse measures, the image of $g \circ h(\mu)$ coincides with the realisation of $\lambda$ by a pleated surface $g^{\prime}: \partial C^{\prime} \rightarrow N$ homotopic to the inclusion.

We see that leaves of $h(\mu)$ isolated from one side are identified with those of $\lambda$ since both $g$ and $g^{\prime}$ induce embeddings into the unit tangent bundle $T_{1}(N)$ of $h(\mu)$ and $\lambda$. Therefore the complementary regions of $\mu$ correspond one-to-one to those of $\lambda$ with the number of sides preserved. Recall that every complementary region of $h(\mu)$ except for the ones containing components of $h\left(C^{1}\right)$ is simply connected. Therefore we see that $\lambda$ cannot a complementary region which has negative Euler characteristic by simply calculating the area.

By the fact which we showed just before Lemma 6.12, we are lead to a contradiction whether $p \mid \Sigma$ has degree 0 or not, if $\left(C^{\prime}, P^{\prime \prime}\right)$ is a relative compression body with nonempty interior boundary or $\left(C^{\prime}, P^{\prime \prime}\right) \cong\left(\Sigma^{\prime}, \partial \Sigma^{\prime}\right) \times I$. Therefore, we see that the only possibility is that $\partial C^{\prime} \backslash P^{\prime}=\Sigma^{\prime}$, which is connected. We have thus completed the proof of Lemma 6.11

The following derives from the covering theorem of Canary [17].

Lemma 6.14 In the present case (2) of the alternatives on page 862, the convergence of $\left\{\left(G_{i}, \phi_{i}\right)\right\}$ to $(\Gamma, \phi)$ is strong. 
Proof By taking a subsequence, we can assume that $\left\{\phi_{i}(G)\right\}$ converges geometrically to a Kleinian group $G_{\infty}$ containing $\Gamma$. Let $q: M_{\Gamma} \rightarrow M_{G_{\infty}}$ be the covering associated to the inclusion $\Gamma \subset G_{\infty}$. Since $\left(M_{\Gamma}\right)_{0}$ has only one end by Lemma 6.11 , which is topologically tame, $q$ must be finite-sheeted by Canary's covering theorem [17]. This implies that $\Gamma=G_{\infty}$ by the argument in Section 9.3 in Thurston [58] (see Lemma 2.3 in Ohshika [46] for a detailed proof).

\subsection{Proof of Proposition 6.5 for the case when $\Sigma$ is null-homologous}

Now we are ready to start the proof of Proposition 6.5 in case (2) on page 862.

Proof of Proposition 6.5 in case (2) Consider a nice compact core $\left(C^{\prime}, P^{\prime}\right)$ of $\left(M_{\Gamma}\right)_{0}$ which is adequate with respect to a branched covering $\widetilde{M} \rightarrow M_{\Gamma}$ by a negatively curved manifold obtained by Canary's construction. Then $\partial C^{\prime} \backslash P^{\prime}$ is liftable, and by Lemma 6.11, is connected. We denote the complement of $C^{\prime}$, which has a product structure, by $\partial C^{\prime} \times(0, \infty)$ identifying $\partial C^{\prime} \times\{0\}$ with $\partial C^{\prime}$.

Set $M_{i}=M_{\phi_{i}(G)}$. Let $x$ be a point in $\mathbb{H}^{3}$, and set its images in $M_{i}$ and $M_{\Gamma}$ under the universal covering projections to be basepoints $x_{i}$ and $x_{\infty}$. Since $\left\{\left(G_{i}, \phi_{i}\right)\right\}$ converges to $(\Gamma, \phi)$ strongly by Lemma 6.14 , there is a $\left(K_{i}, R_{i}\right)$-approximate isometry $\rho_{i}: B_{R_{i}}\left(M_{i}, x_{i}\right) \rightarrow B_{K_{i} R_{i}}\left(M_{\Gamma}, x_{\infty}\right)$ with $R_{i} \rightarrow \infty$ and $K_{i} \rightarrow 1$, which can be assumed to be a diffeomorphism to its image. Furthermore, $\rho_{i}^{-1}\left(C^{\prime}\right)$ is a compact core of $M_{i}$ and $\Phi_{i}$ can be homotoped so that $\Phi_{i} \mid C$ is a homeomorphism onto $\rho_{i}^{-1}\left(C^{\prime}\right)$ for every $i$ if we extract a subsequence. Also, $\rho_{i} \circ \Phi_{i} \mid C$ is homotopic to $\Phi \mid C$ in $M_{\Gamma}$. Take a generator system $g_{1}, \ldots g_{s}$ of $G$, and for each $i$, let $b_{i}$ be a bouquet in $M_{i}$ consisting of the geodesic loops representing $\phi_{i}\left(g_{1}\right), \ldots, \phi_{i}\left(g_{s}\right)$ based at $x_{i}$. We note that the boundary of the convex core of $M_{i}$ is incompressible outside $b_{i}$. The sequence of bouquets $\left\{b_{i}\right\}$ converges geometrically to a bouquet $b_{\infty}$ in $M_{\Gamma}$ based at $x_{\infty}$ representing $\phi\left(g_{1}\right), \ldots, \phi\left(g_{s}\right)$. We can choose an adequate compact core $\left(C^{\prime}, P^{\prime}\right)$ which contains the bouquet $b_{\infty}$. Then $\partial C^{\prime}$ is incompressible outside $b_{\infty}$. Also, passing to a subsequence and enlarging $C^{\prime}$ in its regular neighbourhood, we can assume that $\rho_{i}^{-1}\left(C^{\prime}\right)$ contains $b_{i}$.

Lemma 6.15 By enlarging $C^{\prime}$ further within $\left(M_{\Gamma}\right)_{0}$ preserving its adequacy, we can make $\rho_{i}^{-1}\left(C^{\prime}\right)$ also an adequate compact core for any large $i$ (with respect to the covering $p_{i}: \widetilde{M}_{i} \rightarrow M_{i}$ ).

Proof Since $C^{\prime}$ is adequate, we can assume that $\rho_{i}^{-1}\left(C^{\prime}\right)$ contains the branching locus of the covering $p_{i}$ for every $i$ by taking a subsequence. What remains to show is that the complement of $\rho_{i}^{-1}\left(C^{\prime}\right)$ has a product structure. 
Fix some simple closed curve $d$ contained in $\mathcal{D}(C)$. We assume that $d$ is not contained in $C^{1}$. Since the bending lamination, which we denote by $\beta_{i}$, of the convex core $C\left(M_{i}\right)$ of $M_{i}$ is contained in $\mathcal{D}(C)$ and $\mathcal{D}(C)$ is arcwise connected [28, Proposition 4.2 ], we can connect $d$ and $\beta_{i}$ by an arc in $\mathcal{D}(C)$. This gives rise to a continuous family of pleated surfaces and negatively curved pleated surfaces in $M_{i}$ realising a homotopy $H_{i}$ between a pleated surface realising $d$ and the boundary of the convex core $C\left(M_{i}\right)$. (This fact can be shown by the argument in Section 4.E of [49], simply replacing the Masur domain by $\mathcal{D}$ since it was shown that every lamination in $\mathcal{D}$ is realised in $M_{i}$ by Theorem 5.1 in [28].) Since pleated surfaces realising $d$ in the $M_{i}$ converge geometrically to one in $M_{\Gamma}$ realising $d$, there is a uniform bound for both their diameters and the distances from them to the $\rho_{i}^{-1}\left(C^{\prime}\right)$. By enlarging $C^{\prime}$, we can assume that $\rho_{i}^{-1}\left(C^{\prime}\right)$ contains the pleated surface realising $d$ for every $i$.

For any sufficiently large $i$, the homotopy $H_{i}$ contains a subhomotopy $H_{i}^{\prime}$ between a pleated surface touching the boundary of $\rho_{i}^{-1}\left(C^{\prime}\right)$ and the boundary of the convex core of $M_{i}$, whose image is disjoint from the bouquet $b_{i}$. We can see as follows that there is an upper bound independent of $i$ for the diameters modulo the $\epsilon$-Margulis tubes in $M_{i}$ of the pleated surfaces (and negatively curved pleated surfaces) constituting $H_{i}^{\prime}$. Suppose, seeking a contradiction, that such an upper bound does not exist. Then, there exist a (negatively curved) pleated surface $f_{i}$ constituting $H_{i}^{\prime}$ and a sequence of simple closed curves $\delta_{i}$ on $\partial C$ such that length $\left(f_{i}\left(\delta_{i}\right)\right) \rightarrow 0$ and $f_{i}\left(\delta_{i}\right)$ is not contained in an $\epsilon-$ Margulis tube. Then $f_{i}\left(\delta_{i}\right)$ must be null-homotopic. Since $f_{i}$ is homotopic to $\partial C\left(M_{i}\right)$ outside $b_{i}$, and any compressing disc of $C\left(M_{i}\right)$ intersects $b_{i}$, we see that $f_{i}\left(\delta_{i}\right)$ bounds a singular disc with area going to 0 which intersects $b_{i}$ essentially. By taking an annular neighbourhood of $\delta_{i}$ on $\partial C$ with height going to $\infty$, consisting of circles of small lengths all of which bound singular discs with area going to 0 , we see that the length of some arc $b_{i}$ has length going to $\infty$ as $i \rightarrow \infty$. (Refer to the proof of Lemma 6.2 in [49] for a similar argument.) This is a contradiction.

In a similar way, we can show that the distance between $b_{i}$ and $\partial C\left(M_{i}\right)$ goes to $\infty$ as follows. We refer the reader to the proof of Theorem 2.1 in [46] for a detailed account of the argument which we shall give briefly below. Suppose, seeking a contradiction, that the distance is bounded after taking a subsequence. There is a positive lower bound for the lengths of compressing curves on $\partial C\left(M_{i}\right)$ since any compressing disc must intersect $b_{i}$ essentially, and we can argue as in the previous paragraph. Since the total length of $b_{i}$ is bounded as $i \rightarrow \infty$, if the distance between $b_{i}$ and $\partial C\left(M_{i}\right)$ is bounded, so is the distance from $x_{i}$ to $\partial C\left(M_{i}\right)$. Therefore, choosing a basepoint within a bounded distance from $x_{i}$, the surface $\partial C\left(M_{i}\right)$ converges geometrically to a pleated surface in $M_{\Gamma}$ as $i \rightarrow \infty$. Although the limit surface may not be embedded, it can be approximated by an embedded surface arbitrarily closely because the $\partial C\left(M_{i}\right)$ 
are embedded. The approximating surfaces are separating in $\left(M_{\Gamma}\right)_{0}$ since $\partial C\left(M_{i}\right)$ is separating. Recall that by Lemma $6.11,\left(M_{\Gamma}\right)_{0}$ has only one end, which is geometrically infinite. Therefore, there are closed geodesics in a noncompact component of the complement of an embedded surface approximating the limit surface, arbitrarily far from the limit surface. Take such a closed geodesic $c_{\infty}$ sufficiently far from the limit surface. We can pull back $c_{\infty}$ to $M_{i}$ by $\rho_{i}^{-1}$ for large $i$ as a quasigeodesic converging to $c_{\infty}$ geometrically. It follows that the closed geodesic homotopic to $\rho_{i}^{-1}\left(c_{\infty}\right)$ is also far from $\partial C\left(M_{i}\right)$, and is not contained in $C\left(M_{i}\right)$. This contradicts the fact that every closed geodesic lies in $C\left(M_{i}\right)$.

Since $\partial C\left(M_{i}\right)$ is incompressible in $M_{i} \backslash b_{i}$, so is every surface constituting $H_{i}^{\prime}$. By the result of Freedman, Hass and Scott [22], we can homotope in $M_{i} \backslash b_{i}$ each surface to an embedding preserving the upper bound of the diameter of the surface modulo the $\epsilon$-Margulis tubes. Therefore, we see that $H_{i}^{\prime}$ gives rise to a product structure $\partial C \times[0,1]$ starting from a surface within a uniformly bounded distance from $\rho_{i}^{-1}\left(C^{\prime}\right)$ and ending at $\partial C\left(M_{i}\right)$ such that $\partial C \times\{t\}$ is incompressible in $M_{i} \backslash b_{i}$ and has diameter modulo the $\epsilon$-Margulis tubes uniformly bounded from above. To be more precise, we can homotope $H_{i}^{\prime}$ in the complement of $b_{i}$ to an embedding $H_{i}^{\prime \prime}: \partial C \times[0,1] \rightarrow C\left(M_{i}\right)$ such that $H_{i}^{\prime \prime}(\partial C \times\{0\})$ lies within the $R$-neighbourhood of $\rho_{i}^{-1}\left(C^{\prime}\right), H_{i}^{\prime \prime}(\partial C \times\{1\})=\partial C\left(M_{i}\right)$, and the diameter of $H_{i}^{\prime \prime}(\partial C \times\{t\})$ modulo the $\epsilon$-Margulis tubes is bounded by a constant independent of $t$ and $i$. We can fix a positive number $K$ so that for every large $i$ there exists a surface $F_{i}(x)=H_{i}^{\prime \prime}\left(x, t_{i}\right)$ with $x \in \partial C$ such that $F_{i}(\partial C)$ intersects the $K$-neighbourhood of $\rho_{i}^{-1}\left(C^{\prime}\right)$ whereas $H_{i}^{\prime}\left(\partial C,\left[t_{i}, 1\right]\right)$ is disjoint from the $K / 2$-neighbourhood of $\rho_{i}^{-1}\left(C^{\prime}\right)$. (To show such $K$ exists, we use the facts that the diameters modulo the $\epsilon$-Margulis tubes of surfaces constituting $H_{i}^{\prime}$ are uniformly bounded, which was proved above and that the distance from $x_{i}$ to $\partial C_{i}$ goes to $\infty$.) Then for large $i$, its pushforward $\rho_{i} \circ F_{i}$ is contained in $\partial C^{\prime} \times(0, \infty)$.

If $\rho_{i} \circ F_{i}$ is compressible in $\partial C^{\prime} \times(0, \infty)$, it can be compressed in an arbitrary $\partial C^{\prime} \times[a, b]$ containing the image of $\rho_{i} \circ F_{i}$; hence $F_{i}$ must be also compressible in $M_{i} \backslash b_{i}$, which is a contradiction. Therefore, $\rho_{i} \circ F_{i}$ is incompressible in $\partial C^{\prime} \times(0, \infty)$. Since the diameters of the $\rho_{i} \circ F_{i}$ modulo the $\epsilon$-thin part are bounded above and $F_{i}(\partial C)$ intersects the $K$-neighbourhood of $\rho_{i}^{-1}\left(C^{\prime}\right)$, there exists $L>0$ such that $\partial C^{\prime} \times(0, L)$ contains all the $\rho_{i} \circ F_{i}(\partial C)$. Since $F_{i}(\partial C)$ is homotopic to $H_{i}^{\prime \prime}(\partial C, t)$ in the complement of $\rho_{i}^{-1}\left(C^{\prime}\right)$, the product structure induced from $\rho_{i}^{-1}\left(\partial C^{\prime} \times(0, L)\right)$ and that of $\partial C \times(0,1)$ are isotopic outside $\rho^{-1}\left(C^{\prime}\right)$. This gives rise to a product structure between $\partial \rho_{i}^{-1}\left(C^{\prime}\right)$ and the boundary of $C\left(M_{i}\right)$.

On the other hand, since $M_{i}$ is convex cocompact (note that $M_{i}$ does not have parabolic elements in this case (2)), $M_{i} \backslash C\left(M_{i}\right)$ has a product structure homeomorphic to $\partial C \times \mathbb{R}$, 
where $\partial C\left(M_{i}\right) \times\{-\infty\}$ is identified with $\partial C\left(M_{i}\right)$. By pasting these product structures, we get a product structure of the complement of $\rho_{i}^{-1}\left(C^{\prime}\right)$. Therefore $\rho_{i}^{-1}\left(C^{\prime}\right)$ is an adequate compact core for large $i$.

We isotope $\Phi$ so that $\Phi(C)=C^{\prime}$, where $C^{\prime}$ is enlarged as above.

Let $g_{i}:\left(\partial C, m_{i}\right) \rightarrow M_{i}$ be a pleated surface homotopic to $\Phi_{i} \mid \partial C$ realising $\mu \cup C^{1}$.

Lemma 6.16 The diameters of the pleated surfaces $g_{i}$ modulo the $\epsilon$-Margulis tubes are bounded above by a constant depending only on $\epsilon$. Furthermore the distance between $\rho_{i}^{-1}\left(C^{\prime}\right)$ and $g_{i}(\partial C)$ goes to infinity as $i \rightarrow \infty$.

Proof Suppose, seeking a contradiction, that the diameters of the $g_{i}$ modulo the $\epsilon-$ Margulis tubes are not uniformly bounded. Then, by the same argument as before, there is a simple closed curve $d_{i}$ on $\partial C \backslash C^{1}$ whose length with respect to $m_{i}$ goes to 0 such that $g_{i}\left(d_{i}\right)$ is null-homotopic. Since $\mu$ is contained in the Masur domain of $\Sigma=\partial C \backslash C^{1}$, it must intersect a measured lamination $\lambda$ to whose projective class $\left[d_{i}\right]$ converges in $\mathbb{P} \mathcal{M L}(\Sigma)$. Since the length of $d_{i}$ with respect to $m_{i}$ goes to 0 , the same argument as in Lemma 6.2 implies that the length of $\mu$ with respect to $m_{i}$ goes to infinity. On the other hand, the length of $\mu$ with respect to $v_{i}$, which was defined in Definition 3.2, is bounded. Recall that every meridian on $\partial C$ intersects $\mu \cup C^{1}$; hence its length with respect to $v_{i}$ goes to $\infty$. Then by the result of Canary [15], the length of $g_{i}(\mu)$ in $M_{i}$ is bounded as $i \rightarrow \infty$. This is a contradiction, and we have shown the first statement.

We shall next show the second statement of our lemma. As was shown in the proof of Lemma 6.2, the image $g_{i}(\mu)$ cannot go into $\epsilon_{i}$-Margulis tubes with $\epsilon_{i} \rightarrow 0$. If the image of $g_{i}$ stays within bounded distance from $\rho_{i}^{-1}\left(C^{\prime}\right)$, it converges geometrically to a pleated surface realising $\mu$ in $M_{\Gamma}$ since $\left\{\phi_{i}(G)\right\}$ converges to $\Gamma$ strongly. This contradicts Lemma 6.2. Therefore the distance between $\rho_{i}^{-1}\left(C^{\prime}\right)$ and $g_{i}(\partial C)$ goes to infinity as $i \rightarrow \infty$.

This lemma implies in particular that for sufficiently large $i$, the image of $g_{i}$ is contained in the complement $U_{i}$ of $\rho_{i}^{-1}\left(C^{\prime}\right)$, which has a product structure homeomorphic to $\partial C \times(0, \infty)$ by Lemma 6.15 .

Lemma 6.17 The pleated surface $g_{i}$ is incompressible outside $\rho_{i}^{-1}\left(C^{\prime}\right)$.

Proof Suppose that $g_{i}$ is compressible in the complement of $\rho_{i}^{-1}\left(C^{\prime}\right)$, which has a product structure by Lemma 6.15 as $U_{i} \cong \partial C \times(0, \infty) \subset M_{i}$. Then we can extend $g_{i}$ 
to a map from a compression body whose exterior boundary is identified with $\partial C$, as can be seen by homotoping the map into $\partial C \times\{\mathrm{pt}\}$ and using the simple loop conjecture proved by Gabai [23]. Since $\partial C^{\prime} \times(0, \infty)$ does not contain an immersed incompressible surface with genus less than that of $\partial C^{\prime} \cong \partial C$, this compression body must be a handlebody, hence can be identified with $C$. (Since $g_{i}$ is homotopic to $\Phi_{i} \mid C$, any compression of $g_{i}$ can be done within $C$.) Since the compression can be performed in any $\partial C^{\prime} \times[a, b]$ containing the image of $g_{i}$, there is an extension $\widehat{g}_{i}: C \rightarrow M_{i}$ of $g_{i}$ whose image has distance from $\rho_{i}^{-1}\left(C^{\prime}\right)$ going to $\infty$. Let $\sigma$ be a spine of $C$, ie a one-complex which is a deformation retract of $C$. Note that there is a retraction $r: C \rightarrow \sigma$ such that for each nonvertex point $x$ in $\sigma$, its preimage $r^{-1}(x)$ is a meridian of $C$. By Lemma 6.15 , we can consider the projection $\pi_{i}$ of $\bar{U}_{i} \cong \partial C^{\prime} \times[0, \infty)$ to $\partial \rho_{i}^{-1}\left(C^{\prime}\right)$. We also consider the projection of $\mu \cup C^{1}$ under $r$. The transverse measure of $\mu \cup C^{1}$ defines a weight for each point of $\sigma$. Since each nonvertex point of $\sigma$ corresponds to a meridian on $\partial C$ and $\mu \cup C^{1}$ is contained in $\mathcal{D}(C)$, the image $r\left(\mu \cup C^{1}\right)$ passes through every point of $\sigma$ with weight bounded below by a positive constant $\eta$. Therefore, if $\pi_{i} \circ \widehat{g}_{i}(\sigma)$ has an essential self-intersection, then $i\left(\left(\pi_{i} \circ g_{i}\right)_{*}\left(\mu \cup C^{1}\right),\left(\pi_{i} \circ g_{i}\right)_{*}\left(\mu \cup C^{1}\right)\right) \geq \eta^{2}$. See Figure 2 .

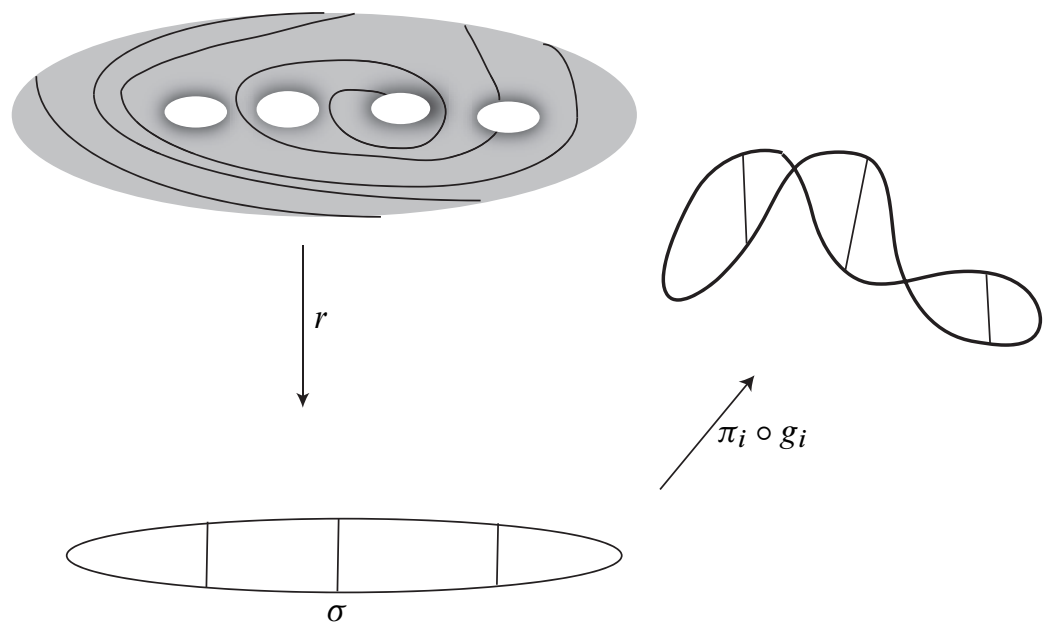

Figure 2: The image of a lamination in $\mathcal{D}(C)$ passes through every point of $\sigma$. Each self-intersection of $\pi_{i} \circ g_{i}(\sigma)$ contributes $\eta^{2}$ to $i\left(\left(\pi_{i} \circ g_{i}\right)_{*}\left(\mu \cup C^{1}\right),\left(\pi_{i} \circ g_{i}\right)_{*}\left(\mu \cup C^{1}\right)\right)$.

We shall prove that $\pi_{i} \circ \widehat{g}_{i}(\sigma)$ has no self-intersection, hence is embedded. For that, we shall show that the homotopy class of $\rho_{i} \circ \pi_{i} \circ \hat{g}_{i}$ is independent of $i$ for large $i$. We extend the conformal structures $v_{i}$ defined in Definition 3.2 to a continuous family $v_{t}$ 
for $t \in[1, \infty)$ such that $v_{t}$ escapes from any compact set of $\mathcal{T}(\partial C)$ and the length of $\mu \cup C^{1}$ with respect to $v_{t}$ is bounded as $t \rightarrow \infty$. For each $t \in[i, i+1]$ with $i \in \mathbb{N}$, let $\left(G_{t}, \phi_{t}\right) \in A H(G)$ be the convex cocompact Kleinian group corresponding to $q\left(v_{t}\right)$, and set $M_{t}$ to be $M_{G_{t}}$ with homeomorphism $\Phi_{t}: M_{G} \rightarrow M_{t}$ induced from $\phi_{t}$. Now, we shall show the existence of a homeomorphism $\bar{\Phi}_{t}: M_{G} \rightarrow M_{t}$ homotopic to $\Phi_{t}$ which has good properties as follows.

Claim 6.18 For each $t \in[1, \infty)$, there is a homeomorphism $\bar{\Phi}_{t}: M_{G} \rightarrow M_{t}$ homotopic to $\Phi_{t}$ with the following properties.

(i) The diameters of $\bar{\Phi}_{t}(C)$ are bounded by a constant independent of $t$.

(ii) When $t$ is an integer $i$, we have $\bar{\Phi}_{i}\left|C=\rho_{i}^{-1} \circ \Phi\right| C$.

(iii) As $t \rightarrow t_{0}$ in $[1, \infty)$, the homeomorphism $\bar{\Phi}_{t}$ converges to $\bar{\Phi}_{t_{0}}$ geometrically.

Proof We can take $C$ to be an adequate compact core. We fix a homotopy class of simple closed curve $b$ in $\mathcal{M}(\Sigma)$. Then it is obvious that $C^{1} \cup b$ is "disc-busting" in Canary's sense, ie intersects any compressing disc of $C$ essentially. When we consider a branched covering $p_{t}: \widetilde{M}_{t} \rightarrow M_{t}$, its branching locus is always assumed to be the closed geodesic (or its perturbation if the closed geodesic is not simple) homotopic to $\Phi_{t}(b)$. We take branched coverings which vary continuously with $t$ in the geometric topology. Let $D_{t}$ be the infimum of the diameters of all compact cores in $M_{t}$ that are adequate with respect to $p_{t}$. We shall first show that $D_{t}$ is bounded from above by a constant independent of $t$.

Suppose, seeking a contradiction, that $D_{t}$ is not bounded. Let $\left\{t_{j}\right\}$ be any monotone increasing sequence in $[1, \infty)$ such that $D_{t_{j}} \rightarrow \infty$. Then by applying our result for $\left\{\phi_{i}\right\}$ in the previous section, regarding $\phi_{t_{j}}$ as $\phi_{i}$, we see that there is a subsequence $\left\{t_{j}^{\prime}\right\}$ of $\left\{t_{j}\right\}$ such that $\left(G_{t_{j}^{\prime}}, \phi_{t_{j}^{\prime}}\right)$ converges to $\left(\Gamma^{\prime}, \phi^{\prime}\right)$, which is either a convex cocompact group or a geometrically infinite group. If $\Gamma^{\prime}$ is convex cocompact, then $\left\{t_{j}^{\prime}\right\}$ is bounded in $[1, \infty), \Gamma^{\prime}=G_{\lim t_{j}}$, and the convergence is strong. If $\Gamma^{\prime}$ is geometrically infinite, then $t_{j}^{\prime}$ goes to $\infty$, and we can apply what we proved for $\Gamma$ up to now in this section. In particular, Lemma 6.14 implies that the convergence to $\Gamma^{\prime}$ is strong.

Set $M^{\prime}=\mathbb{H}^{3} / \Gamma^{\prime}$. Let $\bar{\rho}_{t_{j}^{\prime}}: B_{r_{i}}\left(M_{t_{j}^{\prime}}, x_{t_{j}^{\prime}}\right) \rightarrow B_{K_{i} r_{i}}\left(M^{\prime}, x_{\infty}^{\prime}\right)$ be an approximate isometry associated to the geometric convergence of $M_{t_{j}^{\prime}}$ to $M^{\prime}$. (We put a bar on $\rho$ to distinguish it from the approximate isometries associated to the convergence of $M_{i}$ to $M_{\Gamma}$.) Take an adequate compact core $(\bar{C}, \bar{P})$ of $\left(M^{\prime}\right)_{0}$. Suppose first that $M^{\prime}$ is convex cocompact. Then $M_{t_{j}}$ is quasi-isometric to $M^{\prime}$ and $\bar{\rho}_{t_{j}^{\prime}}$ can be defined on the entire $M_{t_{j}}$ as a bi-Lipschitz homeomorphism. We consider the branched covering of $M^{\prime}$ branched along the closed geodesic homotopic to $\Phi^{\prime}(b)$ or its perturbation 
as usual. Then $\bar{\rho}_{t_{j}^{\prime}}^{-1}(\bar{C})$ is also an adequate compact core for large $j$ since $\bar{\rho}_{t_{j}^{\prime}}$ is a homeomorphism defined on the entire $M_{t_{j}}$ and branching loci move continuously in the geometric topology. This shows that $\left(M_{t_{j}}\right)_{0}$ has an adequate core with diameter less than $D_{t_{j}}$ for large $j$, contradicting the definition of $D_{t_{j}}$.

We shall next show that $\Phi^{\prime}(b)$ does not represent a parabolic class, whether $\Gamma^{\prime}$ is convex cocompact or geometrically infinite. We can assume that $\Phi^{\prime} \mid C$ is a homeomorphism to $\bar{C}$ since both of them are handlebodies. Suppose, seeking a contradiction, that $\Phi^{\prime}(b)$ represents a parabolic class. Then, we can assume that $\Phi^{\prime}(b) \subset \bar{P}$. Since $C^{1} \cup b$ is discbusting, the complement $\bar{F}$ of $\Phi^{\prime}\left(b \cup C^{1}\right)(\subset \bar{P})$ in $\partial \bar{C}$ is incompressible in $\bar{C}$. Also by applying Lemma 6.11 to $\Gamma^{\prime}$, we see that there is only one end of $\left(M^{\prime}\right)_{0}$. Since this end faces an essential subsurface of $\bar{F}$ or $\bar{F}$ itself, which is the incompressible frontier of $\bar{C}$ in $\left(M^{\prime}\right)_{0}$, by Bonahon's theorem, every unrealisable lamination is homotopic to the ending lamination. Therefore $\Phi^{\prime}(\mu)$ must be homotopic to a measured lamination in $\partial C \backslash\left(C^{1} \cup b\right)$, which represents the ending lamination. By the same argument as in the proof of Lemma 6.12, by comparing the complementary regions of $\mu$ and a measured lamination in $\partial C \backslash\left(C^{1} \cup b\right)$, we see that this is impossible. Thus we have shown that $\Phi^{\prime}(b)$ cannot represent a parabolic class, and also that we can consider the branching covering of $M^{\prime}$ branched along the closed geodesic homotopic to $\Phi^{\prime}(b)$ or its perturbation even when $\Gamma^{\prime}$ is geometrically infinite.

Now, in the case when $\Gamma^{\prime}$ is geometrically infinite, Lemma 6.15 tells us that we can enlarge $\bar{C}$ so that $\bar{\rho}_{t_{j}^{\prime}}^{-1}(\bar{C})$ is an adequate compact core for large $j$. This shows that $M_{t_{j}^{\prime}}$ contains an adequate compact core whose diameter is less than $D_{t_{j}^{\prime}}$ for large $j$, which is a contradiction.

Thus we have proved that $D_{t}$ is uniformly bounded from above, whether $\Gamma^{\prime}$ is geometrically infinite or not. We have also shown above that there is a positive lower bound $\xi$ for the lengths of the closed geodesics homotopic to the $\Phi_{t}(b)$. Let $D$ be a constant which bounds from above both the $D_{t}+1$ for all $t \in[1, \infty)$ and the diameters of $C^{\prime}$ and the $\rho_{i}^{-1}\left(C^{\prime}\right)$.

Next, we shall show that there is a constant $E$ independent of $t$ such that $M_{t}$ has an adequate compact core $\widehat{C}_{t}$ with diameter less than $2 D$ whose $4 D$-neighbourhood can be isotoped into $\widehat{C}_{t}$ passing only the $E$-neighbourhood of $\widehat{C}_{t}$. Suppose, seeking a contradiction, that such $E$ does not exist. Then, there is monotone increasing sequence $\left\{t_{j}\right\}$ in $[1, \infty)$ such that any adequate compact core $\widehat{C}_{t_{j}}$ of $M_{t_{j}}$ with diameter less than $2 D$ has $4 D$-neighbourhood which cannot be isotoped into $\widehat{C}_{t_{j}}$ within the $E_{j}$-neighbourhood of $\widehat{C}_{t_{j}}$, where $E_{j}$ goes to $\infty$ as $j \rightarrow \infty$. As before, after passing to a subsequence, $\left\{\left(G_{t_{j}}, \phi_{t_{j}}\right)\right\}$ converges strongly to $\left(\Gamma^{\prime}, \phi^{\prime}\right)$ which is either convex cocompact or geometrically infinite, and $\left(M^{\prime}\right)_{0}$ has an adequate compact core $\bar{C}$. By 
the same argument as above, we can enlarge $\bar{C}$ so that for sufficiently large $j$, the submanifold $\bar{\rho}_{t_{j}}^{-1}(\bar{C})$ is an adequate compact core of $M_{t_{j}}$. We can choose such an enlarged adequate compact core to have diameter bounded by $D$ by our definition of $D$. Since $\bar{C}$ is an adequate compact core in $\left(M^{\prime}\right)_{0}$, there is a constant $E^{\prime}$ such that the 5D-neighbourhood of $\bar{C}$ can be isotoped into $\bar{C}$ passing only the $E^{\prime}$-neighbourhood of $\bar{C}$. Pulling this back to $M_{t_{j}}$ for large $j$, we see that the $4 D$-neighbourhood of $\bar{\rho}_{t_{j}}^{-1}(\bar{C})$ can be isotoped into $\rho_{t_{j}}^{-1}(\bar{C})$ passing only the $2 E^{\prime}$-neighbourhood of $\bar{\rho}_{t_{j}}^{-1}(\bar{C})$. Since the diameter of $\bar{C}$ is bounded by $D$, we see that the diameter of $\bar{\rho}_{t_{j}}^{-1}(\bar{C})$ is less than $2 D$ for large $j$. Since $E_{j}$ is greater than $2 E^{\prime}$ for large $j$, this is a contradiction. Thus we have proved the existence of $E$ as above.

For $D$ as was given above, we take $K_{0} \in(1,2]$ so that for any $K \leq K_{0}$ and any $K$-bi-Lipschitz homeomorphism $f: N \rightarrow N^{\prime}$ between hyperbolic 3-manifolds, if a closed geodesic $c$ in $N$ has length greater than $\xi$, which was defined to be a lower bound for the lengths of the closed geodesics homotopic to the $\Phi_{t}(b)$, then $f(c)$ is contained in the $D$-neighbourhood of the closed geodesic homotopic to $f(c)$. The existence of such $K_{0}$ follows easily from the well-known properties of quasigeodesics.

Now, for each $i \in \mathbb{N}$, we subdivide $[i, i+1]$ into intervals $i=s_{0}<s_{1}<\cdots<s_{m-1}<$ $s_{m}=i+1$ in such a way that for any $t, t^{\prime} \in\left[s_{j}, s_{j+1}\right]$, the quasiconformal deformation from $\left(G_{t}, \phi_{t}\right)$ to $\left(G_{t^{\prime}}, \phi_{t^{\prime}}\right)$ induces (by extending quasiconformal maps to quasiisometries in $\mathbb{H}^{3}$ in the standard way) a $\left(K_{0}\right)^{1 / 2}$-bi-Lipschitz homeomorphism $\Psi_{t, t^{\prime}}$ from $M_{t}$ to $M_{t^{\prime}}$ which is homotopic to $\Phi_{t^{\prime}} \circ \Phi_{t}^{-1}$. (The number $m$ may depend on $i$.) Since $\left\{\left(G_{t}, \phi_{t}\right)\right\}$ is a family of quasiconformal deformations which is continuous with respect to the parameter $t$, any subdivision whose maximal width is sufficiently small serves as a subdivision as above. Now for each $s_{j}(j \neq 0, m)$, we define $\bar{C}_{s_{j}}$ to be an adequate compact core of $M_{s_{j}}$ whose diameter is less than $D_{s_{j}}+1$, which is guaranteed to exist by our definition of $\underline{D}_{t}$. For $j=0, m$, we define $\bar{C}_{s_{j}}$ to be $\rho_{i}^{-1}\left(C^{\prime}\right)$ and $\rho_{i+1}^{-1}\left(C^{\prime}\right)$ respectively. We define $\bar{\Phi}_{s_{j}} \mid C$ to be a homeomorphism from $C$ to $\bar{C}_{s_{j}}$ homotopic in $M_{s_{j}}$ to $\Phi_{s_{j}} \mid C$. In the case when $j=0, m$ we choose the homeomorphism to be $\rho_{i}^{-1} \circ \Phi$. Since both $C$ and $\bar{C}_{s_{j}}$ are adequate, their complements have product structures and we can extend $\bar{\Phi}_{s_{j}} \mid C$ to a homeomorphism $\bar{\Phi}_{s_{j}}: M_{G} \rightarrow M_{s_{j}}$. Then we see that the diameter of $\bar{\Phi}_{t}(C)$ is bounded by $D$ for $t=s_{0}, \ldots, s_{m}$, and (ii) in our claim holds for $\bar{\Phi}_{t}$ defined thus far (ie when $t$ is a subdividing point).

We need to extend the family of homeomorphisms to all the parameters $t \in[1, \infty)$. Now we further subdivide $\left[s_{j}, s_{j+1}\right]$ into three subintervals of the same length, $\left[s_{j}, s_{j}^{\prime}\right],\left[s_{j}^{\prime}, s_{j}^{\prime \prime}\right]$ and $\left[s_{j}^{\prime \prime}, s_{j+1}\right]$. We consider a $\left(K_{0}\right)^{1 / 2}$-bi-Lipschitz homeomorphism $\Psi_{s_{j}, t}$ for $t \in$ $\left[s_{j-1}^{\prime}, s_{j}^{\prime}\right]$. Since this bi-Lipschitz homeomorphism $\Psi_{s_{j}, t}$ is induced from a quasiconformal deformation from $\left(G_{s_{j}}, \phi_{s_{j}}\right)$ to $\left(G_{t}, \phi_{t}\right)$, we can assume that it varies continuously with respect to $t$ in the geometric topology, and $\Psi_{s_{j}, t}$ converges to the 
identity geometrically as $t \rightarrow s_{j}$. For each $t \in\left[s_{j}, s_{j}^{\prime}\right]$, we define $\bar{\Phi}_{t}$ to be $\Psi_{s_{j}, t} \circ \bar{\Phi}_{s_{j}}$, and for each $t \in\left[s_{j}^{\prime \prime}, s_{j+1}\right]$, we define $\bar{\Phi}_{t}$ to be $\Psi_{s_{j+1}, t} \circ \bar{\Phi}_{s_{j+1}}$. Now, we shall fill the gap between $\bar{\Phi}_{s_{j}^{\prime}}$ and $\bar{\Phi}_{s_{j}^{\prime \prime}}$.

Since both $\bar{C}_{s_{j}}$ and $\bar{C}_{s_{j+1}}$ have diameters bounded by $D$, the compact cores $\bar{\Phi}_{s_{j}^{\prime}}(C)$ and $\Psi_{s_{j+1}, s_{j}^{\prime}} \circ \bar{\Phi}_{s_{j+1}}(C)$ have diameters bounded by $2 D$ (because $K_{0}^{1 / 2} \leq \sqrt{2}^{2}<2$ ). Moreover, since $\bar{C}_{s_{j}}$ contains the closed geodesic $b_{s_{j}}^{*}$ homotopic to $\Phi_{s_{j}}(b)$, and $\Psi_{s_{j}, s_{j}^{\prime}}$ is $\left(K_{0}^{1 / 2}-\right.$, hence also) $K_{0}$-bi-Lipschitz, $\bar{\Phi}_{s_{j}^{\prime}}(C)$, which contains $\Psi_{s_{j}, s_{j}^{\prime}}\left(b_{s_{j}}^{*}\right)$, is within distance $D$ from any point on the closed geodesic homotopic to $\Phi_{s_{j}^{\prime}}(b)$. By considering the same for $\Psi_{s_{j+1}, s_{j}^{\prime}} \circ \bar{\Phi}_{s_{j+1}}(C)=\Psi_{s_{j+1}, s_{j}^{\prime}}\left(\bar{C}_{s_{j+1}}\right)$, we see that $\Psi_{s_{j+1}, s_{j}^{\prime}} \circ \bar{\Phi}_{s_{j+1}}(C)$ is within distance $2 D$ from $\bar{\Phi}_{s_{j}^{\prime}}(C)$, hence is contained in the $4 D$-neighbourhood of $\bar{\Phi}_{s_{j}^{\prime}}(C)$. By our definition of $E$, there is an isotopy taking $\Psi_{s_{j+1}, s_{j}^{\prime}} \circ \bar{\Phi}_{s_{j+1}}(C)$ into $\bar{\Phi}_{s_{j}^{\prime}}(C)$ which passes only the $E$-neighbourhood of $\bar{\Phi}_{s_{j}^{\prime}}(C)$. Since the complement of $C$ has a product structure, we can extend this isotopy to an isotopy $H: M_{G} \times\left[s_{j}^{\prime}, s_{j}^{\prime \prime}\right] \rightarrow M_{s_{j}^{\prime}}$ such that each $H(, t)$ is a homeomorphism to $M_{s_{j}^{\prime}}, H\left(, s_{j}^{\prime}\right)=\bar{\Phi}_{s_{j}^{\prime}}, H\left(, s_{j}^{\prime \prime}\right)=\Psi_{s_{j+1}, s_{j}^{\prime}} \circ \bar{\Phi}_{s_{j+1}}$, and $H(C, t)$ is contained in the $E$-neighbourhood of $\bar{\Phi}_{s_{j}^{\prime}}(C)$. Now, for $t \in\left[s_{j}^{\prime}, s_{j}^{\prime \prime}\right]$, define $\bar{\Phi}_{t}$ to be $\Psi_{s_{j+1}, t} \circ \Psi_{s_{j+1}, s_{j}^{\prime}}^{-1} \circ H(, t)$. Because $\Psi_{s_{j+1}, t} \circ \Psi_{s_{j+1}, s_{j}^{\prime}}^{-1}$ is $K_{0}$-bi-Lipschitz with $K_{0} \leq 2$, we see that $\bar{\Phi}_{t}(C)$ has diameter bounded by $2(2 D+E)$. Thus we have completed the definition of $\bar{\Phi}_{t}$, which have the properties (i) and (ii). The continuity with respect to the geometric topology is obvious from our definition of $\bar{\Phi}_{t}$.

Now we return to the proof of Lemma 6.17. Let $g_{t}: \partial C \rightarrow M_{t}$ be a pleated surface homotopic to $\Phi_{t}$ which realises $\mu \cup C^{1}$. Since the length of $\mu$ with respect to $v_{t}$ is bounded as $t \rightarrow \infty$ and $C^{1} \cup \mu$ intersects every compressing curve, by the same argument as for $g_{i}$, we see the length of $g_{t}(\mu)$ is bounded as $t \rightarrow \infty$. Since the diameter of $\bar{\Phi}_{t}(C)$ is bounded as $t \rightarrow \infty$ by Claim 6.18 , by the same argument as for $g_{i}$, it follows that the surface $g_{t}(\partial C)$ is disjoint from $\bar{\Phi}_{t}(C)$ for large $t$. Note that both $g_{t}(\mu)$ and $\bar{\Phi}_{t}$ vary continuously with respect to the geometric topology. Let $U_{t} \cong \partial C \times(0, \infty)$ be a parametrisation of the complement of $\bar{\Phi}_{t}(C)$. Let $\pi_{t}: M_{t} \rightarrow$ $\partial \bar{\Phi}_{t}(C)$ be the projection to the first factor in $\bar{U}_{t} \cong \partial C \times[0, \infty)$, where we identify $\partial C \times\{0\}$ with $\bar{\Phi}_{t}(\partial C)$. Since the parametrisation and the projection are unique up to homotopy, we can assume that $U_{t}$ and $\pi_{t}$ vary continuously with respect to the geometric topology. Since $g_{t}(\partial C)$ never touches $\bar{\Phi}_{t}(C)$, this implies that the homotopy class of $\bar{\Phi}_{t}^{-1} \circ \pi_{t} \circ g_{t}$ is independent of $t$. Recall that $\rho_{i}^{-1}\left|C^{\prime}=\bar{\Phi}_{i} \circ \Phi^{-1}\right| C^{\prime}$ for every $i \in \mathbb{N}$. Therefore for $i \in \mathbb{N}$, we see that the homotopy class of $\rho_{i} \circ \pi_{i} \circ g_{i}$ as a map from $\partial C$ to $\partial C^{\prime}$ is independent of $i$. Let $p: \partial C \rightarrow \partial C^{\prime}$ be a map homotopic to these maps. If $\pi_{i} \circ g_{i}(\sigma)$ has essential self-intersection, then $i\left(p_{*}\left(\mu \cup C^{1}\right), p_{*}\left(\mu \cup C^{1}\right)\right) \geq \eta^{2}$ for a positive constant $\eta$ independent of $i$ as was shown before. 
Take a sequence of weighted simple closed curves $\left\{r_{k} c_{k}\right\}$ on $\Sigma=\partial C \backslash P$ converging to $\mu$. By taking a subsequence of $\left\{r_{k} c_{k}\right\}$, we can assume that for each $i$, there is a pleated surface $g_{i}^{\prime}: \partial C \rightarrow M_{i}$ realising $C^{1} \cup c_{i}$ which is homotopic to $g_{i}$ in $U_{i}$, whose diameter modulo the $\epsilon$-Margulis tubes is bounded as $i \rightarrow \infty$. We can extend $g_{i}^{\prime}$ to $\hat{g}_{i}^{\prime}: C \rightarrow M_{i}$ by the same way as for $g_{i}$. By Lemma 6.10, there is a sequence of weighted simple closed curves $R_{k} C_{k}$ on $\partial C \backslash P$ converging to $\mu^{\prime}$ with $i\left(\mu, \mu^{\prime}\right)=0$ such that $i\left(R_{i} \pi_{i} \circ g_{i}^{\prime}\left(C_{i}\right), R_{i} \pi_{i} \circ g_{i}^{\prime}\left(C_{i}\right)\right) \rightarrow 0$. Since $g_{i}^{\prime}$ is homotopic to $g_{i}$ in $U_{i}$, we get $i\left(R_{i} p\left(C_{i}\right), R_{i} p\left(C_{i}\right)\right) \rightarrow 0$, which implies $i\left(p_{*}\left(\mu^{\prime}\right), p_{*}\left(\mu^{\prime}\right)\right)=0$, hence also $i\left(p_{*}(\mu), p_{*}(\mu)\right)=0$. Since $\pi_{i} \circ g_{i}^{\prime}\left(C_{i}\right)$ is disjoint from $\Phi_{i}\left(C^{1}\right)$ as was shown in Lemma 6.10, we have $i\left(p_{*}\left(\mu^{\prime}\right), p\left(C^{1}\right)\right)=0$. These show that $i\left(p_{*}\left(\mu \cup C^{1}\right), p_{*}\left(\mu \cup C^{1}\right)\right)=0$. Thus $\sigma$ can be embedded on $\partial C^{\prime}$ by $p$ if we move $p$ by a homotopy on $\partial C^{\prime}$.

We have also shown that $p_{*}(\mu)$, regarded as a geodesic current, is actually a measured lamination on $\partial C^{\prime} \backslash P^{\prime}$ since it has null self-intersection. On the other hand since $p \mid \sigma$ is embedding, $p_{*}(\mu)$ has a large complementary region, which is contained in $\partial C^{\prime} \backslash\left(P^{\prime} \cup p(\sigma)\right)$. Then by the same argument as in the proof of Lemma 6.12 considering the correspondence between the complementary regions of $p_{*}(\mu)$ and a measured lamination representing the ending lamination for the unique end of $\left(M_{\Gamma}\right)_{0}$ gives a contradiction. So we have shown $g_{i}$ is incompressible in $U_{i} \cong \partial C \times(0, \infty)$.

Since $g_{i}$ is incompressible in $U_{i} \cong \partial C \times(0, \infty)$, we see that $\pi_{i} \circ g_{i}$ is homotopic a homeomorphism from $\partial C$ to $\partial \bar{C}_{i}=\rho_{i}^{-1}\left(\partial C^{\prime}\right)$. As was shown above, the maps $\rho_{i} \circ \pi_{i} \circ g_{i}$ are all homotopic, which we denote by $h: \partial C \rightarrow \partial C^{\prime}$. Note that $h$ is homotopic in $C^{\prime}$ to $\Phi \mid \partial C$, hence can be extended to a homeomorphism from $C$ to $C^{\prime}$. Also since $g_{i}$ maps $C^{1}$ into Margulis tubes converging geometrically to cusps in $M_{\Gamma}$, we can assume that $h(P)=P^{\prime}$. Take a weighted simple closed curve $\left\{s_{k} d_{k}\right\}$ on $\partial C^{\prime} \backslash P^{\prime}$ converging to a measured lamination $\lambda$ representing the ending lamination of the unique end of $\left(M_{\Gamma}\right)_{0}$ such that the closed geodesic $d_{k}^{*}$ homotopic to $d_{k}$ in $U \cong\left(\partial C^{\prime} \backslash P^{\prime}\right) \times[0, \infty)$ tends to the end. Recall that we have a sequence of weighted simple closed curves $\left\{r_{k} c_{k}\right\}$ converging to $\mu$ with a pleated surface $g_{i}^{\prime}$ realising $c_{i} \cup C^{1}$ in $M_{i}$. Then by Lemma 6.10, we have sequences of simple closed curves $C_{k}$ on $\partial C \backslash P$ and $D_{k}$ on $\partial C^{\prime} \backslash P^{\prime}$ such that $\left[C_{k}\right]$ converges to $\left[\mu^{\prime}\right]$ with $i\left(\mu, \mu^{\prime}\right)=0$ and $\left[D_{k}\right]$ converges to $\left[\lambda^{\prime}\right]$ with $i\left(\lambda, \lambda^{\prime}\right)=0$, and we have

$$
i\left(\frac{\pi_{i} \circ g_{i}^{\prime}\left(C_{i}\right)}{\text { length }\left(\pi_{i} \circ g_{i}^{\prime}\left(C_{i}\right)\right)}, \frac{\rho_{i}^{-1}\left(D_{i}\right)}{\text { length }\left(\rho_{i}^{-1}\left(D_{i}\right)\right)}\right) \rightarrow 0 .
$$

By pushing this forward by $\rho_{i}$, we get

$$
i\left(\frac{h\left(C_{i}\right)}{\text { length }\left(h\left(C_{i}\right)\right)}, \frac{D_{i}}{\text { length }\left(D_{i}\right)}\right) \rightarrow 0,
$$


hence $i\left(h\left(\mu^{\prime}\right), \lambda^{\prime}\right)=0$. Since both $\mu$ and $\lambda$ are arational, $\mu^{\prime}$ has the same support as $\mu$, and $\lambda^{\prime}$ has the same one as $\lambda$. It follows that $h(\mu)$ has the same support as $\lambda$. Since $h$ extends to a homeomorphism from $C$ to $C^{\prime}$ taking $P$ to $P^{\prime}$ as was shown above, we have completed the proof of Proposition 6.5 in case (2). Combining Lemma 6.9 with this, we have completed the proof of Proposition 6.5.

\section{Proof of the main theorem}

We shall complete the proof of Theorem 1.2 in this section. By Proposition 6.5, we have shown that $\Phi$ can be homotoped so that $\Phi \mid P$ is an embedding into the parabolic locus $P^{\prime}$ on $\partial C^{\prime}$ and $\Phi \mid \Sigma_{j}$ is a homeomorphism to a component of $\partial C^{\prime} \backslash P^{\prime}$ for $j=n+1, \ldots, m$ such that $\Phi \mid \mu_{j}$ represents the ending lamination for the end facing the component, which is topologically tame. It remains to deal with $\Phi \mid \Sigma_{j}$ for $j=1, \ldots, n$. Let $\Sigma$ be one of the $\Sigma_{j}$ for $j=1, \ldots, n$. Let $S_{k}$ be the boundary component of $C$ containing $\Sigma$, and $H$ a subgroup of $G$ corresponding to the image of $\pi_{1}\left(S_{k}\right)$. Let $H^{\Sigma}$ denote a subgroup of $H$ corresponding to the image of $\pi_{1}(\Sigma)$ in $\pi_{1}(C) \cong G$. Then by the same argument as the proof of Lemma 6.6 using the result of Abikoff, we see that there are an open subset $\Omega^{\prime}$ of $\Omega_{\Gamma}$ invariant under $\phi\left(H^{\Sigma}\right)$, and a frontier component of the convex core of $\left(M_{\Gamma}\right)_{0}$ facing $\Omega^{\prime} / \phi\left(H^{\Sigma}\right)$. Since $C^{\prime}$ is a nice compact core, there is a component of $\partial C^{\prime} \backslash P^{\prime}$ ambient isotopic to this frontier component. Therefore the surface $\Phi \mid \Sigma$ is homotoped to this component of $\partial C^{\prime} \backslash P^{\prime}$ keeping $\Phi($ Fr $\Sigma)$ in $P^{\prime}$. Repeating this argument for every one of the $\Sigma_{j}(j=1, \ldots, n)$, and combining it with Proposition 6.5, we see that $\Phi$ is homotopic to a homeomorphism from $C$ to $C^{\prime}$, hence from $M_{G}$ to $M_{\Gamma}$, by Waldhausen's theorem [59]. Moreover, the ends of $\left(M_{\Gamma}\right)_{0}$ facing these components $\Sigma_{1}, \ldots, \Sigma_{n}$ are geometrically finite and have conformal structure at infinity $m_{1}, \ldots, m_{n}$. This completes the proof of Theorem 1.2.

\section{References}

[1] W Abikoff, On boundaries of Teichmüller spaces and on Kleinian groups. III, Acta Math. 134 (1975) 211-237 MR0435452

[2] I Agol, Tameness of hyperbolic 3-manifolds arXiv:math/0405568

[3] L Bers, On boundaries of Teichmüller spaces and on Kleinian groups. I, Ann. of Math. (2) 91 (1970) 570-600 MR0297992

[4] L Bers, An inequality for Riemann surfaces, from: "Differential geometry and complex analysis", (I Chavel, H M Farkas, editors), Springer, Berlin (1985) 87-93 MR780038

[5] M Bestvina, Degenerations of the hyperbolic space, Duke Math. J. 56 (1988) 143-161 MR932860 
[6] F Bonahon, Cobordism of automorphisms of surfaces, Ann. Sci. École Norm. Sup. (4) 16 (1983) 237-270 MR732345

[7] F Bonahon, Bouts des variétés hyperboliques de dimension 3, Ann. of Math. (2) 124 (1986) 71-158 MR847953

[8] F Bonahon, The geometry of Teichmüller space via geodesic currents, Invent. Math. 92 (1988) 139-162 MR931208

[9] J F Brock, K W Bromberg, On the density of geometrically finite Kleinian groups, Acta Math. 192 (2004) 33-93 MR2079598

[10] J F Brock, R D Canary, Y N Minsky, The classification of Kleinian surface groups, II: The Ending Lamination Conjecture arXiv:math.GT/0412006

[11] J F Brock, J Souto, Algebraic limits of geometrically finite manifolds are tame, Geom. Funct. Anal. 16 (2006) 1-39 MR2221251

[12] K W Bromberg, Projective structures with degenerate holonomy and the Bers density conjecture, Ann. of Math. (2) 166 (2007) 77-93 MR2342691

[13] R Brooks, J P Matelski, Collars in Kleinian groups, Duke Math. J. 49 (1982) 163-182 MR650375

[14] D Calegari, D Gabai, Shrinkwrapping and the taming of hyperbolic 3-manifolds, J. Amer. Math. Soc. 19 (2006) 385-446 MR2188131

[15] R D Canary, The Poincaré metric and a conformal version of a theorem of Thurston, Duke Math. J. 64 (1991) 349-359 MR1136380

[16] R D Canary, Ends of hyperbolic 3-manifolds, J. Amer. Math. Soc. 6 (1993) 1-35 MR1166330

[17] R D Canary, A covering theorem for hyperbolic 3-manifolds and its applications, Topology 35 (1996) 751-778 MR1396777

[18] R D Canary, The conformal boundary and the boundary of the convex core, Duke Math. J. 106 (2001) 193-207 MR1810370

[19] R D Canary, D B A Epstein, P Green, Notes on notes of Thurston, from: "Analytical and geometric aspects of hyperbolic space (Coventry/Durham, 1984)", (D B A Epstein, editor), London Math. Soc. Lecture Note Ser. 111, Cambridge Univ. Press (1987) 3-92 MR903850

[20] D B A Epstein, A Marden, Convex hulls in hyperbolic space, a theorem of Sullivan, and measured pleated surfaces, from: "Analytical and geometric aspects of hyperbolic space (Coventry/Durham, 1984)", (D B A Epstein, editor), London Math. Soc. Lecture Note Ser. 111, Cambridge Univ. Press (1987) 113-253 MR903852

[21] A Fathi, F Laudenbach, V Poenaru, editors, Travaux de Thurston sur les surfaces, Astérisque 66-67, Soc. Math. France (1979) MR568308 Séminaire Orsay, With an English summary 
[22] M Freedman, J Hass, P Scott, Least area incompressible surfaces in 3-manifolds, Invent. Math. 71 (1983) 609-642 MR695910

[23] D Gabai, The simple loop conjecture, J. Differential Geom. 21 (1985) 143-149 MR806708

[24] W H Jaco, P B Shalen, Seifert fibered spaces in 3-manifolds, Mem. Amer. Math. Soc. 21 (1979) viii+192 MR539411

[25] K Johannson, Homotopy equivalences of 3-manifolds with boundaries, Lecture Notes in Math. 761, Springer, Berlin (1979) MR551744

[26] I Kim, C Lecuire, K Ohshika, Convergence of freely decomposable Kleinian groups arXiv:0708.3266

[27] G Kleineidam, J Souto, Algebraic convergence of function groups, Comment. Math. Helv. 77 (2002) 244-269 MR1915041

[28] C Lecuire, An extension of the Masur domain, from: "Spaces of Kleinian groups", (Y N Minsky, M Sakuma, C Series, editors), London Math. Soc. Lecture Note Ser. 329, Cambridge Univ. Press (2006) 49-73 MR2258744

[29] C Lecuire, Plissage des variétés hyperboliques de dimension 3, Invent. Math. 164 (2006) 85-141 MR2207784

[30] B Maskit, Parabolic elements in Kleinian groups, Ann. of Math. (2) 117 (1983) 659-668 MR701259

[31] H A Masur, Y N Minsky, Geometry of the complex of curves. I. Hyperbolicity, Invent. Math. 138 (1999) 103-149 MR1714338

[32] H A Masur, Y N Minsky, Geometry of the complex of curves. II. Hierarchical structure, Geom. Funct. Anal. 10 (2000) 902-974 MR1791145

[33] D McCullough, Compact submanifolds of 3-manifolds with boundary, Quart. J. Math. Oxford Ser. (2) 37 (1986) 299-307 MR854628

[34] Y N Minsky, Teichmüller geodesics and ends of hyperbolic 3-manifolds, Topology 32 (1993) 625-647 MR1231968

[35] Y N Minsky, On rigidity, limit sets, and end invariants of hyperbolic 3-manifolds, J. Amer. Math. Soc. 7 (1994) 539-588 MR1257060

[36] Y N Minsky, The classification of punctured-torus groups, Ann. of Math. (2) 149 (1999) 559-626 MR1689341

[37] Y N Minsky, Kleinian groups and the complex of curves, Geom. Topol. 4 (2000) 117-148 MR2182094

[38] Y N Minsky, The classification of Kleinian surface groups. I. Models and bounds, Ann. of Math. (2) 171 (2010) 1-107 MR2630036 
[39] J W Morgan, On Thurston's uniformization theorem for three-dimensional manifolds, from: "The Smith conjecture (New York, 1979)", (J W Morgan, H Bass, editors), Pure Appl. Math. 112, Academic Press, Orlando, FL (1984) 37-125 MR758464

[40] J W Morgan, J-P Otal, Relative growth rates of closed geodesics on a surface under varying hyperbolic structures, Comment. Math. Helv. 68 (1993) 171-208 MR1214228

[41] J W Morgan, P B Shalen, Valuations, trees, and degenerations of hyperbolic structures. I, Ann. of Math. (2) 120 (1984) 401-476 MR769158

[42] J W Morgan, P B Shalen, Degenerations of hyperbolic structures. II. Measured laminations in 3-manifolds, Ann. of Math. (2) 127 (1988) 403-456 MR932305

[43] H Namazi, J Souto, Non-realizability, ending laminations and the density conjecture, preprint (2010) Available at http://www-personal.umich.edu/ jsouto/ ending6.6.pdf

[44] K Ohshika, Ending laminations and boundaries for deformation spaces of Kleinian groups, J. London Math. Soc. (2) 42 (1990) 111-121 MR1078179

[45] K Ohshika, Limits of geometrically tame Kleinian groups, Invent. Math. 99 (1990) 185-203 MR1029395

[46] K Ohshika, Geometric behaviour of Kleinian groups on boundaries for deformation spaces, Quart. J. Math. Oxford Ser. (2) 43 (1992) 97-111 MR1150472

[47] K Ohshika, Geometrically finite Kleinian groups and parabolic elements, Proc. Edinburgh Math. Soc. (2) 41 (1998) 141-159 MR1604337

[48] K Ohshika, Rigidity and topological conjugates of topologically tame Kleinian groups, Trans. Amer. Math. Soc. 350 (1998) 3989-4022 MR1451613

[49] K Ohshika, Kleinian groups which are limits of geometrically finite groups, Mem. Amer. Math. Soc. 177 (2005) xii+116 MR2154090

[50] K Ohshika, Constructing geometrically infinite groups on boundaries of deformation spaces, J. Math. Soc. Japan 61 (2009) 1261-1291 MR2588511

[51] K Ohshika, H Miyachi, On topologically tame Kleinian groups with bounded geometry, from: "Spaces of Kleinian groups", (Y N Minsky, M Sakuma, C Series, editors), London Math. Soc. Lecture Note Ser. 329, Cambridge Univ. Press (2006) 29-48 MR2258743

[52] J-P Otal, Courants géodésiques et produits libres, Thèse d'Etat, Université Paris-Sud, Orsay (1988)

[53] J-P Otal, Le théorème d'hyperbolisation pour les variétés fibrées de dimension 3, Astérisque 235, Soc. Math. France (1996) MR1402300

[54] F Paulin, Topologie de Gromov équivariante, structures hyperboliques et arbres réels, Invent. Math. 94 (1988) 53-80 MR958589 
[55] G P Scott, Compact submanifolds of 3-manifolds, J. London Math. Soc. (2) 7 (1973) 246-250 MR0326737

[56] R K Skora, Splittings of surfaces, J. Amer. Math. Soc. 9 (1996) 605-616 MR1339846

[57] T Sugawa, Uniform perfectness of the limit sets of Kleinian groups, Trans. Amer. Math. Soc. 353 (2001) 3603-3615 MR1837250

[58] W P Thurston, The geometry and topology of three-manifolds, Princeton Univ. Math. Dept. Lecture Notes (1979) Available at http://msri.org/publications/books/ gt $3 \mathrm{~m} /$

[59] F Waldhausen, On irreducible 3-manifolds which are sufficiently large, Ann. of Math. (2) 87 (1968) 56-88 MR0224099

Department of Mathematics, Graduate School of Science, Osaka University Toyonaka, Osaka 560-0043, Japan

ohshika@math.sci.osaka-u.ac.jp

Proposed: David Gabai

Seconded: Jean-Pierre Otal, Danny Calegari

Received: 15 January 2009

Revised: 11 March 2011 